

\section{EDITOR'S NOTE}

Eureka: A beacon for undergraduate research $\ldots \ldots \ldots \ldots \ldots \ldots \ldots \ldots \ldots \ldots \ldots \ldots \ldots \ldots \ldots \ldots \ldots$ Christopher R. Madan

\section{INTERVIEWS}

The evolving role of the pharmacist and why you should be one $\ldots \ldots \ldots \ldots \ldots \ldots \ldots \ldots \ldots \ldots \ldots$ Pattrick Jones

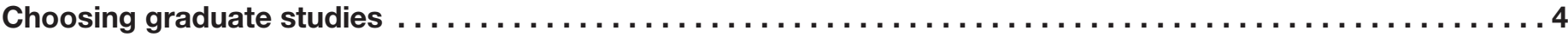

Braden Teitge

How donated blood is tested: An interview with Canadian Blood Services $\ldots \ldots \ldots \ldots \ldots \ldots \ldots \ldots \ldots \ldots \ldots 6$

Eureka Team

\section{PUBLISHED ABSTRACTS}

Biofeedback as a Physiological Treatment for Traumatic Psychological Injuries . . . . . . . . . . . . . 9 Lauren Alston

Self-assessment and observer rating of empathetic communication in pharmacy students. . . . . . . . . Lisa M. Guirguis \& Enoch Ng

\section{ARTICLES}

Sterol Chemical Structure and Conformation Influence the Thermotropic Phase Behaviour of Dipalmitoylphosphatidylcholine Bilayers . . . . . . . . . . . . . . . . . . . . . . . . . . .

Matthew G. K. Benesch, David A. Mannock, \& Ronald N. McElhaney

IR Motion Tracking as a Standard Input Device. . . . . . . . . . . . . . . . . . . . . . . . . . 24

Michael Cimolini

Inability of Viral Superantigens to Induce CD4-mediated Islet Transplant Rejection . . . . . . . . . . . . . 28 Peter Ciechanowski

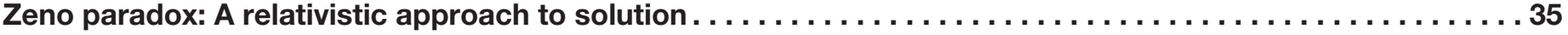
Matías A. O. Quiroga

\section{REVIEWS}

Organic Amnesia: A Diversity in Deficits

Christopher R. Madan

The Biggest Bugs: An investigation into the factors controlling the maximum size of insects 43 Delyle Polet 
My name is Cathy Danilec. Currently, I'm a University of Alberta Bachelor of Science graduate with a focus in biology and science psychology. In the future, I hope to obtain a Masters in Biomedical Communications - allowing me to illustrate textbooks, science journals, and create models or animations for classroom use.

For the front cover of this issue, the Eureka team was looking for an illustration unifying science and the world around us. Within this theme, we came up with the idea of a lab scene with focuses on different areas of science, specifically the biological sciences.

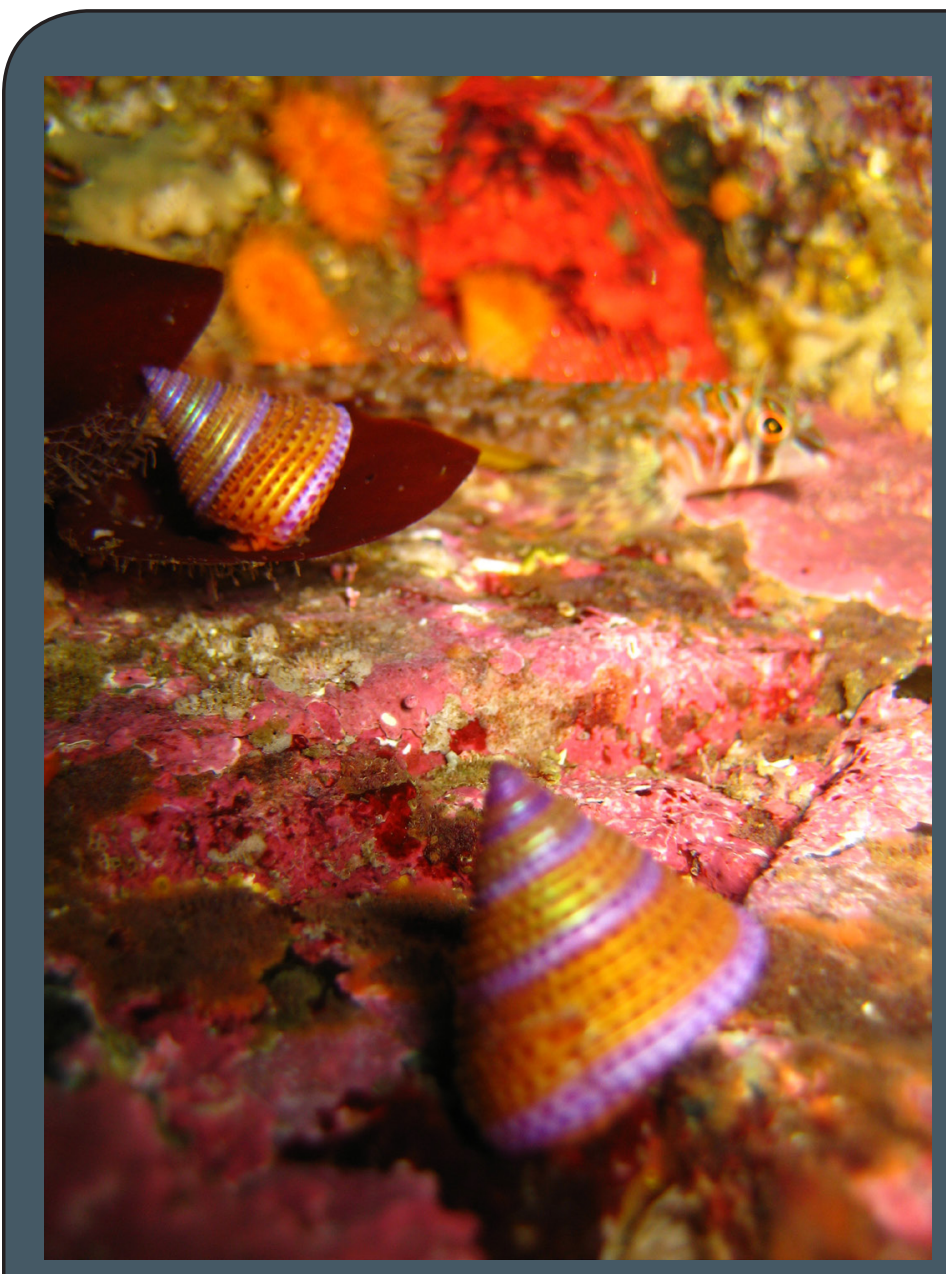

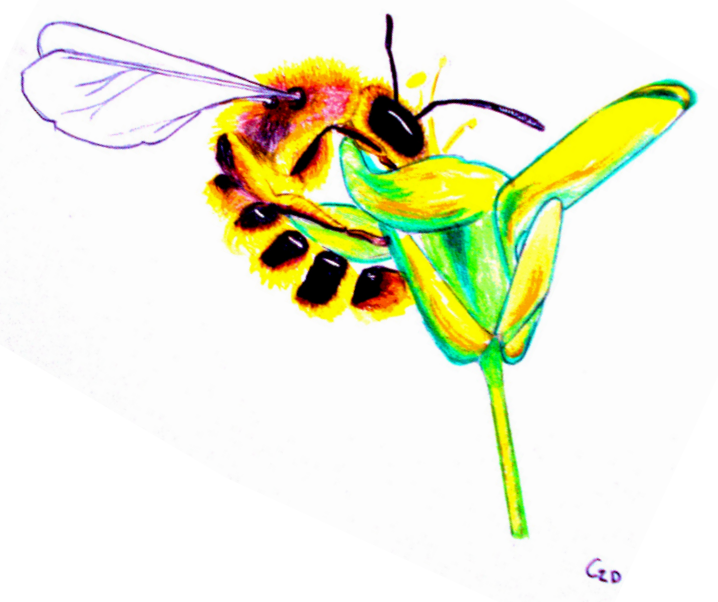

\section{ABOUT THE BACK COVER}

I grew up on an acreage outside of Lacombe, Alberta, with avid naturalists for parents. Our family vacations were most often into the backcountry of the Rockies, canoeing down some river, or off to explore the parks of Canada and the United States. As a result, at an early age I developed a fascination for the natural world and the sciences that let me learn how it worked.

Growing up on the prairies, there are not many tide pools, or even tides. I developed and interest in marine biology none-the-less when, in my grade 11 year, I was able to go on a school trip to the Bamfield Marine Sciences Centre (BMSC) that introduced me to marine biology. BMSC is a research station run by the five major universities in western Canada, including the University of Alberta. After that high school trip, I started applying to university programs that would get me back to the coast, and decided on the Animal Biology program at the $U$ of $A$.

In my fourth year, I was able to go back to Bamfield for the fall semester. Four months of immersive marine biology, with most classes spent out in the field doing everything from snorkeling with salmon during the fall run to dredging up deep water denizens to study, independently studying how octopus camouflage in the lab to hiking through the temperate rainforest to study organisms on remote beaches. After returning to Edmonton for the winter, I put off convocation and went back to Bamfield for one last course, scientific diving. Not a bad way to finish a degree!

The picture on the back cover of this journal was taken during one of my courses in Bamfield. I have no fancy equipment, just a cheap point and shoot digital camera, but even with that, I started developing an interest in photography, particularly underwater. I am hoping to pair my interest in marine biology with SCUBA diving, but where ever I go, my little camera will be close by because even in the most ordinary environment, there is amazing things going on in nature if you look through the right eyes. Or lens. 


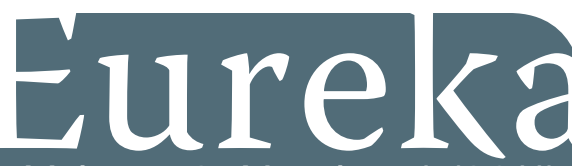

Volume 2, Number 1 (2011)

Eureka is a student-founded

and student-run initiative whose

mission is to promote the world

class undergraduate research

done in the faculty of science.

This journal offers undergraduate

scientists the unique opportunity

to share their discoveries with the

scientific world, while learning

the peer review process. Most

undergraduate students do not

get to experience the publication

process as part of their scientific

education. Eureka is an educational

institution, with a diverse team

of reviewers from many scientific

backgrounds. Through clear,

effective scientific writing students

will be able to work together with

faculty members to promote

University of Alberta science.

E-mail: eureka@ualberta.ca

Website: www.eurekajournal.com

Editor-in-Chief

Christopher Madan

Managing Editors

Yvonne Chen and Patrick Jones

Editorial Board

Yvonne Chen, Carmen Chu,

Ross Fitzsimmons,

Patrick Jones, Leiah Luoma,

Christopher Madan,

Davin Piercey, Helen Zakrzewski

Consulting Editor

Braden Teitge

Copy Editor

Dawn Wong

Layout Editor

Yvonne Chen

Founders

Braden Teitge

Christopher Madan

Patrick Jones

\section{EUREKA:}

A BEACON FOR UNDERGRADUATE RESEARCH

Many undergraduates consider research and graduate school as a possible next step after graduating with their BSc. However, many of these students have never tried research prior to embarking on this journey. Considering that getting a Master's degree is a major life decision, one should do their best to ensure that research is right for them.

Rather than postponing this journey until graduate school, 'inspired' undergraduates may want to begin early and try their hand at research while still an undergraduate. Most departments offer fourth year (usually '499') and even second year ('299') independent study courses, as well as Honour's degrees, where students can sample what research is like, rather than diving right into

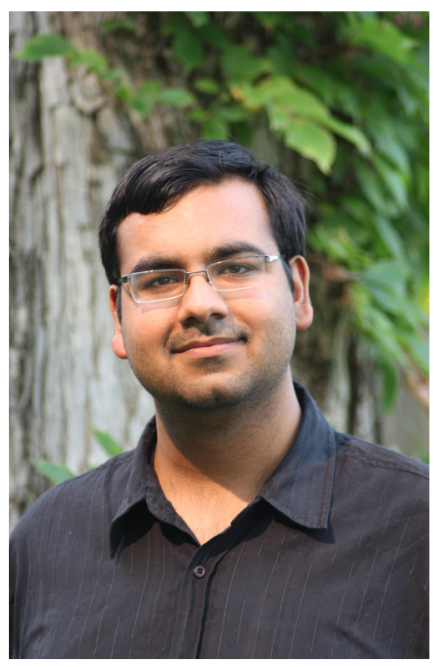
a Master's degree. Nonetheless, many students do not know what undergraduates can do within a research environment and that's where Eureka steps in.

Eureka's purpose is to showcase the research that undergraduate science students at the University of Alberta have done. First and foremost, I hope that Eureka serves to educate junior undergraduates of the possibilities available to them, by seeing what their slightly older peers have accomplished. As an added benefit, by publishing their work in a lightly peer-reviewed venue, senior undergraduates can experience the peer review process firsthand and include the publication on their CV, as well as in graduate school applications, potentially tipping the admissions committee in their favor.

Research done by undergraduates can range from such diverse topics as advances in islet transplantation (page 28), evaluating empathetic communication in Pharmacy students (page 10), and developing new technologies for interacting with computers (page 24). You can never start research too early, as demonstrated by one review paper by a Science 100 student (page 43). Additionally, Eureka also features several interview and editorial pieces done by our staff, such as on the role of the pharmacist in society (page 2) and on how to choose a graduate school (page 4). Eureka itself is also growing, as an article in our previous issue of Eureka sparked an undergraduate in Argentina to also submit his work to us (page 35).

If you are a senior undergraduate just finishing a research project, or are starting to work on one over the summer - talk to your supervisor about publishing your research work in Eureka.

Please visit http://www.eurekajournal.com for more details on publishing your research with Eureka.

Hopefully you feel inspired to do research after reading through this issue of Eureka - I know I sure am.

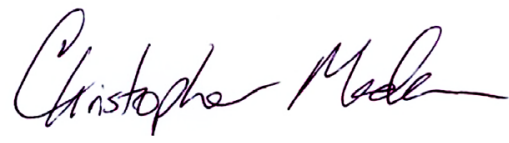

Christopher R Madan

Eureka Editor-in-Chief 


\section{THE EVOLVING ROLE OF THE PHARMACIST AND WHY YOU SHOULD BE ONE}

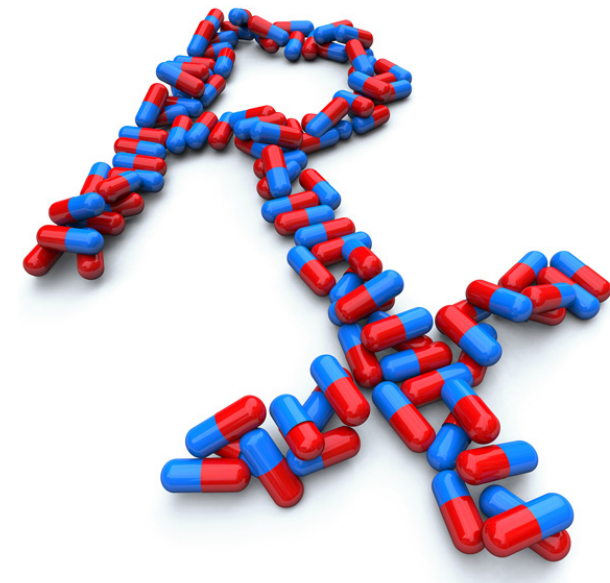

Traditionally, pharmacists have dispensed medications and filled/refilled doctor's prescriptions. However with advances in pharmaceuticals, pharmacists have become more involved with physician prescribing behaviours and practices today than ever before. Pharmacists now frequently modify doctors' prescriptions or even prescribe medications, review medications for safety and efficacy, educate the public on new medications on the market, and even investigate drug interactions and side effects. Advances in technology have allowed the pharmacist to perform laboratory tests, such as haemoglobin levels, and lipid profiles so they can give objective readings to patients to see how they are doing and suggest optimal drug therapy.

Specifically, Alberta pharmacists are now able to prescribe schedule 1 drugs, excluding benzodiazepines and narcotics, as well as administer some injections as per the 2006 Pharmacy and Drug Act. Because of Alberta's vast geographic area and rural nature, the pharmacist's increased role has allowed greater access of health services in remote areas where access to doctors is usually limited. Improved access can also be seen with chronic disease management in clinical or hospital settings. Although the Alberta pharmacist's focus is changing, the pharmacist remains an expert in the application and usage of pharmaceuticals.

\section{Why become a pharmacist?}

In my opinion, pharmacists work to live, meaning that their work gives them the financial assistance and the free time to carry out other life goals. They go into work and they come home not worrying about work. There is also the opportunity to own your own pharmacy and be your own boss. Also, most people who I have talked to do respect and think highly of their pharmacist. Being a pharmacist also allows you to work in any area that people live, so in a big urban centre to the smallest village. There is a need for pharmacists everywhere, so getting a job is eminent.

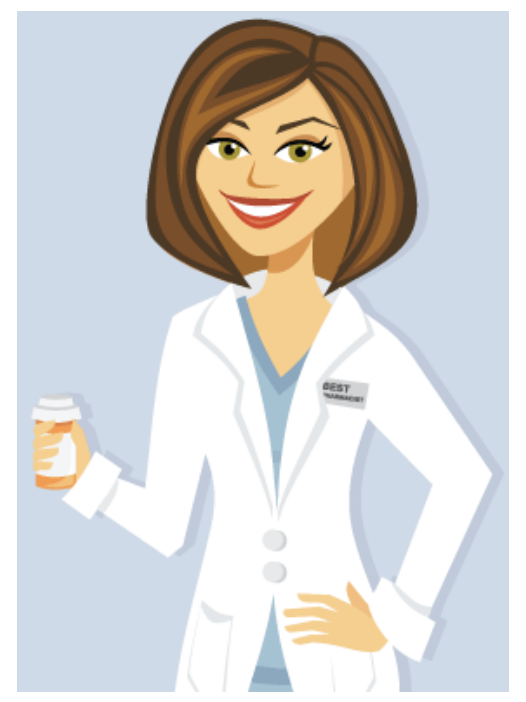




\section{Interested?}

There are a few pharmacy schools in Canada; the only pharmacy school in Alberta is at the University of Alberta. There are several courses which one must compete to become a pharmacist; all are listed on the pharmacy admissions website. Most students will usually complete the listed courses within the first two years of their undergraduate degree, but it is possible to complete the courses in only one year. And of course one must complete these courses with a high academic standing, above a 3.5 average GPA is considered to be a competitive average. However, if you happen to party too much in first and second year and your GPA is not above 3.5, do not worry. If you complete more than two years of university the admissions committee will weight your performance during your last two years of study. The only other documentation that you need to submit is the letter of intent, which outlines your understanding of the profession, your volunteer and work experience, and usually an ethical question. The letter should be very detailed description yourself and should demonstrate that you are a rounded personable individual who excels in activities outside of school. There is no interview and no admission test to write, which makes the $U$ of $A$ application process that much less stressful. Check out the Faculty of Pharmacy and Pharmaceutical sciences website site @ http://www.pharm.ualberta.ca, and see where it may take you!

Patrick Jones

BSc (Specialization) Ecology and Pharmacy first-year student

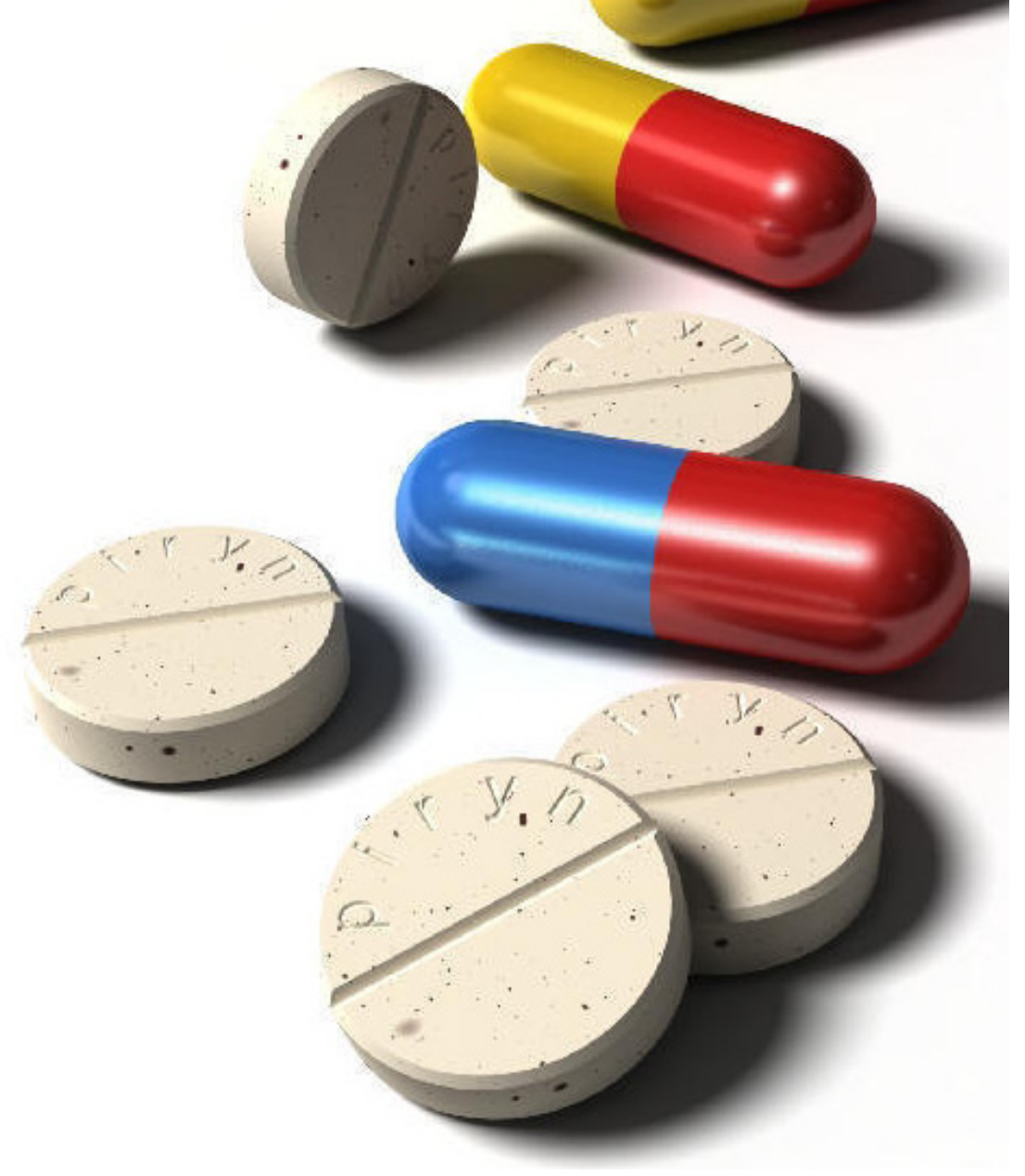




\section{CHOOSING GRADUATE STUDIES}

Braden Teitge

First of all, what do you want to do! What really interests you? What subject in your undergraduate courses made you sit on the edge of your seat? That is the easy part; the more difficult part is choosing the right graduate school and the right professor.

Now define your area. If you want to stay in Canada, you have many choices of institutions that are doing some really groundbreaking work. Keep in mind though, that you are in a great place already, as both of the Universities of Alberta and Calgary boast tremendous graduate programs. If you have your heart set on going overseas for graduate studies, start looking early, as there are fewer studentship's available for overseas studies. These studentships are underutilized though, and many of the tri-council agencies (NSERC, CIHR and SSHRC) encourage these applications. Keep in mind that it may be easier to perform research abroad later in your career, after attending scientific meetings and speaking with potential supervisors in a more cost-effective manner. If you are thinking that you want to stay in Canada, you can slim your search down to the respective fields of study quite easily. Every major university has a list of faculty members and their departments, and it is relatively simple to navigate the websites to see biographies on the faculty members, their publications, and contact information.

But before you rush into anything take a step back and analyze your potential supervisor for a number of criteria that lead to a successful graduate program. First of all, does he or she have funding? Do they have grants right now that will let you perform the experiments you want to perform? The major granting agencies usually have published lists of the grant money they gave out. Also, most professors will tell you what funding they have - just ask. Now that you have finances sorted out, think about the lab environment that you want to be in. Many of the most successful, high-profile researchers have done amazing things in their careers, but in a sea of 25 other trainees and students, would you have the chance to learn from this person? Finally, what about the graduate program you would enter into. Many faculty members are part of a single department or research group, so take a close look at the program, the required courses, and the level of funding that it promises its students.

Now that you have your list of prospective supervisors down to a handful, it is now time to contact them. A wise professor once told me that when students contact him, the first thing he looks for is have they done their homework. Many students "broadcast their curriculum vitae (CV)" to a list of 50 supervisors with a generic message such as "I am interested in being your graduate student. I have amazing grades. Take me". It doesn't work quite like that. What many professors look for, is 'does he or she know what my lab currently studies' and 'has this student read my publications'. This should be a high priority for you, and relatively easy since you're looking at labs doing research of interest to you.

If you really cannot decide on a research path, one of the most useful things you can do is ask a professor of yours. Professors know who does good research, and they know who is good at training students. Often, they might be the critical link that you need to get into his or her lab, and hey, it never hurts to ask. Even if you give them a broad area, such as "[Dr. Prof], I am interested in [broad research category], and would like to pursue graduate studies on this topic in the fall. Could you recommend somebody that does good research on the subject?" 
When you finally get to the big moment of asking a prospective professor if they would meet with you to discuss graduate school, let them know that you are qualified! Many professors get dozens of requests a month, so it is important to let them know that you are an excellent student in your first email. Here is an example of what this email might look like:

Dear Dr. Supervisor, I am [your name]. I will be graduating in May with a BSc in [your subject] from the University of Alberta and I am looking for graduate studies in the fall. I have taken numerous courses in [relevant area], which explains my interest in your work on [their research]. I have read your publications on [their research] and [description of it]. I would very much like to meet with you to discuss your current research interests and how I can be part of it. I have a great deal of pertinent experience, as I have worked in [previous researcher's] lab. I feel I would be competitive for several scholarships such as Alberta Innovates and NSERC, so there is a good chance I would come to your lab with my own funding. I have attached my CV so you can evaluate my qualifications in more detail. Dr. [previous supervisor] has also agreed to serve as a reference, please feel free to call him/her.

\section{I look forward to your reply.}

When interviewing, come prepared to show that you know what you are talking about. Make sure you have done your homework on their research, and do not be afraid to ask questions. Graduate studies is about asking the right questions, so you might as well start now. If you are undecided about a particular lab, or trying to decide between more than one, the best way to get to know the place is to talk to the current graduate students. Straightforward questions such as 'do you get along with the boss', and 'what is the working environment like', are often answered honestly by the current graduate students. Ask for their email to contact them later if you have more pressing questions.

Other criteria that you may want to consider would be the prestige of the graduate program, their education standards, access to building facilities, and cost of the program (don't forget what the stipend is set at and what housing is available). When deciding on when to enter graduate studies, it is often the graduate coordinators that know all of the information. Keep track of your application deadlines, and equally important, the scholarship deadlines that are dreadfully early! Some of these scholarships allow you to apply with more than one supervisor in mind, and some even allow you to transfer between labs if you change your mind. It is very important to apply for these scholarships, as they will greatly increase your chances of getting more scholarships later in your research career. 


\section{HOW DONATED BLOOD IS TESTED: AN INTERVIEW WITH CANADIAN BLOOD SERVICES}

\section{Eureka Team}

Sherwin Dziwenka, Regional Manager of Western Donor Testing, outlines the testing protocol undertaken by Canadian Blood Services when screening the various blood products collected.

\section{i) Who is responsible for testing of blood products collected by Canadian Blood Services?}

Western Consolidated Donor Testing Laboratory tests donor samples for all mandatory and supplemental tests from British Columbia, Alberta, Saskatchewan, Manitoba and the Yukon Territories.

\section{ii) For which diseases are the collected blood products tested?}

The collected blood is tested for the following infectious diseases: Syphilis, Human Immunodeficiency Virus 1 and 2, Hepatitis B and C, Human T-Cell Lymphotropic Virus I and II, and West Nile Virus. These tests are mandatory and must be performed on every CBS donor sample.

There are 2 additional tests that may or may not be performed, which are dependant on:

- specific recipient requirements, which may result in the collected blood being additionally tested for Cytomegalovirus;

- and donor history, which may result in the collected blood being additionally tested for Chagas.

\section{iii) How is testing on collected blood products} undertaken?

Testing for the purpose of blood donor screening must meet regulatory requirements and quality assurance expectations. Testing is required for each donation and must use a concurrent drawn sample.

Serological tests are initially performed singly; nonreactive results are interpreted as negative, allowing release of the component for transfusion. Reactive results are repeated in duplicate: if both repeat results are nonreactive, then the result is interpreted as negative. If one or both repeat results are reactive, then the test result is considered "repeat reactive" and the associated blood donation cannot be used for transfusion. Supplemental tests are performed on repeat-reactive samples primarily for donor notification and counseling purposes.

NAT testing begins with a number of blood samples being "pooled" together in the laboratory. If the result of a pool of samples is negative, all samples in the pool are negative. If the pool is positive, then the samples are tested in smaller pools and then individually until the actual positive sample is identified.

Questions written by the Eureka Editorial Team with answers provided by Sherwin Dziwenka, Regional Manager of Western Donor Testing for Canadian Blood Services.

\section{Eureka}

Volume 2, Number 1 (2011)

With each unit of blood, platelets or plasma collected there are 5 samples (4 plasma samples and 1 serum sample) collected and sent to laboratory for testing.

First plasma sample is sent to the Blood Group Serology Laboratory to perform:

- $\mathrm{ABO}$ Grouping

- Rh Type

- RBC Antibody Screen

- Syphilis test

- Cytomegalovirus test, when applicable

Second plasma sample goes to the Nucleic Acid Testing Laboratory to perform:

- West Nile Virus RNA test

Third plasma sample goes again to the Nucleic Acid Testing Laboratory to perform:

- Hepatitis C Virus RNA test

- Human Immunodeficiency Virus -1 RNA test

Fourth plasma sample is called "Retention Sample" and is used for any confirmatory or Chagas testing if required. Chagas testing is only performed on samples for a donor that answered yes to one or more of the 3 Chagas risk questions during donor registration.

The one serum sample goes to Transmissible Disease Laboratory to perform:

- Antibody to Human Immunodeficiency Virus Type 1 and 2 test

- Hepatitis B Surface Antigen test

- Antibody to Hepatitis C Virus test

- Antibody to Human T-Cell Lymphotropic Virus Type I/II test

- Antibody to Hepatitis B Virus Core Antigen test

\section{iv) Please explain in detail one particular test.}

ABO grouping

The determination of an $\mathrm{ABO}$ blood group is done by demonstrating the presence or absence of antigens $A$ and/or $B$ on the surface of human red blood cells and by detecting the presence or absence of anti-A and/or anti-B antibodies in the plasma. If an individual has an antigen on the surface of his/her red blood cell then they don't have the corresponding antibody in the plasma. It is therefore appropriate to identify the erythrocyte antigens using known anti-A and anti-B, then to confirm the results by verifying the presence of the corresponding antibodies in the plasma from the test blood using known red blood cells $A 1$ and $B$, also known as reverse grouping. There is also testing of red blood cells with Anti-A,B. It is an additional check for the presence of $A$ and $B$ antigen as well as $a$ 
facilitator in recognition of certain weak subgroups. The test is based on the principle of agglutination and pattern recognition. When red blood cells have the antigen on their surface, agglutination will occur with the reagent containing the corresponding antibody. Agglutination with an antibody indicates the presence of the specific antigen. If there is no agglutination it means that the red blood cells lack the antigen. This is how you determine if somebody is blood group: $A$ ( $A$ antigen is present), $B$ ( $B$ antigen is present), $A B$ (both $A$ and $B$ antigens are present) or $O$ (neither $A$ nor $B$ antigen is present).

\section{v) How long, on average, does the entire testing process} take?

The entire testing process takes approximately 12 hours from the time sample arrives to the Sample Management Lab till the time all the results are ready.

vi) If blood products collected from an individual test positive on a particular test, what is done with these blood products?

If this positive result is for a transmissible disease (eg HIV), the blood products are destroyed by incineration.

\section{vii) What does a "false positive" result mean? What is done with blood products that test positive falsely?}

The tests performed by Canadian Blood Services are extremely sensitive and they sometimes produce "false positive "results. This occurs in a small number of donors when a substance or substances in their healthy blood trigger a reaction. To determine if reactive results are truly positive, all units that produce repeat reactive results with screening tests are subjected to additional confirmatory testing. If confirmatory testing produces a negative result, the unit is considered a "false positive". A negative result in confirmatory testing is reassuring news for the healthy donor.

Nevertheless, no unit that produces a repeat reactive result with a screening test is ever transfused. Canadian Blood Services takes extra precautionary measures to ensure safety. It destroys any blood unit that tests "false positive" and maintains records so that these individuals are not accepted for blood donations in the future.

viii) Often the term window period is referred to during testing of blood products. What does this term mean?

Most traditional screening tests require the presence of antibody to trigger a positive test reaction. The period of time between initial infection and detection of antibodies is called the "window period".

\section{ix) What steps are undertaken to maximize safety for} recipients, despite the existence of a 'window period'?

Selection of eligible blood donors is a critically important step to maximize the safety and integrity of the blood supply. Selection of healthy donors by interviews and limited physical examination represents the first protective layer against infectious disease transmission.

Deferral of donors with certain past or current medical conditions, donors who engage in activities known to increase risk to the blood supply, immigration background, travel histories or specific physical findings minimizes the risk of collecting blood, plasma or platelets contaminated with transmissible agent.

In addition to screening out individuals whose health or high risk activities may put recipients at risk, stringent laboratory tests for infectious diseases are conducted on each unit of blood, plasma or platelets.

x) What is nucleic acid testing (NAT)? Why is it being increasingly used in Canadian Blood Services' testing procedures? What are its benefits versus traditional antibody/antigen testing?

Nucleic Acid Testing (NAT) detects the presence of viral genetic material in donor samples. Detection of viral RNA or DNA involves three steps: extraction, amplification and detection. Extraction occurs first and is followed by capture of viral RNA or DNA onto a capture molecule that is immobilized on a solid phase or onto magnetic particles in a solution. Once immobilized, the impurities are removed by a series of wash steps. Prior to amplification, viral RNA must be converted to DNA; this is accomplished by the enzyme, reverse transcriptase. Amplification of the DNA then can occur through multiple intermediates. Detection of the amplified product occurs by capture of the amplified DNA on nitrocellulose or by enzyme immunoassay or chemiluminescence.

NAT works by detecting the low levels of viral genetic material present when an infection occurs but before the body begins producing antibodies in response to a virus.

NAT significantly reduces the "window period". It allows for earlier detection of the infection and thus further decreases the possibility of transmission via transfusion. That is why it is increasingly used by CBS.

NAT is used in addition to antibody tests. Some individuals infected with Hepatitis $\mathrm{C}$ or HIV may be NAT - negative if the amount of virus present has fallen below detectable levels. In such cases antibodies to the virus would still be detected by current more traditional tests for Hepatitis C or HIV.

\section{xi) Whose blood is tested? Are blood products from} individuals who have previously donated also tested?

No matter how many previous donations, each unit of blood, plasma or platelets collected is tested as outlined above. Every donation has to have all the mandatory tests completed before any products made from that donation can be released for use. If any of the mandatory tests can't be completed on a given donation all the products from that donation are destroyed.

xii) How important are these testing procedures to the safety of Canada's blood supply?

It is possible for infectious agents to circulate in the bloodstream for weeks, months, or even years without causing any symptoms. Infected persons may unknowingly present as blood donors, and their donations may be accepted. In these cases, the next barrier to maintain the safety of the blood supply is effective blood donor 
screening tests. Fortunately, sensitive tests for selection of donors are available and their use is mandated whenever blood is collected for the purpose of transfusion.

xiii) Is there any piece of information you believe our reader's should know about the testing of blood products at Canadian Blood Services?

Safety is the paramount concern of Canadian Blood Services. Because blood is a biopharmaceutical product, it must be manufactured within very strict regulatory standards using Good Manufacturing Practices. As a result of Canadian Blood Services commitment to meeting national and international safety standards, Canada's blood supply is recognized as one of the safest in the world. Donor Testing has a very important role within the organization to ensure the safety of blood supply in Canada. We use the most advanced "state of the art" methods of testing blood for the presence of infectious diseases and blood groups.

There is a constant need for blood. To book your appointment please call 18882 DONATE.

For more information on blood awareness, contact the University of Alberta Blood Awareness and Donation Students Association (BADSA) at

http://www.ualberta.ca/ badsa/

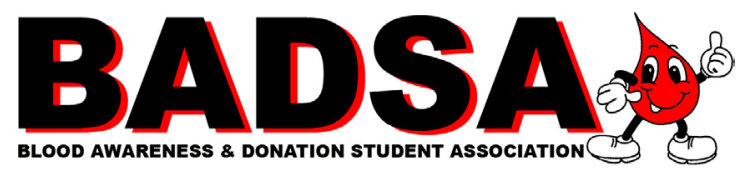

\section{Discover Undergraduate Research}

\section{Do you want to:}

- make your undergraduate degree

stand out?

- develop connections with

faculty?

- investigate topics you're

passionate about?

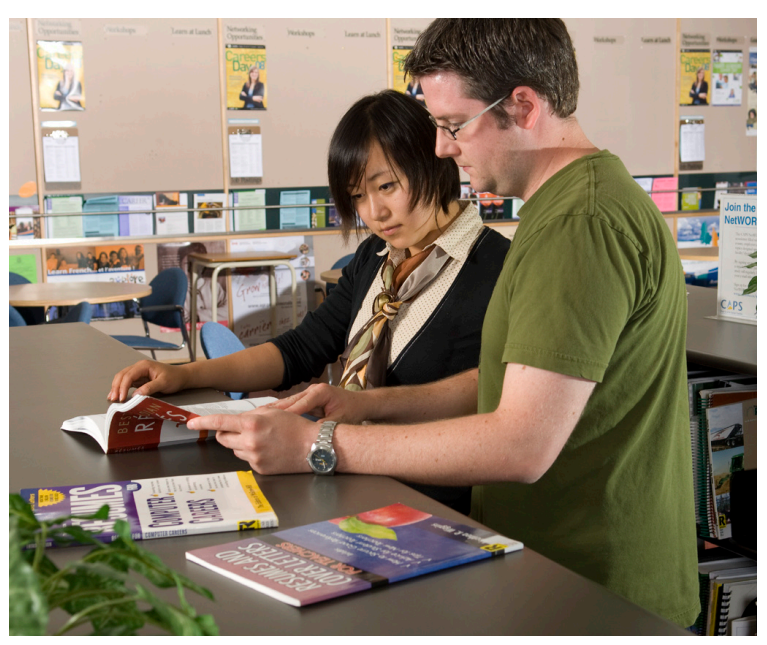

Getting involved in undergraduate research is a great way to do these things and more. Visit CAPS and get ideas about how to get involved. 


\section{BIOFEEDBACK AS A PHYSIOLOGICAL TREATMENT FOR TRAUMATIC PSYCHOLOGICAL INJURIES}

Eureka

Volume 2, Number 1 (2011)

Lauren Alston

Department of Psychology, University of Alberta

For over a century it has been argued that the psychological effects of trauma are often expressed as physiological changes in the biological stress response. Research supports that biofeedback can be used as a tool that increases an individual's awareness of their physiological response to overwhelming sensations and teaches them how they can take control of it. Literature on trauma suggests the need for physiological-awareness therapy, meanwhile literature on biofeedback indicates that it is a technique that focuses specifically on physiological awareness. Biofeedback is a therapeutic tool that uses sensors to record physiological changes in the stress response and provides an individual with feedback on their current bodily state. However, little research has linked the potential benefits of using biofeedback, in conjunction with other treatments, to help traumatized individuals recover. In 2006, van der Kolk pointed out that little is known about how people can learn to regulate their physiological arousal. He argued that a lack of arousal modulation is a dominant issue in traumatized individuals. In line with this, Hopper et al. (2007) posited that humans have a greater self-regulatory potential than other mammals but that the efficiency of regulation can be impaired in individuals with Post-Traumatic Stress Disorder (PTSD). van der Kolk (2006) also indicated that a traumatizing experience involves an individual's inability to engage in an action pattern that results in avoidance of the trauma. When a traumatic experience becomes unavoidable, a person can become overwhelmed at the futility of their situation and lose their capacity to use their emotions as guidelines for successful action. van der Kolk further suggested that therapeutic techniques need to be explored in the realm of reprogramming the automatic physiological responses evoked by emotions. Findings show that biofeedback targets an individual's awareness of their internal physical sensations, which have a reciprocal relationship with a person's emotional experience. The need for an individual to cope with trauma at a physiological level enhances the value of biofeedback as a necessary tool in this process. Since traumatized individuals have deficits in understanding their own physiology, biofeedback can give them the insight they require to regain their sense of self-control (Ogden, Minton, \& Pain, 2006). Therefore the implications are that biofeedback can be used in conjunction with other trauma-related therapies because it addresses the underlying physiological issues of trauma.

\footnotetext{
References

Hopper, J., Frewen, P., van der Kolk, B., \& Lanius, R. (2007) Neural correlates of reexperiencing, avoidance and dissociation in PTSD: Symptom dimensions and emotion dysregulation in responses to script-driven trauma imagery. Journal of Traumatic Stress 20(5): 713-725.

Ogden, P., Minton, K., \& Pain, C. (2006). Trauma and the Body: A sensorimotor approach to psychotherapy. New York: W.W. Norton \& Company. van der Kolk, B. (2006). Clinical Implications of Neuroscience Research in PTSD (Vol. 6). New York: New York Academy of Sciences.
} 


\section{SELF-ASSESSMENT AND OBSERVER RATING OF EMPATHETIC COMMUNICATION IN PHARMACY STUDENTS}

Eureka

Volume 2, Number 1 (2011)

Lisa M. Guirguis \& Enoch Ng

Faculty of Pharmacy and Pharmaceutical Science, University of Alberta

\section{Introduction}

Little research has been conducted on empathy assessment in pharmacy education. The study objectives are to 1) compare pharmacy students' self-assessments and observer ratings of empathetic communication in simulations with standardized patients, 2) compare pharmacy students self-reported empathy with self-assessment and observer ratings, and 3) examine gender in empathetic communication.

\section{Methods}

First year pharmacy students enrolled in a communications course completed the empathic concern and perspective taking scales from the Interpersonal Reactivity Index (IRI) and participated in a video recorded patient interaction focusing on initial medication use. Students used the Empathic Communication Coding System (ECCS) to rate the first empathic opportunity in their video recording. Two researchers separately rated the video recordings using the ECCS for the empathic opportunity identified by the students and discrepancies were discussed until consensus was reached. Description statistics, correlations, and T-tests were used as appropriate to analyse the data.

\section{Results}

Analyses were performed on 63 students (class of 131) who consented and had complete data. Students rated themselves higher on the ECCS (3.6, SD=1.4) when compared with observers $(2.6, S D=1.1)(T$-test, $p<0.05)$. There were no statistically significant correlations between the IRI self-report and either student or observer ECCS ratings. Male students rated themselves lower on the ECCS $(3.2, S D=0.3)$ than females $(3.8, S D=0.8)$ while there were no gender differences in observer ECCS scores (male: 2.7, $\mathrm{SD}=1.5$; female: $2.6, \mathrm{SD}=1.3$ ).

\section{Discussion}

Pharmacy students were not able to accurately assess their own empathic communication. Our results indicate that students' self-report empathy was not correlated with behaviour. Observers found no difference between male and female empathetic communication; although males has lower self-assessment than their female colleagues. Further work is required to find appropriate methods for evaluating student empathy and developing accurate self-assessment of empathic communication.

Note: This abstract was presented at the European Association on Communication in Health Care in Verona, Italy from September 5-8, 2010. 


\title{
STEROL CHEMICAL STRUCTURE AND CONFORMATION INFLUENCE THE THERMOTROPIC PHASE BEHAVIOUR OF DIPALMITOYLPHOSPHATIDYLCHOLINE BILAYERS
}

\author{
Matthew G. K. Benesch, David A. Mannock, \& Ronald N. McElhaney \\ Department of Biochemistry, School of Molecular and Systems Medicine, Faculty of Medicine and Dentistry, University of Alberta
}

\section{Abstract}

Studying the nature of interactions between the sterol ring system and neigbouring phospholipid molecules is important for our understanding of the properties of sterols in biological molecules and the role of such interactions in many disease processes. In this project, the thermotropic phase behaviour of binary dipalmitoylphosphatidylcholine (DPPC)/sterol mixtures with different sterol ring configurations ( $\mathrm{C} 5,6$ double bond, $5 \alpha-\mathrm{H}$ and $5 \beta-\mathrm{H}$ orientation and either $3 \alpha-\mathrm{OH}, 3 \beta-\mathrm{OH}$, 3-ketone functional groups) was investigated using differential scanning calorimetry (DSC) and was compared to earlier studies of cholesterol/ and epicholesterol/DPPC mixtures. Given the differences in the thermodynamic parameters obtained from these mixtures and their associated changes in bilayer stability and miscibility, it is clear that changing the sterol chemical configuration has a significant effect on bilayer properties. Any sterol molecule whose ring structure deviates from that of cholesterol is unlikely to be fully miscible in mammalian membranes.

\section{Introduction}

The lipid bilayers of biological membranes contain a wide range of phospho- and glycolipids, as well as other amphiphilic molecules differing in their chemical structure. Of those, sterols such as cholesterol (Chol) are among the most commonly identified membrane constituents, modulating the physical properties and lateral organization of the plasma membrane lipid bilayer. Chol has an equatorially orientated $\mathrm{C} 3-\mathrm{OH}$ group in ring $\mathrm{A}$ of the steroid nucleus (Fig 1), which has been identified to be planar and rigid, a double bond at $\mathrm{C} 5,6$ in ring $\mathrm{B}$, and an alkyl side-chain at $\mathrm{C} 17$ of the cyclopentane ring. The small polar headgroup and large hydrophobic ring and side-chain anchor Chol in the phospholipid bilayer with the long axis of the sterol molecule lies parallel to the lipid acyl chains and the polar headgroup sitting in the bilayer interface. Biological membranes are fluid at physiological temperatures but are thought to be regionally heterogeneous, containing lipid microdomains or rafts, which are enriched in tightly-packed, structurally
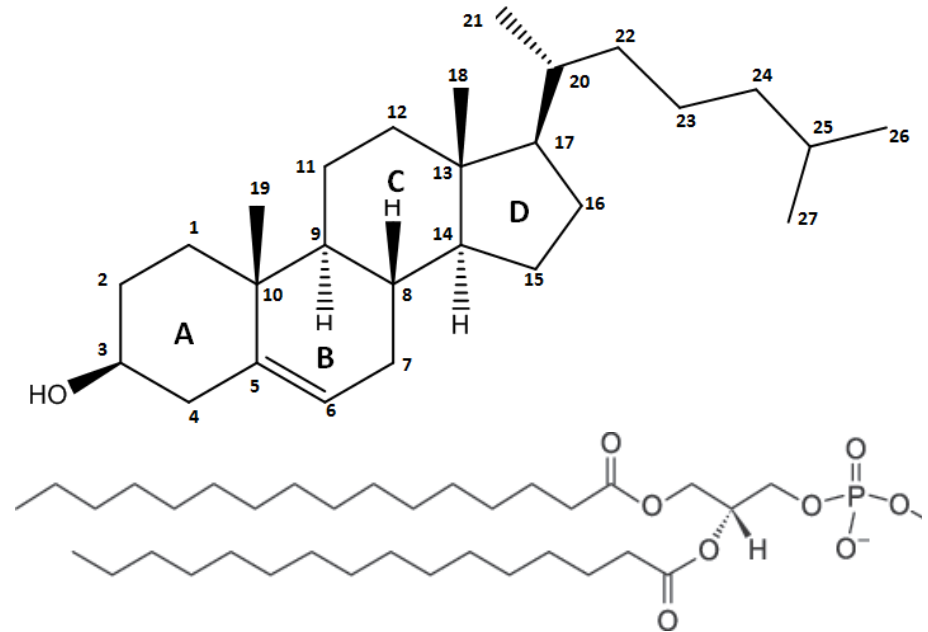

Figure 1. The chemical structure of a typical sterol (top), cholesterol, with both carbon atom numbering and ring identification. This study investigates the effect of changes in the chemical structure of the steroid ring system on ring conformation and electronics. Changing the stereochemistry at C-3 and removing the carbon 5-6 double bond introduces a new stereocentre at C-5. The chemical structure of 1,2-dipalmitoyl-3-sn-phosphatidylcholine (bottom), the phospholipid matrix used in this study. important lipids, such as sphingomyelin (SpM) and Chol. These ordered microdomains are surrounded by regions of more disordered lipids, enriched with phosphatidylcholines (PCs) and other phosphoglycerolipids. Lipid rafts from diverse natural sources contain sterols with different chemical configurations, whose structure is supposedly optimized to maintain the permeability, fluidity, elasticity, and curvature of the host membrane. However, relatively little is understood about how systematic changes in sterol structure affect those physical parameters through their interactions with surrounding lipid molecules. Nevertheless, the various sterol structures differ in their effects on the chain-melting phase transition of the host membrane lipids and in their condensing and ordering of the liquiddisordered bilayer to create liquid-ordered domains, which differ in their bulk physical properties relative to a sterolfree bilayer composition.

Several important human and mammalian diseases have been associated with the membrane sterol content and with lipid rafts in particular. These include neurodegenerative diseases such as Parkinson's and Alzheimer's (1-3), viral- (4-6) and prion-associated diseases $(7,8)$, bacterial infections (9), as well as diabetes (10) and some cancers $(11,12)$, all of which have a common site of action on the cell membrane: the lipid rafts (13). In these diseases, metabolic dysfunctions or the binding of infectious agents disrupt the cell biochemistry by means of associated physical mechanisms (11-14), eventually leading to changes in cell metabolism and, through changes in lipid signaling pathways, producing either cell growth or death and disease manifestation (15). Recent studies indicate that once the above processes begin, lipid raft reorganization occurs because of the separation of the lipid constituents and their lateral redistribution $(16,17)$, together with changes in the localized concentration of important proteins and cell surface antigens on the membrane surface $(17,18)$. However, how changes in lipid and sterol structure influence these demixing processes are not completely understood.

Our understanding of the nature of interactions between sterol and lipid molecules in biological membranes is also 
incomplete. While there have been many investigations into the effects of Chol on the gel/liquid-crystalline $(\mathrm{Pa}, /$ $L \alpha)$ phase transition of model phospholipids with a range of headgroup structures and with either saturated or unsaturated acyl chains (19-26), there have been relatively few studies in which the sterol tetracyclic ring and isooctyl side-chain have been systematically varied. This situation arises because of the limited number of natural sterols that are commercially available at reasonable cost and the absence of a substantial pool of sterol structural motifs among those products. Thus, it is difficult to understand the roles played by variations in the number of functional groups, their position, and orientation in the tetracyclic ring system and the alkyl side-chain and, consequently, the changes in the conformational and electronic contributions arising from each sub-structure. All of these contributions affect the sterol molecular conformation and modify the bilayer physical properties.

Previous work in our laboratory used differential scanning calorimetry (DSC) to study the thermotropic phase behavior of binary Chol and epicholesterol (EChol; axial C3-OH group)/dipalmitoylphosphatidylcholine (DPPC) mixtures (24). Summarized briefly, the Chol/EChol study indicated that the incorporation of EChol is more effective than Chol in reducing the enthalpy of the pretransition of DPPC and is initially more effective than $\mathrm{Chol}$ in reducing the enthalpies of both the sharp and broad components of the main phase transition of DPPC, which arising from the sterol-poor and sterol-rich regions, respectively. However, at 30-50 mol\% EChol sterol concentrations, EChol becomes less effective than Chol in reducing the enthalpy and cooperativity of the main phase transition, such that although at sterol concentrations of $50 \mathrm{~mol} \%$, EChol, unlike Chol, does not completely abolish the cooperative hydrocarbon chainmelting phase transition of DPPC, it does not appear to form a calorimetrically detectable micro-crystalline phase at higher sterol concentrations. This illustrates the importance of Chol in biological membranes. Spectroscopic and other results indicate that monomeric EChol is less miscible in DPPC bilayers than is Chol at higher sterol concentrations, but perturbs their organization to a greater extent at lower concentrations, probably due to the lower position of EChol in the bilayer interface.

This evolving molecular perspective from previous work on binary sterol/DPPC mixtures is extended here to include the cholesterol ketone (5-cholesten-3-one, CholK), and four sterols and two steroids with no double bond in ring $B$ with the $5 \alpha-\mathrm{H}$ and $5 \alpha-\mathrm{H}$ conformation at the junction of rings $\mathrm{A}$ and $\mathrm{B}$ and either a $3 \mathrm{a}-\mathrm{OH}, 3 \alpha-\mathrm{OH}$, or 3-ketone functional group. Two- and three-dimensional structures of these sterols and steroids are shown in Figs 2-4. The $5 \alpha-\mathrm{H}$ and $5 \alpha-\mathrm{H}$ sterols (corprostanol and epicoprostanol, respectively) are found in sewage and marine sediments and soil, and are thought to be Chol reduction products produced by gut and soil bacteria (27). The steroids are oxidation products of the corresponding alcohols and are early intermediates in the biosynthesis of bile acids and hormones (28).
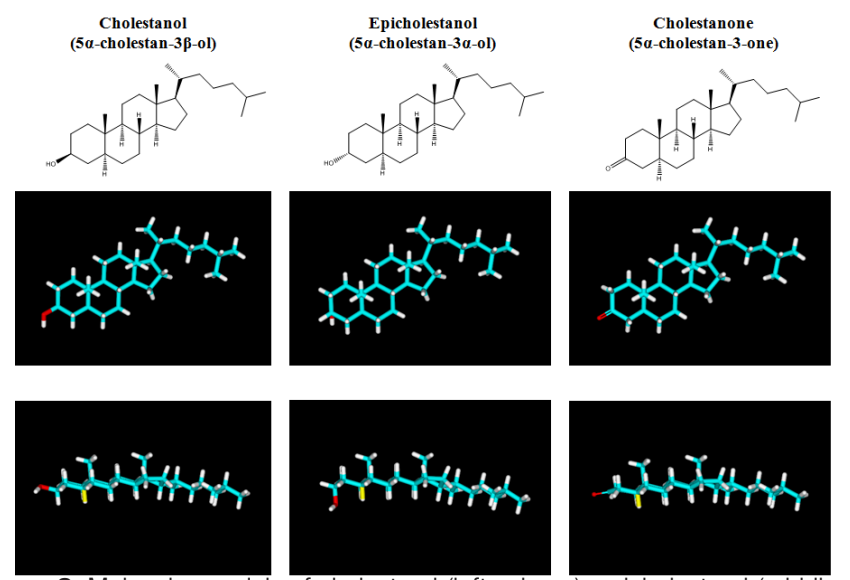

Figure 2. Molecular models of cholesterol (left column), epicholesterol (middle column), and keto-cholesterol (right column). The top row panels show the 2D structure of these sterols, the middle row panels show views normal to the plane of the sterol ring and the bottom row panels show views parallel to the plane of the sterol ring. The C-3 hydroxyl and ketone groups are colored in red. The molecules were minimized using DSviewer 5.0 (Accelrys Software Inc., San Diego, CA).

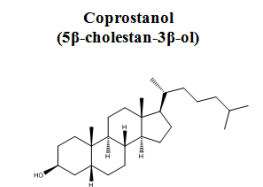

$$
\underset{(5 \beta-\text { cholestan-3a-0l) }}{\text { Epicoprostanol }}
$$

$\underset{\text { (5p-cholestan-3-one) }}{\text { Coprostane }}$
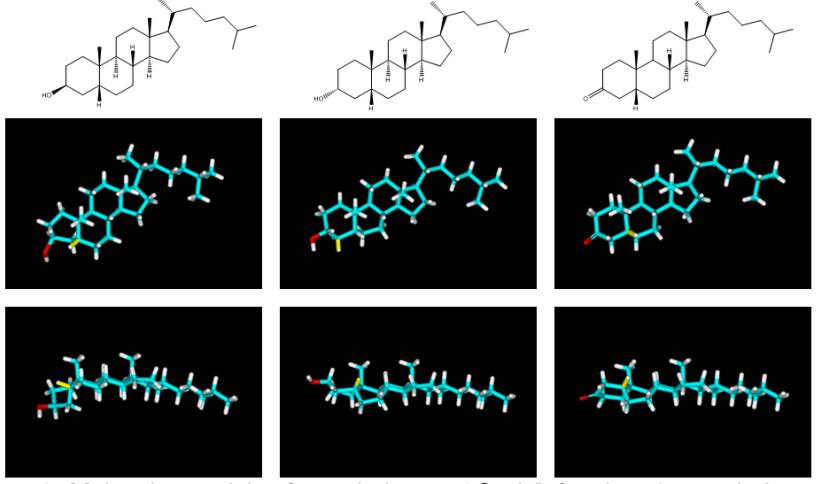

Figure 3. Molecular models of $5 \alpha$-cholestan-3 $\beta$-ol (left column), $5 \alpha$-cholestan$3 \alpha-0 l$ (middle column), and 5a-cholestan-3-one (right column). The top row panels show the 2D structure of these sterols, the middle row panels show views normal to the plane of the sterol ring and the bottom row panels show views parallel to the plane of the sterol ring. The C-3 hydroxyl and ketone group are colored in red and the $5 \alpha$ proton is highlighted in yellow. The molecules were minimized using DSviewer 5.0 (Accelrys Software Inc., San Diego, CA).
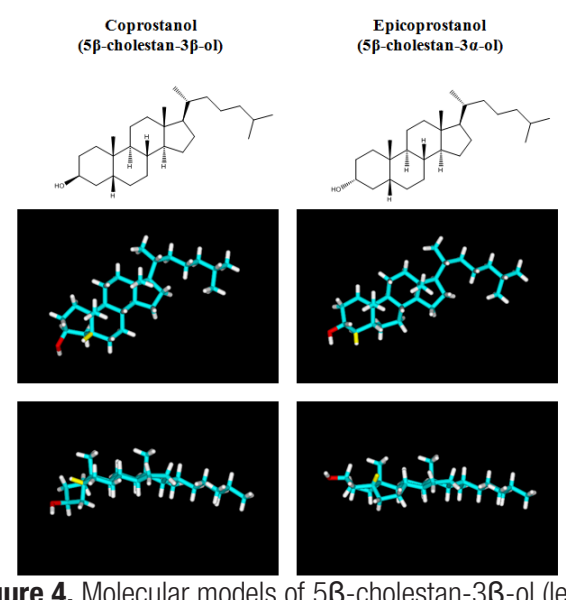

$$
\underset{(5 \beta-c h o l e s t a n-3-o n e)}{\text { Coprostanone }}
$$
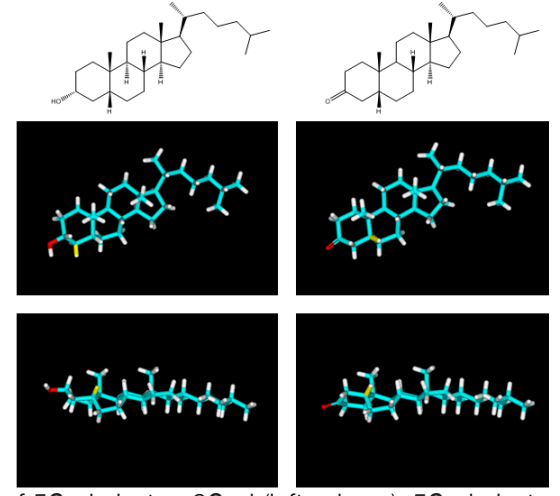

Figure 4. Molecular models of $5 \beta$-cholestan-3 3 -ol (left column), $5 \beta$-cholestan$3 \alpha-0 l$ (middle column), and 5 $\beta$-cholestan-3-one (right column). The top row panels show the 2D structure of these sterols, the middle row panels show views normal to the plane of the sterol ring and the bottom row panels show views parallel to the plane of the sterol ring. The C-3 hydroxyl and ketone group are colored in red and the $5 \alpha$ proton is highlighted in yellow. The molecules were minimized using DSviewer 5.0 (Accelrys Software Inc., San Diego, CA). 


\section{Experimental Procedures}

The DPPC and Chol (both 99\% pure) were both obtained from Avanti Polar Lipids (Alabaster, AL), whereas the remaining sterols and steroids (all 98\% pure by TLC) were supplied by Steraloids Inc. (Newport, RI). All organic solvents were analytical grade and were redistilled before use. Hydrated samples for DSC were prepared exactly as described previously (23). The dry DPPC:sterol films were dispersed in $1 \mathrm{ml}$ deionized water by vigorous vortex mixing at temperatures near $55-60^{\circ} \mathrm{C}$, degassed, and 900 $\mu \mathrm{l}$ aliquots were used for gathering DSC thermograms of the main phase transition using a high-high sensitivity multi-cell DSC instrument and $324 \mu \mathrm{l}$ aliquots for the pretransition using a high-sensitivity Nano DSC (both from Calorimetry Sciences Corporation, Lindon, UT), at a scan rate of $10^{\circ} \mathrm{C} / \mathrm{h}$. The quantity of lipid in the DSC samples was progressively increased with additional sterol content to ensure better resolution of the broad low-enthalpy thermotropic transitions, as described earlier (25).

The data were analyzed using Origin 7.5 (OriginLab Corporation, Northampton, MA). The midpoint temperatures, peak widths at half height (inversely related to cooperativity) and areas of the components (proportional to the enthalpy) seen in complex endotherms were estimated with the aid of the Origin non-linear least squares curveand peak-fitting procedures and a custom-coded function based on the assumption that the observed thermogram is a linear combination of components, each of which could be approximated by a reversible two-state transition at thermodynamic equilibrium. The equations used to develop the fitting function are described elsewhere (29).

\section{Results}

The overall pattern of thermotropic phase behaviour observed in sterol/DPPC dispersions

DSC heating scans of DPPC dispersions containing different concentrations of the nine sterols and steroids are shown for comparative purposes in Fig 5 . In the absence of sterols, DPPC heating scans show two sharp endothermic peaks initially centered $\sim 35{ }^{\circ} \mathrm{C}$ and $\sim 41.7{ }^{\circ} \mathrm{C}$, which correspond to the so-called pretransition, a transition from a lamellar gel phase with tilted hydrocarbon chains to a rippled gel phase with tilted hydrocarbon chains ( $\alpha^{\prime}$ '/ $\left.P \alpha^{\prime}\right)$, and a main phase transition from a rippled gel phase to a liquid-crystalline phase $\left(P \alpha^{\prime} / L \alpha\right)$, respectively. From these thermograms, we can measure the phase transition temperature, $\mathrm{T}\left({ }^{\circ} \mathrm{C}\right)$, as a function of sterol concentration. Increases in T reflect the ability of the sterol to stabilize and order the DPPC gel phase. Corresponding measurements of $\Delta \mathrm{T}_{1 / 2}\left({ }^{\circ} \mathrm{C}\right)$, the width of the phase transition at half height, which is inversely related to the cooperativity of the phase transition, measures the ability of the sterol to broaden the phase transition. Increasing the concentration of molecule A (sterol) in molecule B (lipid) typically increases $\Delta T_{1 / 2}$ if the two molecules are miscible. The phase transition enthalpy, $\Delta \mathrm{H}(\mathrm{kcal} / \mathrm{mol})$, is a measure of the energy required for the lipid/sterol mixture to undergo a phase change. The ability of the sterol to abolish the phase transition can be positively correlated to the miscibility of the sterol in the DPPC bilayer. If $\Delta \mathrm{H}=0$, the sterol is essentially fully miscible in the bilayer.

Normally in sterol/DPPC mixtures, increasing the sterol concentration gradually reduces the pretransition temperature $\left(T_{p}\right)$, broadens the pretransition $\left(\Delta T_{p 1 / 2}\right)$, and eventually abolishes the enthalpy $\left(\Delta \mathrm{H}_{\mathrm{p}}\right)$. Similarly, for the main phase transition, increasing the sterol concentration initially produces a multi-component endotherm that consists of a sharp component that is progressively reduced in temperature $\left(T_{m}\right.$ shp $)$, broadened $\left(\Delta T_{1 / 2}\right.$ shp $)$ and reduced in enthalpy $\left(\Delta \mathrm{H}_{\mathrm{m}}\right.$ shp $)$, and a broad component that may either increase (increase stability) or decrease (decrease stability) in temperature $\left(T_{m}\right.$ brd $)$, broaden $\left(\Delta T_{1 / 2}\right.$ brd $)$, and normally initially increase, then decrease in enthalpy $\left(\Delta \mathrm{H}_{\mathrm{m}}\right.$ brd $)$. Overall, with increasing sterol concentration, the sharp component disappears as the broad component grows, and the sum of these two components gives the total enthalpy of the main phase transition $\left(\Delta \mathrm{H}_{\mathrm{m}}\right.$ tot). However, there are important qualitative differences in the effect of sterol ring structure and conformation on the DPPC pretransition and on the two components of the main phase transition, as discussed below.

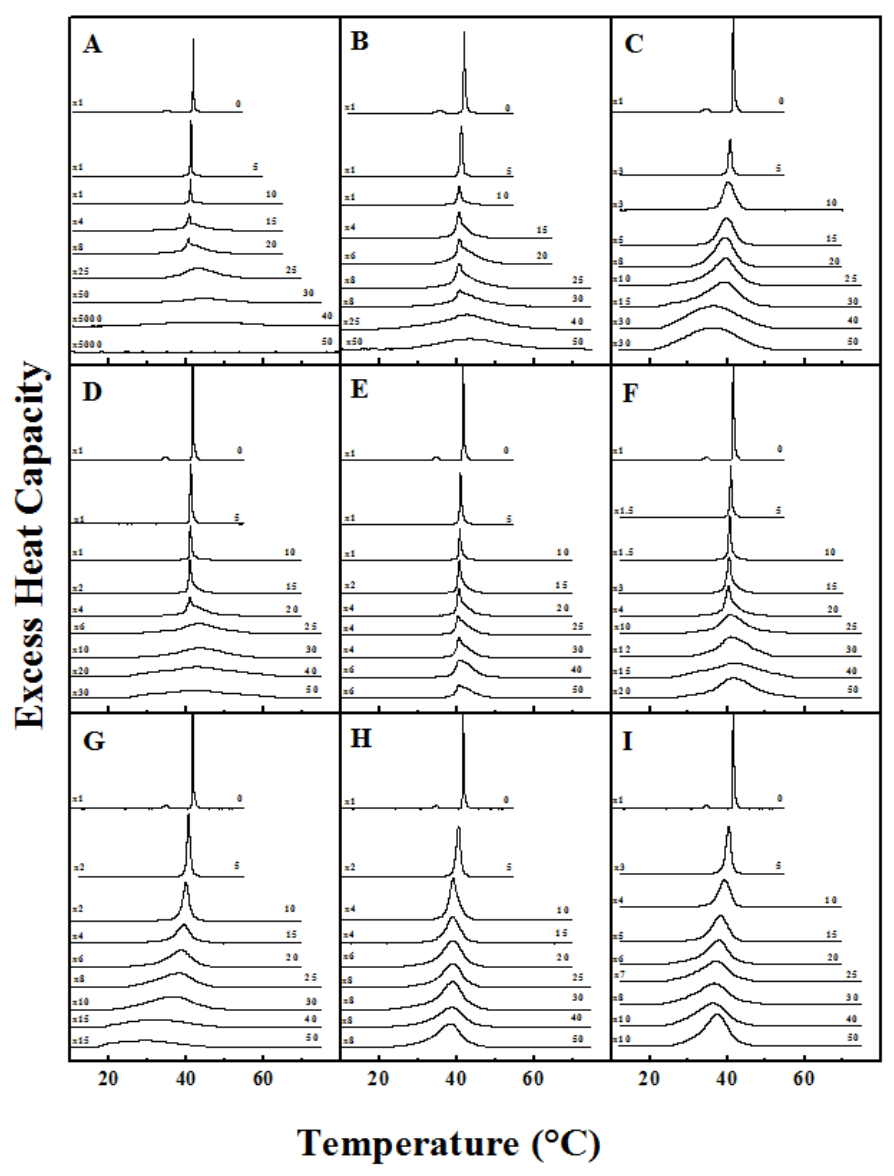

Figure 5. DSC thermograms illustrating the effect of cholesterol1 (A), epicholes-

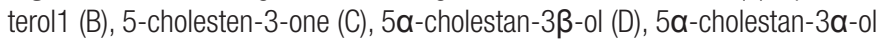
(E), $5 \alpha$-cholestan-3-one (F), $5 \beta$-cholestan- $3 \beta$-ol (G), $5 \beta$-cholestan-3 $\alpha$-ol (H), and $5 \beta$-cholestan-3-one (I) on the gel/liquid-crystalline phase transition of DPPC. The mixtures were heated and cooled at $10^{\circ} \mathrm{C} / \mathrm{h}$ (to maximize resolution and minimize artifacts). The thermograms shown were acquired at the sterol concentrations (mol\%) indicated and have all been normalized against the mass of DPPC used. Y-axis scaling factors are indicated on the left-hand side of each thermogram. ${ }^{1}$ Ref 24. 
The effects of sterols on the pretransition of DPPC

The gradual elimination of the pretransition in the DSC heating scans with increasing sterol concentration are shown in Fig 6 and the derived thermodynamic parameters defined above are shown in Figs 7 (grouped by the functional group at carbon 3) and 8 (grouped by the conformation of carbon 5). All sterol/DPPC mixtures show a reduction in $T_{p}$ with increasing sterol concentration (Figs 7,8 A-C), but mixtures containing the steroids are more effective at destabilizing the La' phase and at perturbing the phase transition than the corresponding sterols. A notable exception to this trend is $5 \alpha-\mathrm{H}, 3-\mathrm{K}$, where $T_{p}$ levels off at $\sim 32{ }^{\circ} \mathrm{C}$. The $3 \alpha$-ols destabilize the $L \alpha$ ' phase less than $3 \alpha$-ols, with the largest differences of $\sim 2{ }^{\circ} \mathrm{C}$ within the C5,6 double bond group (3a-ol, 3a-ol, 3-K), but shows almost perfect overlap within the $5 \alpha-\mathrm{H}$ group (3a-ol, $3 \alpha-o l, 3-K) . \Delta T_{p^{1 / 2} / 2}$ increases most rapidly for EChol and Chol, demonstrating their ability to better disorder the pretransition (Figs 7D, 7E, 8D). The $5 \alpha-\mathrm{H}$ sterols have a similar effect on the DPPC transition, although the $5 \alpha-\mathrm{H}, 3 \alpha-$ ol/5a-H,3a-ol pair show fewer differences between each other than those of the EChol/Chol pair (Figs 8D \& E). When the data from the three ketones are compared $(5 \alpha-\mathrm{H}, 5 \beta-$ $\mathrm{H}, \mathrm{C5}, 6$ double bond), the curves reach a maximum at

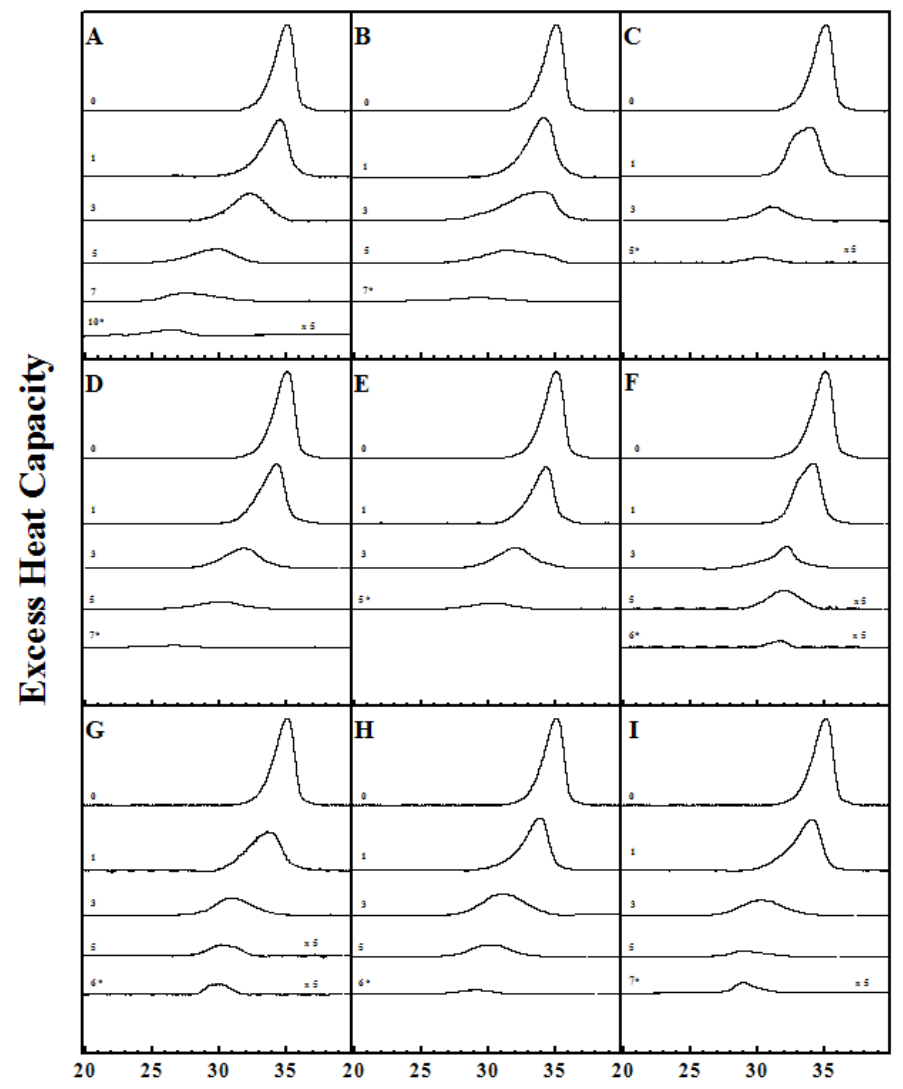

Temperature $\left({ }^{\circ} \mathrm{C}\right)$

Figure 6. DSC thermograms illustrating the effect of cholesterol1 (A), epicholes-

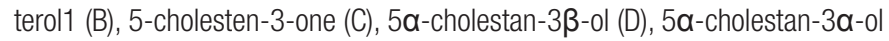

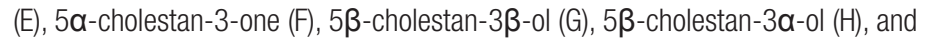
$5 \beta$-cholestan-3-one (I) on the pre-transition of DPPC. The mixtures were heated and cooled at $10^{\circ} \mathrm{C} / \mathrm{h}$ (to maximize resolution and minimize artifacts). The thermograms shown were acquired at the sterol concentrations (mol\%) indicated on the panels left side and have all been normalized against the mass of DDPC used. Y-axis scaling factors are indicated on the right-hand side of each thermogram. The * indicate the highest mol\% where the pre-transition was observed. ${ }^{1}$ Ref24.

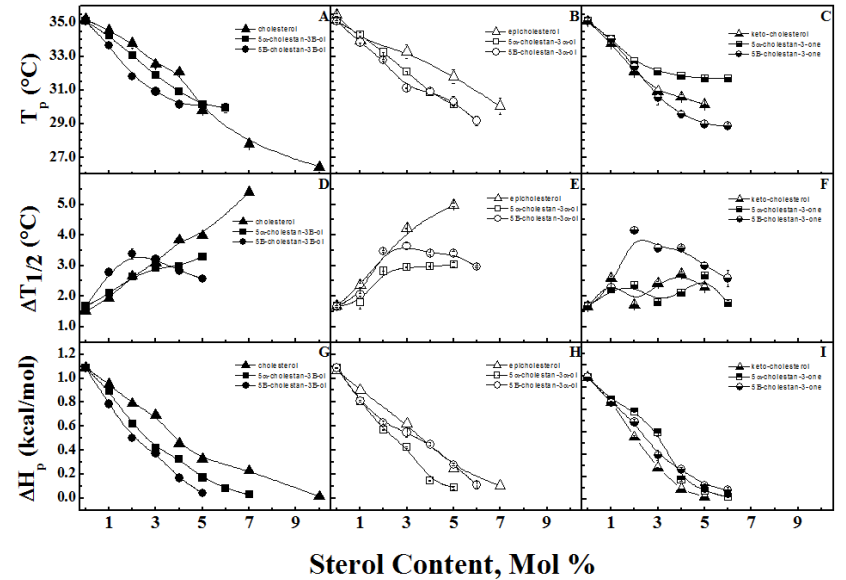

Figure 7. The effect of increases in sterol concentration on $T p(A-C), \triangle T 1 / 2$ (D-F), and $\triangle H p(G-I)$ on the pretransition of DPPC. These parameters are grouped by $\mathrm{C}-3$ functional group. The left column is the $\mathrm{C}-3 \beta$-hydroxyl sterols, the middle column is the $\mathrm{C}$-3a-hydroxyl sterols, and the right column is the C-3-ketosteroids. When a standard error bar is not visible, it is less than or equal to the symbol dimensions. Data for cholesterol and epicholesterol is taken from Ref 24.

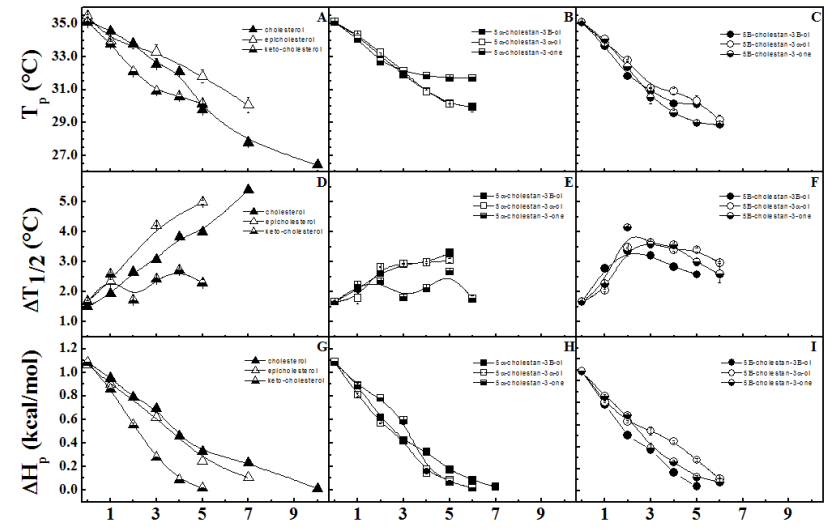

Sterol Content, Mol \%

Figure 8. The effect of increasing sterol concentration on Tp (A-C), $\triangle T 1 / 2$ (D-F), and $\triangle \mathrm{Hp}(\mathrm{G}-\mathrm{I})$ on the pretransition of DPPC. These parameters are grouped by C-5 functional group. The left column is C-5,6-double-bond sterols, the middle column is $5 \alpha$-sterols, and the right column is $5 \beta$-sterols. When a standard error bar is not visible, it is less than or equal to the symbol dimensions. Data for cholesterol and epicholesterol is taken from Ref 24.

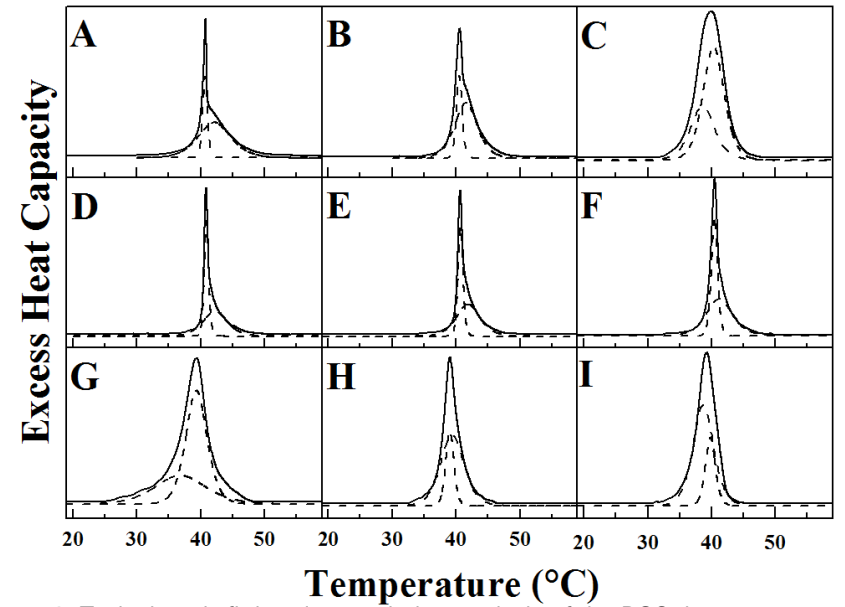

Figure 9. Typical peak-fitting deconvolution analysis of the DSC thermograms exhibited by $15 \mathrm{~mol} \%$ cholesterol1 (A), $15 \mathrm{~mol} \%$ epicholesterol1 (B), $15 \mathrm{~mol} \%$

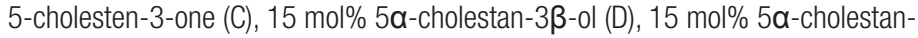

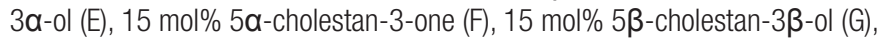
$10 \mathrm{~mol} \% 5 \beta$-cholestan- $3 \alpha-0 l(H)$, and $10 \mathrm{~mol} \% 5 \beta$-cholestan-3-one/DPPC (I) mixtures heated and cooled at $10^{\circ} \mathrm{C} / \mathrm{h}$. To facilitate visibility, the filled curves are slightly displaced along the $y$-axis. The analysis is performed using a custom coded algorithm for a first-order phase transition. ${ }^{1}$ Ref 24. 
$\sim 3 \mathrm{~mol} \%$ and then decrease (Fig 7F). Similar behaviour is observed in a comparison of the $\Delta T_{p 1 / 2}$ values for $5 \alpha-H(3 \alpha-$ ol, $3 \alpha-o l, 3-K)$, but the result is less consistent and shows more scatter (Fig 8E). In the case of $5 \alpha-\mathrm{H}$ (3a-ol, 3a-ol, 3-K), the curves smoothly and consistently achieve a maximum $\Delta \mathrm{T}_{\mathrm{p} / 2}$ of $\sim 3-4{ }^{\circ} \mathrm{C}$ before steadily decreasing to $\sim 2{ }^{\circ} \mathrm{C}$ (Fig $8 \mathrm{~F})$, suggesting the existence of at least two components in their pretransition endotherms. The significance of this observation is discussed later.

All sterols and steroids are more effective in decreasing $\Delta \mathrm{H}_{\mathrm{p}}$ than Chol (Fig 7,8 G-I), abolishing the pretransition by $\sim 5-7 \mathrm{~mol} \%$, whereas it persists up to $\sim 10 \mathrm{~mol} \%$ in the Chol/DPPC mixtures. This indicates that these sterols and steroids occupy a larger cross-sectional area than Chol and/or disorder the hydrocarbon chains of adjacent DPPC molecules to a greater extent. This allows relief of the area mismatch between the relatively larger PC polar headgroups and the relatively smaller hydrocarbon chains, such that energetically unfavorable chain tilting is not required at lower sterol concentrations. Among all of the $3 \alpha$-ols $(5 \alpha-\mathrm{H}, 5 \alpha-\mathrm{H}, \mathrm{C} 5,6$ double bond), the $5 \alpha-\mathrm{H}$ sterol more effectively abolishes the pretransition than the $5 \mathrm{a}-\mathrm{H}$ sterol ( $5 \mathrm{~mol} \%$ versus $7 \mathrm{~mol} \%$; Fig $7 \mathrm{G}$ ), In contrast, with the $3 \alpha$-ols $(5 \alpha-H, 5 \alpha-H, C 5,6$ double bond), the $5 \alpha-H$ sterol more effectively abolishes the pretransition than the $5 \alpha-\mathrm{H}$ sterol (Fig $7 \mathrm{H})$. The trends change for the steroids, with CholK more effectively abolishing the pretransition, and $5 \alpha-\mathrm{H}, 3-\mathrm{K}$ being the least effective below $4 \mathrm{~mol} \%$, and $5 \alpha-\mathrm{H}, 3-\mathrm{K}$ being the most effective at higher concentration (Fig 7I). A comparison based on the C5,6-conformation shows that within this group, CholK more effectively abolishes the pretransition than either Chol or EChol, with EChol being marginally better at reducing the enthalpy than Chol (Fig 8G). The plots show more overlap for the $5 a-H$ and $5 \alpha-\mathrm{H}$ groups, with $5 \alpha-\mathrm{H}, 3-\mathrm{K}$ being the least disruptive below $3 \mathrm{~mol} \%$, but $5 \mathrm{a}-\mathrm{H}, 3 \mathrm{a}-\mathrm{Ol}$ and $5 \mathrm{a}-\mathrm{H}, 3-\mathrm{K}$ appear to be equally more disruptive than $5 \mathrm{a}-\mathrm{H}, 3 \mathrm{a}-\mathrm{Ol}$ at higher sterol concentrations (Fig $8 \mathrm{H}$ ). For the $5 \alpha-\mathrm{H}$ group, the $3 \alpha-\mathrm{ol}$ most effective abolishes the pretransition, whereas like $5 \alpha-\mathrm{H}, 3-\mathrm{K}$, $5 \beta-\mathrm{H}, 3-\mathrm{K}$ is initially the least disruptive, but becomes more effective than the $5 \alpha-\mathrm{H}, 3 \alpha-\mathrm{ol}$ above 2 mol\% (Fig 8I).

\section{The effects of sterol concentration on the main phase} transition of DPPC

The DSC data shown in Fig 5 indicates that at low to moderate sterol concentrations, all sterol-containing DPPC bilayers exhibit asymmetric thermograms for the main $\mathrm{P}_{\mathrm{b}}{ }^{1} /$ $L_{a}$ phase transition, which consists of overlapping sharp and broad thermal events representing the melting of sterol-poor and sterol-rich domains respectively (Fig 9). For the deconvolved sterol-poor component of DPPC/ Chol mixtures, $T_{m}$ shp decreases (destabilization of the $\mathrm{Pa}$ ' phase), $\Delta \mathrm{T}_{1 / 2} \operatorname{sh}^{\mathrm{m}}$ increases (perturbation of the phase transition of the sterol-poor domain), and $\Delta \mathrm{H}_{\mathrm{m}}$ shp decreases (abolishing the sterol-poor domain phase transition). The broad component shows a more complex dependence on sterol content and, in most cases, is the only component persisting at higher sterol concentrations. Perhaps the most significant observations are that for DPPC/Chol mixtures: $\mathrm{T}_{\mathrm{m}}$ brd continues to increase at high sterol concentrations, signifying a stabilization of the gel state. Concurrently, the $\Delta \mathrm{T}_{1 / 2}$ brd increases significantly above $30 \mathrm{~mol} \%$ sterol and the $\Delta \mathrm{H}_{\mathrm{m}}$ brd is completely abolished, indicating that additional Chol enters the DPPC bilayer and that it is fully miscible with DPPC at higher concentrations. This forms a stable and ordered lamellar phase. However, there are significant differences in the trends observed in the thermodynamic parameters obtained from binary lipid/sterol mixtures with changes in sterol structure (23-25) indicating the subtle influences of sterol structure on the DPPC gel and liquidcrystalline bilayer properties, which have been largely ignored in favor of the myth that Chol possess a rigid ring system with a flexible but fully extended side chain. In the sections below, a detailed report is provided about the effects of changes in sterol ring chemical structure on the sharp and broad components of the DPPC main phase transition and their potential consequences on lateral phase separation in lipid rafts.

\section{The effects of sterols on the sharp component of the} DPPC main phase transition

Panels A of Figs 10-12 shows a gradual decrease in $T_{m}$ shp with increasing sterol concentrations for each sterol/ DPPC system. Among the $3 \beta$-ols, $5 \beta-\mathrm{H}, 3 \beta$-ol shows the greatest decrease in $T_{m}$ shp and, thus, the greatest gel phase destabilization. In contrast, $5 \alpha-\mathrm{H}, 3 \beta$-ol and Chol show similar curves in which there is only moderate destabilization (Fig $10 A)$. Whereas The $\Delta T 1 / 2^{\text {shp }}$ of $5 \beta-H, 3 \beta$-ol broadens rapidly up to $15 \mathrm{~mol} \%$, those of $\mathrm{Chol}$ and $5 \alpha-\mathrm{H}, 3 \beta$-ol increase more slowly and persist up to $20 \mathrm{~mol} \%$ sterol (Fig 10B). On the other hand, there is not much difference in the $\Delta \mathrm{H}_{\mathrm{m}}$ shp values for the $3 \beta$-ols, where the transition is abolished between $15 \mathrm{~mol} \%$ for the $5 \beta-\mathrm{H}, 3 \beta-\mathrm{ol}$ and $20 \mathrm{~mol} \%$ for both Chol and $5 \alpha-\mathrm{H}, 3 \beta-\mathrm{OH}$ (Fig $10 \mathrm{C}$ ). This suggests that C5-conformation changes do not significantly alter the ability of the $3 \beta$-ols to abolish the transition in the sterolpoor domains. Among all of the $3 \alpha-\mathrm{ols}, 5 \beta-\mathrm{H}, 3 \alpha-\mathrm{ol}$ shows the greatest decrease in $T_{m}$ shp , but the sharp component of EChol and $5 \alpha-\mathrm{H}, 3 \beta-\mathrm{ol}$ level off at $10-15 \mathrm{~mol} \%$ sterol and persist until $40 \mathrm{~mol} \%$ and $50 \mathrm{~mol} \%$ sterol, respectively. This may indicate a difference in the sterol concentrationdependent partitioning between the sterol-poor and sterolrich domains (Fig 11A). Corresponding $\Delta \mathrm{T}_{1 / 2}$ shp values show that $5 \beta-\mathrm{H}, 3 \alpha-\mathrm{ol}$ has the greatest ability to broaden the main transition sharp component at low sterol concentrations, while EChol and $5 \alpha-\mathrm{H}, 3 \alpha-\mathrm{ol}$ are more effective at higher sterol concentrations (Fig 11B). Up to $\sim 7 \mathrm{~mol} \%$, all three $3 \alpha$-ols $(5 \alpha-\mathrm{H}, 5 \alpha-\mathrm{H}, \mathrm{C} 5,6$ double bond) show the same negative enthalpy coefficients. EChol though does not abolish the sharp component by 40 mol\% and $5 \alpha-\mathrm{H}, 3 \alpha-\mathrm{ol}$ is the least effective overall, showing a value of $\sim 1 \mathrm{kcal} /$ $\mathrm{mol}$ at $50 \mathrm{~mol} \%$ sterol (Fig $11 \mathrm{C})$. The steroids $(5 \alpha-\mathrm{H}, 5 \alpha-$ $\mathrm{H}, \mathrm{C} 5,6$ double bond) are similar in the initial decrease of $\Delta T_{1 / 2}^{\text {shp }}$, indicating that they destabilize the $\mathrm{P}_{\mathrm{b}^{\prime}}$ phase to the same extent (Fig 12A). $5 \beta-\mathrm{H}, 3-\mathrm{K}$ is marginally better at broadening the main transition below 7 mol\% steroid than CholK. Above $7 \mathrm{~mol} \%$ steroid, CholK significantly broadens the gel/liquid crystalline phase transition of the sterol-poor region, whereas $5 \alpha-\mathrm{H}, 3-\mathrm{K}$ is only moderately effective. (Fig 12B). The steroids abolish the sharp component of the 


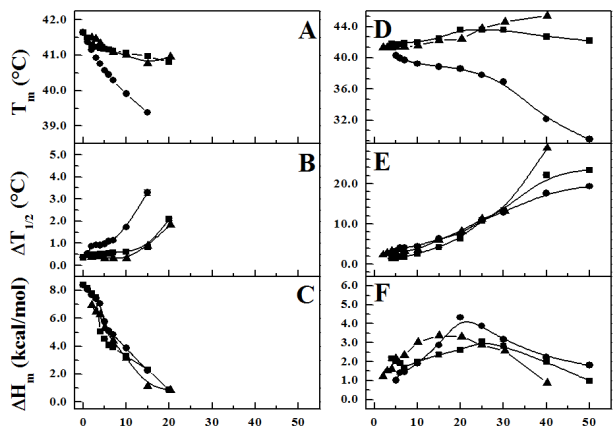

Sterol Content, Mol\%

Figure 10. The thermodynamic parameters of $3 \beta-\mathrm{OH}$ sterols for the deconvolved sharp $(\mathrm{A}-\mathrm{C})$ and broad (D-F) components obtained from DSC heating thermograms of cholesterol ${ }^{1}(\mathbf{\Lambda}), 5 \alpha$-cholestan-3 $\beta$ $\mathrm{Ol}(\boldsymbol{\square})$, and $5 \beta$-cholestan-3 $\beta$-ol (-)/DPPC mixtures as a function of sterol concentration. The error bars were typically equal to or smaller than the size of the symbols. ${ }^{1}$ Ref 24.
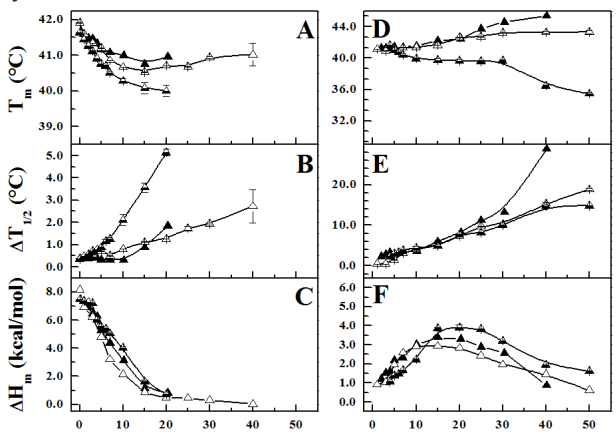

Sterol Content, Mol\%

Figure 13. The thermodynamic parameters of $\mathrm{C}-5,6$ double-bond sterols for the deconvolved sharp (A-C) and broad (D-F) components obtained from DSC heating thermograms of cholesterol1 $(\mathbf{\Delta})$, epicholesterol1 $(\triangle)$, and keto-cholesterol $(\boldsymbol{\Delta}) / \mathrm{DPPC}$ mixtures as a function of sterol concentration. The error bars were typically equal to or smaller than the size of the symbols. ${ }^{1}$ Ref 24.

main transition fairly similarly with $5 \beta-\mathrm{H}, 3-\mathrm{K}$ being the best, disappearing at $10 \mathrm{~mol} \%$, whereas $5 \alpha-\mathrm{H}, 3-\mathrm{K}$ persists to 25 mol\% (Fig 12C).

Figs $13-15$ re-plot the data from Figs $10-12$ by C5-conformation. Generally speaking, for the sharp component, steroids ( $5 \alpha-\mathrm{H}, 5 \alpha-\mathrm{H}, \mathrm{C} 5,6$ double bond) are the best able to destabilize the $\mathrm{P}_{\mathrm{b}^{\prime}}$ phase and the $3 \beta$-ols (5a-H, 5a-H, C5,6 double bond) show the least ability (Figs 13-15 A). The C5,6 double bond group (3a-ol, 3a-ol, 3-K) shows the greatest spread of $\mathrm{T}_{\mathrm{m}}$ shp curves, but the $5 \beta-\mathrm{H}$ group $(3 \alpha-o l, 3 \alpha-o l, 3-K)$ has the greatest overlap over the entire range of concentrations (Fig 13A, 15A). There is variance among the $\Delta T_{1 / 2}$ shp curves based on the grouping by $\mathrm{C} 5$-conformation, but overall, the steroids $(5 \alpha-\mathrm{H}, 5 \alpha-$ $\mathrm{H}, \mathrm{C} 5,6$ double bond) broaden the main transition more quickly at low concentrations than the sterols $(5 \alpha-\mathrm{H}, 5 \alpha-\mathrm{H}$, C5,6 double bond) (Fig 13-15 B). Unlike the $5 \alpha-\mathrm{H}$ sterols (3a-ol, $3 \alpha-\mathrm{ol})$ and $\mathrm{C} 5,6$ double bond sterols (3a-ol, $3 \alpha-\mathrm{ol})$, the $5 \beta-\mathrm{H}$ sterols ( $3 \alpha-\mathrm{ol}, 3 \alpha-\mathrm{ol})$ abolish the sharp component completely by $10-15 \mathrm{~mol} \%$ (Fig $15 \mathrm{C}$ ), whereas $5 \alpha-\mathrm{H}, 3 \alpha-$ ol and EChol do not readily abolish the sharp transition (Fig 13C, 14C). This suggests that sterol concentration-
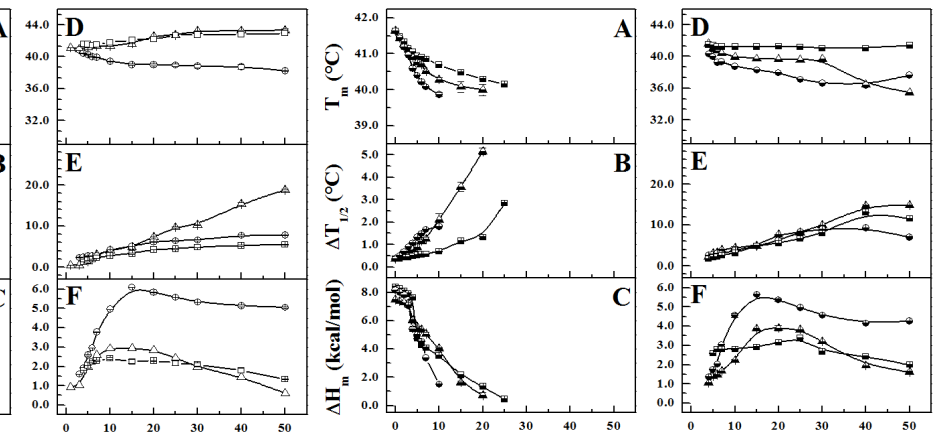

Sterol Content, Mol\%

Figure 12. The thermodynamic parameters of $\mathrm{C}$ 3-keto-sterol for the deconvolved sharp (A-C) and broad (D-F) components obtained from DSC heating thermograms of 5-cholesten-3-one $(\boldsymbol{\Delta}), 5 \boldsymbol{\alpha}$-cholestan3-one $(\boldsymbol{\theta})$, and $5 \beta$-cholestan-3-one $(\boldsymbol{\theta}) / \mathrm{DPPC}$ mixtures as a function of sterol concentration. The error bars were typically equal to or smaller than the size of the symbols.
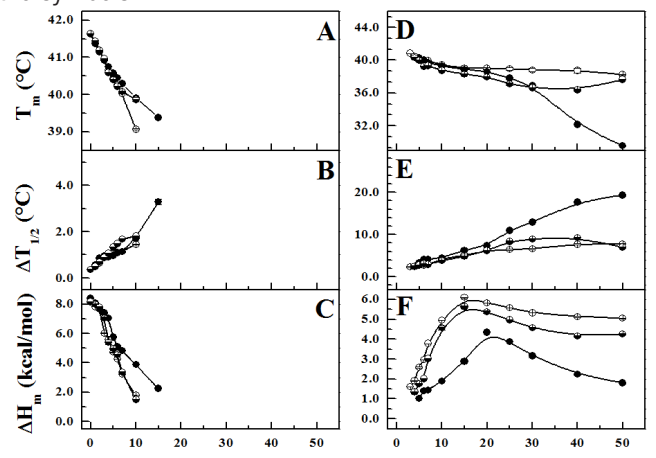

Sterol Content, Mol\%

Figure 15. The thermodynamic parameters of $5 \beta-H$ sterols for the deconvolved sharp (A-C) and broad (D-F) components obtained from DSC heating thermograms

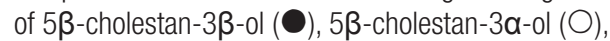
and $5 \beta$-cholestan-3-one ( $(-) / D P P C$ mixtures as a function of sterol concentration. The error bars were typically equal to or smaller than the size of the symbols. dependent partitioning between sterol-poor and sterol-rich domains is more definitive for the $5 \beta-\mathrm{H}$ sterols ( $3 \alpha-\mathrm{ol}, 3 \alpha-$ ol) on account of the $5 \beta-\mathrm{H}$ distorted conformation.

The effects of sterols on the broad component of the DPPC main phase transition

Among all the sterols, Chol is the most effective at stabilizing the $\mathrm{Pa}^{\prime}$ phase in the sterol-rich gel state showing a net temperature shift of $\sim+2.5^{\circ} \mathrm{C}$ by $40 \mathrm{~mol} \%$. However, $5 \beta-\mathrm{H}, 3 \beta-\mathrm{ol}$ is the most destabilizing, displaying a $-12{ }^{\circ} \mathrm{C}$ shift at $50 \mathrm{~mol} \%$ sterol. The $5 \alpha-\mathrm{H}, 3 \beta$-ol shows intermediate behaviour, increasing to $\sim+2{ }^{\circ} \mathrm{C}$ by $20 \mathrm{~mol} \%$ before coming back to $\sim 0{ }^{\circ} \mathrm{C}$ net temperature shift at 50 mol\% concentration (Fig 10D). Whereas the $5 \beta-\mathrm{H}, 3 \beta-\mathrm{ol}$ is the least able to broaden the main transition among the $3 \beta$-ols, the $\Delta T_{1 / 2}$ brd rapidly increases for Chol at 30-40 mol\% sterol (Fig 10E). For the $\Delta \mathrm{H}_{\mathrm{m}}$ brd curves, peak maxima occur at about $15 \%$ for Chol, $20 \%$ for $5 \beta-\mathrm{H}, 3 \beta$-ol, and $25 \%$ for $5 \alpha-\mathrm{H}, 3 \beta-$ ol. $5 \beta-\mathrm{H}, 3 \beta-\mathrm{ol}$ is the least able to abolish $\Delta \mathrm{H}_{\mathrm{m}}$ brd, whereas Chol is the most effective (Fig 10F).

Similar trends are observed for the $3 \alpha-0$ ls $(5 \alpha-\mathrm{H}, 5 \alpha-$ $\mathrm{H}, \mathrm{C} 5,6$ double bond), where EChol and $5 \alpha-\mathrm{H}, 3 \alpha-\mathrm{ol}$ show a small increase in $\mathrm{T}_{\mathrm{m}}$ brd and thus a reduced ability to 
stabilize the gel state compared to Chol. While $5 \beta-\mathrm{H}, 3 \alpha-$ ol has only a slightly negative $\mathrm{T}_{\mathrm{m}}$ brd curve when compared to $T_{m}$ brd for $5 \beta-H, 3 \beta-o l$ (Fig 11D), the broadening of the phase transition follows the same pattern as that seen in the $3 \beta$-ols $(5 \alpha-\mathrm{H}, 5 \alpha-\mathrm{H}, \mathrm{C} 5,6$ double bond) even though $\Delta \mathrm{T}_{1 / 2}$ brd only increases to $\sim 18{ }^{\circ} \mathrm{C}$ in $50 \mathrm{~mol} \%$ EChol (Fig $11 \mathrm{E})$. Although similar decreasing trends occur in the $\Delta \mathrm{H}_{\mathrm{m}}$ brd of the $3 \alpha$-ols compared to the $3 \beta$-ols $(5 \alpha-\mathrm{H}, 5 \alpha-\mathrm{H}$, $\mathrm{C} 5,6$ double bond), there are significant differences in that $5 \beta-\mathrm{H}, 3 \alpha$-ol reaches a peak maximum $\Delta \mathrm{H}_{\mathrm{m}}$ brd value of about $\sim 6 \mathrm{kcal} / \mathrm{mol}$ at $15 \mathrm{~mol} \%$ and levels off to only $\sim 5.5 \mathrm{kcal} /$ mol by $50 \mathrm{~mol} \%$. Up to $30 \mathrm{~mol} \%$ sterol, the $\Delta \mathrm{H}_{\mathrm{m}}$ brd curve for $5 \mathrm{a}-\mathrm{H}, 3 \mathrm{a}-\mathrm{ol}$ lies below that of EChol before switching positions. Both of these sterols are less able to abolish the phase transition relative to their $3 \beta$-ol counterparts, but the difference is less than that observed for the corresponding $\Delta \mathrm{H}_{\mathrm{m}}{ }^{\text {brd }}$ values of the $5 \alpha-\mathrm{H}$ sterols (3a-ol, 3a-ol) (Fig 11F). The steroids $(5 \alpha-\mathrm{H}, 5 \alpha-\mathrm{H}, \mathrm{C} 5,6$ double bond) behave very similarly to their $3 \alpha-$ ol counterparts in terms of their ability to cooperate and their ability to abolish the phase transition (Fig 12E and $F$, respectively), although the $\Delta T_{1 / 2}$ brd curves either plateau or decrease slightly by $\sim 2-3{ }^{\circ} \mathrm{C}$ at $50 \mathrm{~mol} \%$ for all steroids. There are also differences in the $T_{m}$ brd curves for the steroids ( $5 \alpha-\mathrm{H}, 5 \alpha-\mathrm{H}, \mathrm{C} 5,6$ double bond). The $5 \alpha-\mathrm{H}$ steroid shows the smallest change in $T_{m}$ brd (Fig 12D) and thus gel-state stabilization, being essentially constant at all sterol concentrations. In contrast, CholK shows a decrease of $\sim{ }^{\circ} \mathrm{C}$ by $15 \mathrm{~mol} \%$, holding steady at around $40^{\circ} \mathrm{C}$ to 30 mol\% steroid followed by a linear decrease of an additional $4{ }^{\circ} \mathrm{C}$ by $50 \mathrm{~mol} \%$ steroid indicating a decrease in gel-state destabilization. $5 \beta-\mathrm{H}, 3-\mathrm{K}$ destabilizes the gel-state bilayer the most overall. However, the curve is concave above $25 \mathrm{~mol} \%$ (Fig 12D), consistent with the appearance of additional endotherms in the heating thermograms below and above the main transition centered at $\sim 22{ }^{\circ} \mathrm{C}$ and $52-58{ }^{\circ} \mathrm{C}$, respectively (Fig 16 ). In a similar fashion, panel $D$ of Figs $13-15$ shows that the steroids more effectively destabilize the $P_{b}$ ' phase, whereas the $3 \beta$-ols are the least effective, with the exceptions of $5 \alpha-\mathrm{H}, 3 \alpha-\mathrm{ol}$ being more

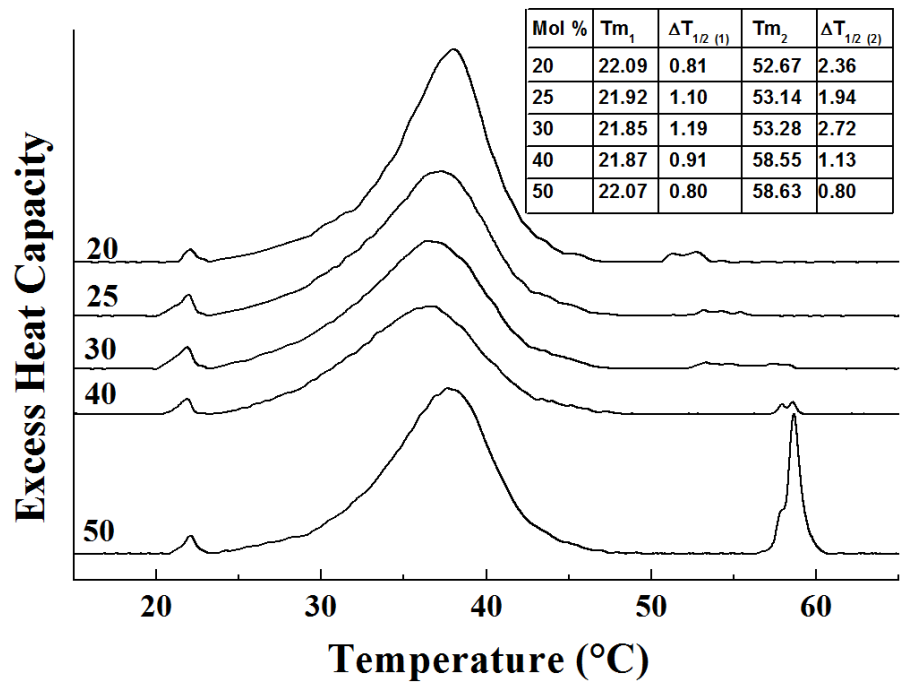

Figure 16. Mass normalized DSC thermograms for $5 \beta$-cholestan-3-one/DPPC mixtures heated and cooled at $10^{\circ} \mathrm{C} / \mathrm{h}$ (to maximize resolution and minimize artifacts) illustrating sterol crystallite formation above and below and main chain-melting transition. The transition temperature and the half-widths of the endotherms are indicated on the graph. stabilizing above 40 mol\% than $5 \alpha-\mathrm{H}, 3 \beta-o l$ (Fig 14D) and $5 \beta-\mathrm{H}, 3 \beta-\mathrm{ol}$, which was the most effective at destabilizing the DPPC gel state at high sterol concentrations (Fig 15D).

The effects of sterols on the total enthalpy component and net temperature shifts of the DPPC main phase transition

Fig $17(\mathrm{~A}-\mathrm{C})$ plots the $\Delta \mathrm{H}_{\mathrm{m}}{ }^{\text {tot }}$ of all the sterol/DPPC systems. At less than $10 \mathrm{~mol} \%$ sterol, Chol is the least effective of the $3 \beta$-ols in reducing $\Delta \mathrm{H}_{\mathrm{m}}$ tot. At all sterol concentrations $5 \alpha-\mathrm{H}, 3 \beta-$ ol more effectively reduces $\Delta \mathrm{H}_{\mathrm{m}}$ tot than $5 \beta-\mathrm{H}, 3 \beta-$ ol, although above $30 \mathrm{~mol} \%$, Chol is the most effective $3 \beta$-ol (Fig 17A). The trends are more straightforward for the $3 \alpha$-ols, with $5 \beta-\mathrm{H}, 3 \alpha-\mathrm{ol}$ being the least effective sterol in reducing $\Delta \mathrm{H}_{\mathrm{m}}$ tot followed by $5 \alpha-\mathrm{H}, 3 \alpha-\mathrm{Ol}$ and EChol (Fig 17B). The behaviour is different again for the steroids, with $5 \beta-\mathrm{H}, 3-\mathrm{K}$ being the least effective in reducing the enthalpy at higher sterol concentrations, just like the $5 \beta-\mathrm{H}$ sterols ( $3 \alpha-$ ol, 3a-ol). However, below $10 \mathrm{~mol} \%, 5 \beta-\mathrm{H}, 3-\mathrm{K}$ is best able to reduce $\Delta \mathrm{H}_{\mathrm{m}}$ tot intersecting with the curves of the other two steroids at $\sim 10-20 \mathrm{~mol} \%$. From $15-40 \mathrm{~mol} \%, 5 \mathrm{a}-\mathrm{H}, 3-\mathrm{K}$ most effectively reduces $\Delta \mathrm{H}_{\mathrm{m}}$ tot whereas above $40 \mathrm{~mol} \%$, CholK is the most effective, as was evident with Chol and EChol (Fig 17C). This suggests that the C5,6 double bond plays an important role in favorable lipid/sterol interactions while reducing $\Delta \mathrm{H}_{\mathrm{m}}$ at high sterol concentrations. When

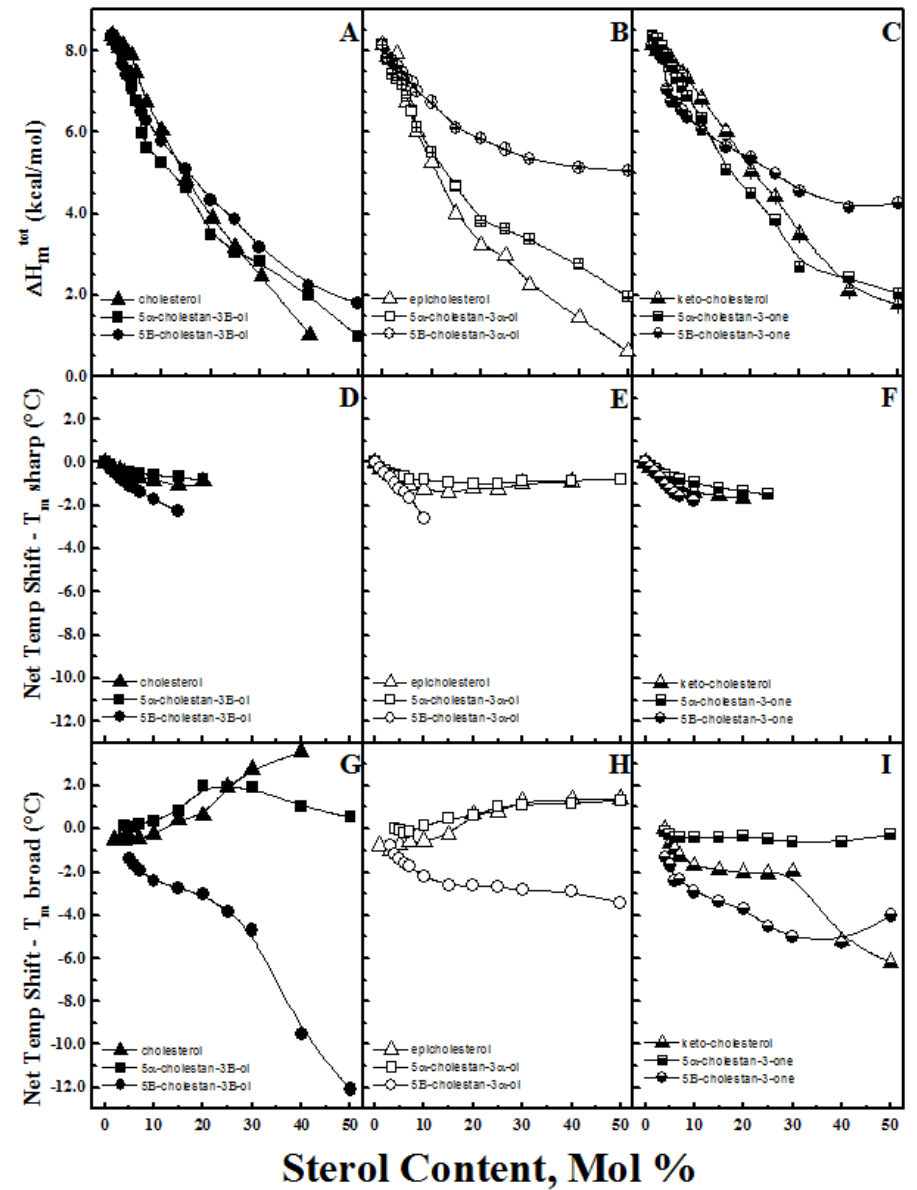

Figure 17. Total overall enthalpy $(A, B, C ; \triangle H m)$ and net temperature shifts for the sharp $\left(\mathrm{D}, \mathrm{E}, \mathrm{F} ;{ }^{\circ} \mathrm{C}\right)$ and broad $\left(\mathrm{G}, \mathrm{H}, \mathrm{l} ;{ }^{\circ} \mathrm{C}\right)$ components of the sterol/DPPC binary mixtures studied, grouped by $\mathrm{C}-3$ functional group. The left column is $\mathrm{C}-3 \beta$-hydroxyl sterols, the middle column is $\mathbf{C}-3 \boldsymbol{\alpha}$-hydroxyl sterols, and the right column is C-3-keto-sterols. When a standard error bar is not visible, it is less than or equal to the symbol dimensions. Data for cholesterol and epicholesterol is taken from Ref 24. 


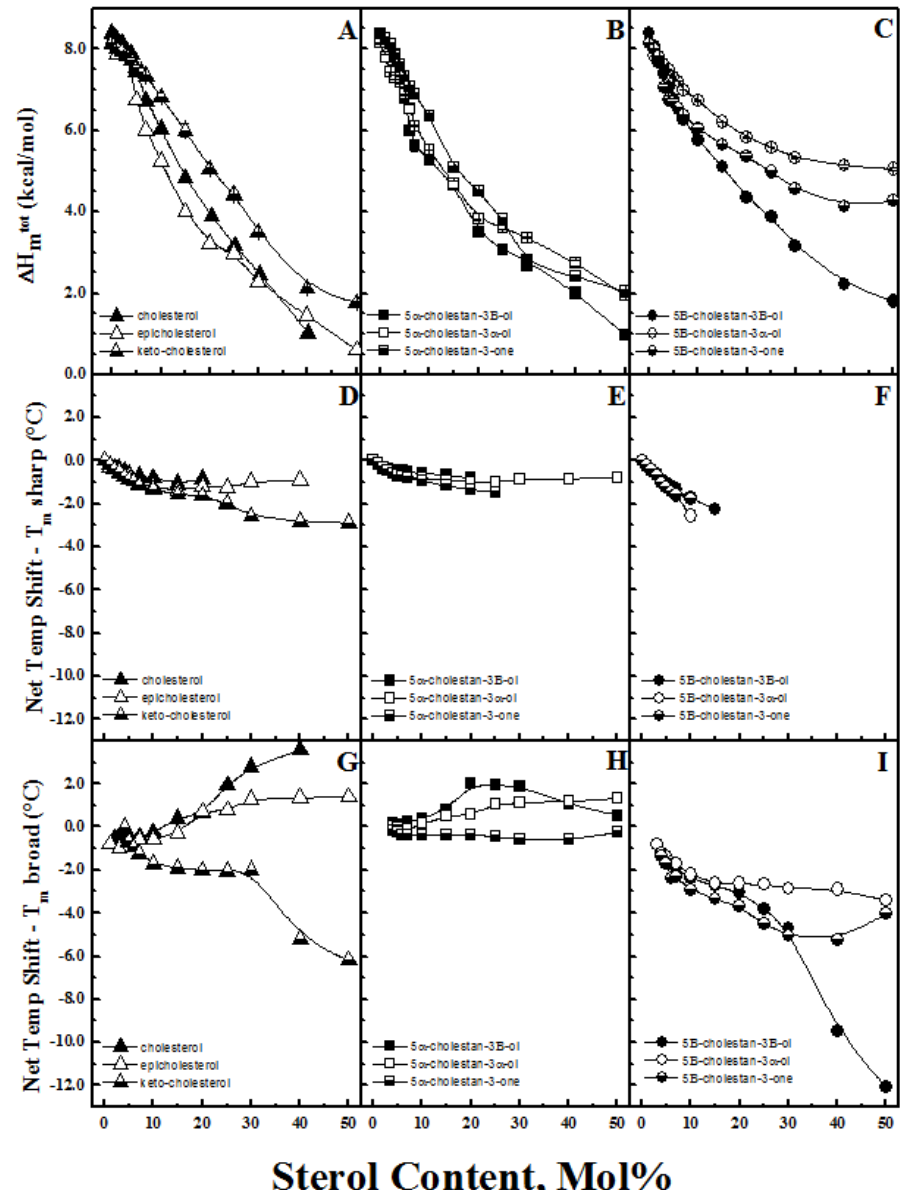

Figure 18. Total overall enthalpy (A, B, C; $\triangle H m)$ and net temperature shifts for the sharp $\left(\mathrm{D}, \mathrm{E}, \mathrm{F} ;{ }^{\circ} \mathrm{C}\right)$ and broad $\left(\mathrm{G}, \mathrm{H}, \mathrm{I} ;{ }^{\circ} \mathrm{C}\right)$ components of the sterol/DPPC binary mixtures grouped by $\mathrm{C}-5$ conformation. The left column is $\mathrm{C}-5,6$ doublebond sterols, the middle column is $5 \alpha-\mathrm{H}$ sterols, and the right column is $5 \beta-\mathrm{H}$ sterols. When a standard error bar is not visible, it is less than or equal to the symbol dimensions. Data for cholesterol and epicholesterol is taken from Ref 24.

grouped by C5-conformation, CholK reduces $\Delta \mathrm{H}_{\mathrm{m}}^{\text {tot }}$ the least, and $\mathrm{EChol}$ is better than Chol to reduce enthalpy until about 40 mol\% (Fig 18A). For the $5 \alpha-\mathrm{H}$ and $5 \beta-\mathrm{H}$ groups, the $3 \beta$-ols are the most effective at reducing $\Delta \mathrm{H}_{\mathrm{m}}$ tot at all sterol concentrations, whereas above $30 \mathrm{~mol} \%$ the $3 a$-ols are less effective than the steroids. Below 30 mol\% for the $5 \alpha-\mathrm{H}$ group, the steroid was the least effective at $\Delta \mathrm{H}_{\mathrm{m}}$ tot reduction, while $5 \beta-H, 3 \alpha$-ol was the least effective of all the sterols and studies to reduce $\Delta \mathrm{H}_{\mathrm{m}}$ tot at all concentrations (Fig 18B,C). An interpretation of this observation will follow in the Discussion.

A plot of the net temperature shift for both the sharp and broad components of all sterols are shown in Figs 17$18 \mathrm{D}-\mathrm{F}$ and G-I, respectively. The net temperature shift is defined as the temperature difference between the $T_{m}$ of pure DPPC and the $T_{m}$ shp or $T_{m}$ brd measured from the DSC endotherms. Grouped by $\mathrm{C} 3$ functionality, it is evident that the $5 \mathrm{a}-\mathrm{H}$ sterols are more perturbing than their $5 \mathrm{a}-\mathrm{H}$ and C5,6-double bond counterparts for both the sharp and board components (Fig 17 D-I). However, when grouped by C5 conformation, the curves overlap nearly perfectly within each set of sterols, with slopes of the curves for the sharp endotherms more flat for the $5 \mathrm{\alpha}-\mathrm{H}$ and 5,6 -double bond groups than the $5 \beta-\mathrm{H}$ group (3a-ol, 3a-ol, 3-K) (Fig $18 \mathrm{D}-\mathrm{F}$ ). For the broad component, the steroids are more perturbing for the $\mathrm{C} 5,6$-double bond and $5 \mathrm{\alpha}-\mathrm{H}$ groups than the corresponding sterols (3a-ol, $3 \alpha-o l)$ at all concentrations, as well as up to $30 \mathrm{~mol} \%$ for $5 \beta-\mathrm{H}, 3-\mathrm{K}$ when compared to $5 \beta-\mathrm{H}(3 \alpha-\mathrm{ol}, 3 \alpha-\mathrm{ol})$. Above $30 \mathrm{~mol} \%$, the net temperature shift for the broad component of $5 \beta-\mathrm{H}, 3 \beta-\mathrm{ol}$ drops significantly, making it the most perturbing sterol at high sterol concentrations (Fig $18 \mathrm{D}-\mathrm{I})$. This suggests that this sterol has a particularly unique packing arrangement that extremely disorders the bilayer structure.

\section{Discussion \\ Effects of sterols on DPPC model membranes: structure and thermodynamics}

The incorporation of a sterol molecule into the lipid bilayer disrupts the optimum packing between phospholipid molecules for a given phase structure. This produces a new packing geometry which will vary depending on the sterol/lipid mole ratio, the preference for self-association between like- and unlike-pairs arising from the directionality of associated local interactions, and the ability of water to enter into the bilayer interface. The relative rigidity of the sterol ring structure reduces the number of cis-trans isomerizations and kink formation in the neighbouring phospholipid acyl chains and has a differential disordering and ordering effect in the gel and $\mathrm{L}_{\mathrm{a}}$ phase bilayers, respectively (26). Thus, changes in sterol configuration and conformation, including small changes in the $\mathrm{OH}$ rotamer population, may alter the balance between attractive and repulsive interactions within the lipid bilayer matrix. Each sterol chemical configuration will have different contributions to bilayer thermodynamic and structural factors that are dependent on phase state $(30,31)$. Unfortunately, relatively little is known about the independent contributions of the sterol ring system and alkyl side chains. This project has attempted to do some analysis on the ring system.

\section{"Ideal” (C5 $\alpha-H)$ versus "Native" (C5,6 Double Bond) Ring Conformations}

The side-views of the 3D models of the C5,6 double bond and $5 \mathrm{a}-\mathrm{H}$ sterol ring systems shown in Figs 2 and 3 illustrate that there is a slight difference in the energy minimized structures between the two sterol rings systems, with the double bond in the C5,6 system introducing a slight torsion at the ring $\mathrm{A} / \mathrm{B}$ junction relative to the ideal, energyminimum all-trans ring system of the $\mathrm{C} 5 \mathrm{\alpha}-\mathrm{H}$ group with its 1,4 chair (cyclohexane) and envelope (cyclopentane) conformations. This structural change has mixed effects on the pretransition thermodynamic parameters. Chol and EChol are better at stabilizing the pretransition than their $5 \alpha-\mathrm{H}$ counterparts, whereas $5 \alpha-\mathrm{H}, \mathrm{K}$ is more effective than CholK. Similarly, the C5, 6 double bond group (3a-ol, 3a-ol, $3-\mathrm{K})$ is better at broadening the pretransition than the $\mathrm{C} 5 \mathrm{a}-\mathrm{H}$ group (3a-ol, 3a-ol, 3-K). In contrast, the C5a-H group $(3 \alpha-o l, 3 \alpha-o l, 3-K)$ is better at abolishing the pretransition with the exception of CholK, which is more effective than $5 \alpha-\mathrm{H}, 3-\mathrm{K}$. See Table 1 for a summary of these trends.

The trends in the thermodynamic parameters are more consistent for the sharp and broad components of the main transition, and show that the $5 \alpha-\mathrm{H}$ group (3a-ol, $3 \alpha-$ 
ol, 3-K) more effectively stabilizes the main transition sharp component at all concentrations and also stabilizes the broad component below $40 \mathrm{~mol} \%$. The $5 \mathrm{a}-\mathrm{H}$ group (3a-ol, $3 \alpha-o l, 3-K)$ also broadens and abolishes both main phase transition endotherms than the C5,6 group (3a-ol, 3a-ol, $3-\mathrm{K})$ at sterol concentrations below $\sim 25-30 \mathrm{~mol} \%$, above which the trend reverses, and $5 \alpha-\mathrm{H}, 3 \beta-\mathrm{ol}$, the closest structural analog to Chol, does not completely abolish the main transition at $50 \mathrm{~mol} \%$ sterol as does Chol. These findings suggest that the double bond in Chol increases sterol miscibility with the DPPC bilayer at high sterol concentrations, similar to those found in erythrocyte and ocular lens membranes which contain up to $50 \mathrm{~mol} \%$ and 70 mol\% Chol, respectively (32).

\section{"Distorted" (C5 $\beta-H)$ versus "Native" (C5,6 Double Bond) Ring Conformations}

The side views of the 3D-models of the $5 \mathrm{a}-\mathrm{H}$ sterols (Fig 4) illustrate that the $5 \alpha-\mathrm{H}$ conformation significantly deviates from the trans-planar steroid nucleus found in the native C5,6 double bond (3a-ol, 3a-ol, 3-K) and in the optimum $5 \alpha-\mathrm{H}$ groups (3a-ol, 3a-ol, 3-K). The trends in the thermodynamic parameters are more clear between the $\mathrm{C} 5,6$ double bond/5a-H groups (3a-ol, 3a-ol, 3-K) compared to the C5,6 double bond/5a-H groups ( $3 \alpha-$ ol, $3 \alpha-$ ol, 3-K). The C5,6 double bond group (3a-ol, 3a-ol, 3-K) is better able to stabilize the La, phase, while the C $5 \alpha-\mathrm{H}$ group $(3 \alpha-o l, 3 a-o l, 3-K)$ is better able to broaden and abolish the transition. An exception arises for $5 \alpha-\mathrm{H}, 3-\mathrm{K}$, which demonstrates the worst ability to abolish the transition of all the ketones. The significance of this observation will be discussed later, including the similarities in the cooperativity of the pretransition behaviour for the $5 \alpha-\mathrm{H}$ sterols and the steroids. See Table 1 for a summary of these trends.

For both the sharp and broad components of the main phase transition, we see that the $5 \alpha-\mathrm{H}$ sterols $(3 \alpha-$ ol, $3 \alpha-o l, 3-K)$ are the least able to stabilize the Pa, phase, although they are better able to broaden and abolish the phase transition of the sterol-poor domain. For the broad component at almost all sterol concentrations, the C5,6 double bond group ( $3 \alpha-\mathrm{ol}, 3 \alpha-\mathrm{ol}, 3-\mathrm{K})$ more effectively broadens and abolishes the phase transition of the sterolrich domain than the $\mathrm{C} 5 \alpha-\mathrm{H}$ group (3a-ol, 3a-ol, 3-K). This observed behaviour for the sterol-poor and sterolrich domains suggests that the kink introduced at the $A / B$ ring junction by the $5 \alpha-\mathrm{H}$ limits the number of favorable interactions between the sterols and lipids. The kink effectively increases the molecular cross-sectional area, which significantly destabilizes the sterol-poor bilayer regions. The overall increase in cross-sectional area can be compensated by a change in the molecular tilt of the DPPC molecule, but it would also reduce the number of molecular

\begin{tabular}{|c|c|c|c|c|c|c|c|c|c|c|}
\hline \multicolumn{11}{|c|}{ By C3 Functional Group } \\
\hline & & \multicolumn{3}{|c|}{$3 \beta-\mathrm{OH}$} & \multicolumn{3}{|c|}{$3 \alpha-\mathrm{OH}$} & \multicolumn{3}{|c|}{ 3-Ketone } \\
\hline & & Best & Middle & Least & Best & Middle & Least & Best & Middle & Least \\
\hline \multirow{3}{*}{$\begin{array}{l}\text { Pretransition } \\
\text { Ability to } \\
\text { Stabilize/ } \\
\text { Ability to } \\
\text { Broaden/ } \\
\text { Ability to } \\
\text { Abolish }\end{array}$} & $T_{p}$ & Chol & $5 \alpha-\mathrm{H}, 3 \beta-\mathrm{ol}$ & $5 \beta-\mathrm{H}, 3 \alpha-\mathrm{ol}$ & EChol & $5 \alpha-\mathrm{H}, 3 \beta-\mathrm{ol}$ & $5 \beta-\mathrm{H}, 3 \alpha-\mathrm{Ol}$ & $5 \alpha-\mathrm{H}, 3-\mathrm{K}$ & Cholk & $5 \beta-\mathrm{H}, 3-\mathrm{K}$ \\
\hline & $\Delta_{\mathrm{T} / \mathrm{p}}$ & $\begin{array}{l}5 \beta-\mathrm{H}, 3 \beta-\mathrm{ol} \\
(<4 \mathrm{~mol} \%) \\
\text { Chol } \\
(>4 \mathrm{~mol} \%)\end{array}$ & $5 \alpha-\mathrm{H}, 3 \beta-\mathrm{ol}$ & $\begin{array}{l}\text { Chol } \\
(<2 \text { mol\%) } \\
5 \beta-\mathrm{H}, 3 \beta-\mathrm{ol} \\
(>4 \mathrm{~mol} \%) \\
\end{array}$ & EChol & $5 \beta-H, 3 \alpha-$ ol & $5 \alpha-H, 3 \alpha-o l$ & $5 \beta-H, 3-K$ & Cholk & $5 \alpha-H, 3-K$ \\
\hline & $\Delta H_{p}$ & $5 \beta-\mathrm{H}, 3 \beta-\mathrm{Ol}$ & $5 \alpha-\mathrm{H}, 3 \beta-\mathrm{Ol}$ & Chol & $5 \alpha-H, 3 \alpha-\mathrm{Ol}$ & $5 \beta-H, 3 \alpha-\mathrm{Ol}$ & EChol & Cholk & $\begin{array}{l}5 \beta-\mathrm{H}, 3-\mathrm{K} \\
(>4 \mathrm{~mol} \%) \\
5 \alpha-\mathrm{H}, 3-\mathrm{K} \\
(<4 \text { mol\%) } \\
\end{array}$ & $\begin{array}{l}5 \alpha-\mathrm{H}, 3-\mathrm{K} \\
(<4 \mathrm{~mol} \%) \\
5 \beta-\mathrm{H}, 3-\mathrm{K} \\
(>4 \mathrm{~mol} \%)\end{array}$ \\
\hline \multirow{3}{*}{$\begin{array}{l}\text { Sharp } \\
\text { Component } \\
\text { Ability to } \\
\text { Stabilize/ } \\
\text { Ability to } \\
\text { Broaden/ } \\
\text { Ability to } \\
\text { Abolish }\end{array}$} & $T_{m}{ }^{\text {shp }}$ & $5 \alpha-\mathrm{H}, 3 \beta-\mathrm{ol}$ & Chol & $5 \beta-\mathrm{H}, 3 \beta-\mathrm{Ol}$ & $5 \alpha-H, 3 \alpha-\mathrm{ol}$ & EChol & $5 \beta-H, 3 \alpha-\mathrm{ol}$ & $5 \alpha-\mathrm{H}, 3-\mathrm{K}$ & Cholk & $5 \beta-\mathrm{H}, 3-\mathrm{K}$ \\
\hline & $\Delta \mathrm{T}_{1 / 2}^{\mathrm{shp}}$ & $5 \beta-\mathrm{H}, 3 \beta-\mathrm{ol}$ & $5 \alpha-\mathrm{H}, 3 \beta-\mathrm{Ol}$ & Chol & $5 \beta-\mathrm{H}, 3 \alpha-\mathrm{Ol}$ & EChol & $5 \alpha-\mathrm{H}, 3 \alpha-\mathrm{Ol}$ & $\begin{array}{l}5 \beta-\mathrm{H}, 3-\mathrm{K} \\
(<7 \mathrm{~mol} \%) \\
\text { CholK } \\
(>7 \mathrm{~mol} \%)\end{array}$ & $\begin{array}{l}\text { CholK } \\
(<7 \mathrm{~mol} \%) \\
5 \beta-\mathrm{H}, 3-\mathrm{K} \\
(>7 \mathrm{~mol} \%)\end{array}$ & $5 \alpha-\mathrm{H}, 3-\mathrm{K}$ \\
\hline & $\Delta \mathrm{H}_{\mathrm{m}}{ }^{\text {shp }}$ & $\begin{array}{l}5 \alpha-\mathrm{H}, 3 \beta-\mathrm{ol} \\
(<7 \mathrm{~mol} \%) \\
\text { Chol } \\
\text { (>7 mol\%) }\end{array}$ & $\begin{array}{l}\text { Chol } \\
\text { (<7 mol\%) } \\
5 \alpha-\mathrm{H}, 3 \beta-\mathrm{ol} \\
\text { (>7 mol\%) }\end{array}$ & $5 \beta-H, 3 \beta-\mathrm{Ol}$ & $5 \beta-\mathrm{H}, 3 \alpha-\mathrm{Ol}$ & EChol & $5 \alpha-H, 3 \alpha-\mathrm{ol}$ & $5 \beta-H, 3-K$ & $\begin{array}{l}5 \alpha-\mathrm{H}, 3-\mathrm{K} \\
(<15 \mathrm{~mol} \%) \\
\text { CholK } \\
\text { (>15 mol\%) }\end{array}$ & $\begin{array}{l}\text { CholK } \\
\text { (<15 mol\%) } \\
5 \alpha-\mathrm{H}, 3-\mathrm{K} \\
(>15 \mathrm{~mol} \%)\end{array}$ \\
\hline \multirow{3}{*}{$\begin{array}{l}\text { Broad } \\
\text { Component } \\
\text { Ability to } \\
\text { Stabilize/ } \\
\text { Ability to } \\
\text { Broaden/ } \\
\text { Ability to } \\
\text { Abolish }\end{array}$} & $T_{m}{ }^{\text {brd }}$ & $\begin{array}{l}5 \alpha-\mathrm{H}, 3 \beta-\mathrm{ol} \\
(<25 \mathrm{~mol} \%) \\
\text { Chol } \\
\text { (>25 mol\%) }\end{array}$ & $\begin{array}{l}\text { Chol } \\
\text { (<25 mol\%) } \\
5 \alpha-\mathrm{H}, 3 \beta-\mathrm{ol} \\
\text { (>25 mol\%) }\end{array}$ & $5 \beta-\mathrm{H}, 3 \beta-\mathrm{Ol}$ & $\begin{array}{l}5 \alpha-\mathrm{H}, 3 \alpha-\mathrm{ol} \\
(<25 \mathrm{~mol} \%) \\
\text { Chol } \\
\text { (>25 mol\%) }\end{array}$ & $\begin{array}{l}\text { Chol } \\
\text { (<25 mol\%) } \\
5 \alpha-H, 3 \alpha-o l \\
\text { (>25 mol\%) }\end{array}$ & $5 \beta-H, 3 \alpha-\mathrm{ol}$ & $5 \alpha-H, 3-K$ & $\begin{array}{l}\text { CholK } \\
\text { (<40 mol\%) } \\
5 \beta-\mathrm{H}, 3-\mathrm{K} \\
\text { (>40 mol\%) }\end{array}$ & $\begin{array}{l}5 \beta-\mathrm{H}, 3-\mathrm{K} \\
(<40 \mathrm{~mol} \%) \\
\text { CholK } \\
\text { (>40 mol\%) }\end{array}$ \\
\hline & $\Delta \mathrm{T}_{1 / 2}^{\text {brd }}$ & $\begin{array}{l}5 \beta-\mathrm{H}, 3 \beta-\mathrm{ol} \\
\text { (<25 mol\%) } \\
\text { Chol } \\
\text { (>25 mol\%) }\end{array}$ & $\begin{array}{l}\text { Chol } \\
\text { (<25 mol\%) } \\
5 \alpha-\mathrm{H}, 3 \beta-\mathrm{ol} \\
\text { (>25 mol\%) }\end{array}$ & $\begin{array}{l}5 \alpha-\mathrm{H}, 3 \beta-\mathrm{ol} \\
(<25 \mathrm{~mol} \%) \\
5 \beta-\mathrm{H}, 3 \beta-\mathrm{ol} \\
(>25 \mathrm{~mol} \%)\end{array}$ & EChol & $5 \beta-H, 3 \alpha-\mathrm{ol}$ & $5 \alpha-\mathrm{H}, 3 \alpha-\mathrm{Ol}$ & Cholk & $\begin{array}{l}5 \beta-\mathrm{H}, 3-\mathrm{K} \\
(<30 \mathrm{~mol} \%) \\
5 \alpha-\mathrm{H}, 3-\mathrm{K} \\
(>30 \mathrm{~mol} \%)\end{array}$ & $\begin{array}{l}5 \alpha-\mathrm{H}, 3-\mathrm{K} \\
(<30 \mathrm{~mol} \%) \\
5 \beta-\mathrm{H}, 3-\mathrm{K} \\
(>30 \mathrm{~mol} \%)\end{array}$ \\
\hline & $\Delta H_{m}{ }^{\text {brd }}$ & Chol & $5 \alpha-H, 3 \beta-$ ol & $5 \beta-H, 3 \beta-o l$ & $\begin{array}{l}5 \alpha-\mathrm{H}, 3 \alpha-\mathrm{ol} \\
(<30 \mathrm{~mol} \%) \\
\text { EChol } \\
(>30 \mathrm{~mol} \%)\end{array}$ & $\begin{array}{l}\text { EChol } \\
(<30 \mathrm{~mol} \%) \\
5 \alpha-\mathrm{H}, 3 \alpha-\mathrm{ol} \\
(>30 \mathrm{~mol} \%) \\
\end{array}$ & $5 \beta-\mathrm{H}, 3 \alpha-\mathrm{Ol}$ & $\begin{array}{l}5 \alpha-\mathrm{H}, 3-\mathrm{K} \\
(<40 \mathrm{~mol} \%) \\
\text { CholK } \\
(>40 \mathrm{~mol} \%) \\
\end{array}$ & $\begin{array}{l}\text { CholK } \\
\text { (<40 mol\%) } \\
5 \alpha-\mathrm{H}, 3-\mathrm{K} \\
\text { (>40 mol\%) }\end{array}$ & $5 \beta-\mathrm{H}, 3-\mathrm{K}$ \\
\hline $\begin{array}{l}\text { Total } \\
\text { Enthalpy } \\
\text { Ability to } \\
\text { Abolish }\end{array}$ & $\Delta \mathrm{H}_{\mathrm{m}}{ }^{\text {tot }}$ & $\begin{array}{l}5 \alpha-\mathrm{H}, 3 \beta-\mathrm{ol} \\
(<30 \mathrm{~mol} \%) \\
\text { Chol } \\
\text { (>30 mol\%) }\end{array}$ & $\begin{array}{l}\text { Chol } \\
\text { (<30 mol\%) } \\
5 \alpha-\mathrm{H}-3 \beta-\mathrm{ol} \\
\text { (>30 mol\%) }\end{array}$ & $5 \beta-H, 3 \beta-\mathrm{Ol}$ & EChol & $5 \alpha-H, 3 \alpha-\mathrm{ol}$ & $5 \beta-\mathrm{H}, 3 \alpha-\mathrm{Ol}$ & $\begin{array}{l}\text { CholK (<10 mol\%) } \\
5 \alpha-\mathrm{H}, 3-\mathrm{K} \text { (>10 } \\
\mathrm{mol} \%,<40 \mathrm{~mol} \%) \\
\text { CholK } \\
\text { (>40 mol\%) }\end{array}$ & $\begin{array}{l}\text { CholK (>15 } \\
\text { mol\%, } \\
<40 \text { mol\%) } \\
5 \alpha-\mathrm{H}, 3-\mathrm{K} \\
(>40 \mathrm{~mol} \%)\end{array}$ & $\begin{array}{l}5 \beta-\mathrm{H}, 3-\mathrm{K} \\
(>15 \mathrm{~mol} \%)\end{array}$ \\
\hline
\end{tabular}

Table 1. Observation summaries for the pretransition (Pretransition row) and main phase transition (Sharp Component, Broad Component, and Total Enthalpy rows) arranged by C3 functional group $(3 \beta-0$, , $3 \alpha-0 l, 3-K) . T\left({ }^{\circ} \mathrm{C}\right)$ ranks the ability of the sterols to stabilize either the L $\beta^{\prime}$ (pretransition) or P $\beta^{\prime}$ (main phase transition) phases, $\Delta T 1 / 2\left({ }^{\circ} \mathrm{C}\right)$ ranks the ability of the sterols to broaden the respective transition, and $\Delta H(\mathrm{kcal} / \mathrm{mol})$ ranks the ability of the sterols to abolish the respective transition. 
contacts within the hydrocarbon chain region. As a consequence, the overall number of favorable interactions with the lipid bilayer would decrease more rapidly for DPPC mixtures containing a kinked sterol than for sterols with smaller cross-sectional areas like the $\mathrm{C} 5 \mathrm{\alpha}-\mathrm{H}$ group ( $3 \alpha-\mathrm{ol}$, $3 \alpha-\mathrm{ol}, 3-\mathrm{K}$ ). This may explain why the $\mathrm{C} 5 \alpha-\mathrm{H}$ group ( $3 \alpha-\mathrm{ol}$, $3 \alpha-0,3-K)$ much more rapidly reaches its miscibility limit in the sterol-rich DPPC domain.

\section{$3 \alpha$-ols versus $3 \beta$-ols}

A comparison of the DSC measurements obtained for the pretransition of DPPC mixtures containing low concentrations of either $3 \alpha$-ols or $3 \alpha$-ols whose hydroxyl group orientations are equatorial and axial, respectively, found that the $3 \alpha$-ols more effectively stabilize the $L \alpha$ ' phase and broaden the pretransition more effectively than the corresponding 3a-ols. See Table 2 for a summary of these trends. The same relationship exists between $\mathrm{OH}-$ group orientation and the ability of the sterol to abolish the pretransition, with the exception of the $5 \alpha-\mathrm{H}$ group, where the $3 \alpha-o l$ is more effective than the $3 \alpha-o l$. In the case of the sharp component of the main transition, $3 \alpha$-ols more effectively stabilize the Pa' phase and broaden the sterolpoor domain phase transition than the $3 \alpha$-ols. However, the sharp component persists up to 40 and $50 \mathrm{~mol} \%$ in both EChol and $5 \mathrm{a}-\mathrm{H}, 3 \mathrm{a}-\mathrm{ol}$, respectively, and suggests that there is a change in the balance of sterol- poor and sterol-rich stoichiometries in favor of the former. Within the main phase transition broad component of the $3 \alpha / \alpha-$ ols, Chol (3a-ol) better stabilizes the gel phase than EChol (3a-ol), $5 \alpha-H, 3 \alpha-o l ~(3 \alpha-o l)$ better stabilizes the gel phase than $5 \alpha-\mathrm{H}, 3 \alpha-\mathrm{ol}(3 \alpha-\mathrm{ol})$ at $>40 \mathrm{~mol} \%$ sterol concentrations and $5 \alpha-H, 3 \alpha-o l(3 \alpha-o l)$ better stabilizes the gel phase than
$5 \alpha-\mathrm{H}, 3 \alpha-\mathrm{ol}(3 \alpha-\mathrm{ol})$ at all sterol concentrations. However, in all cases, the $3 a$-ols always more effectively broaden and abolish the broad endotherms than the $3 \alpha$-ols and more so at high sterol concentrations. Previous work with EChol suggested that the sterol sits lower in the bilayer interface, which would result in decreased hydrogen-bonding interactions at the lipid-water interface (24). It appears that we are looking at two perturbing mechanisms here, one related to cross-sectional area and the other to the sterol depth in the bilayer. DSC alone, however, is unable to distinguish the extent to which either of these mechanisms perturbs bilayer structure.

\section{Steroids versus Sterols}

There are few consistent relationships between the steroids and the sterols other than the steroids $(5 \alpha-\mathrm{H}$, $5 \alpha-\mathrm{H}, \mathrm{C} 5,6$ double bond) show significant inabilities to stabilize, broaden and abolish both the pretransition and main-chain melting transitions relative to the sterols. However, especially notable are the significant differences in $\Delta T_{p 1 / 2}$ in the steroids $(5 \alpha-H, 5 \alpha-H, C 5,6$ double bond) (and also $5 \alpha-\mathrm{H}, 3 \alpha-\mathrm{ol}$ and $5 \alpha-\mathrm{H}, 3 \alpha-\mathrm{ol}$ ) with increasing sterol concentration, suggesting the presence of multiple endothermic peaks in the pretransition of these sterol/DPPC mixtures. As suggested earlier, the major contributor to this behaviour for the $5 \alpha-\mathrm{H}$ group may arise from the kink in the A-B ring junction, which increases the cross-sectional area of the steroid nucleus and preventing molecular packing and favourable interactions with neighbouring DPPC molecules. This could increase the number of chemical environments seen by the sterol molecule. A similar behaviour may occur upon oxidizing the $\mathrm{C} 3-\mathrm{OH}$ functional group to a ketone, where the polarizability of

\begin{tabular}{|c|c|c|c|c|c|c|c|c|c|c|}
\hline \multicolumn{11}{|c|}{ By C5 Conformation } \\
\hline & & \multicolumn{3}{|c|}{ 5,6-double bond } & \multicolumn{3}{|c|}{$\underline{5 \alpha-\mathrm{H}}$} & \multicolumn{3}{|c|}{$5 \beta-H$} \\
\hline & & Best & Middle & Least & Best & Middle & Least & Best & Middle & Least \\
\hline \multirow{3}{*}{$\begin{array}{l}\text { Pretransition } \\
\text { Ability to } \\
\text { Stabilize/ } \\
\text { Ability to } \\
\text { Broaden/ } \\
\text { Ability to } \\
\text { Abolish }\end{array}$} & $T_{p}$ & EChol & Chol & Cholk & $5 \alpha-H, 3-K$ & $5 \alpha-H, 3 \alpha-$ ol & $5 \alpha-\mathrm{H}, 3 \beta-\mathrm{ol}$ & $5 \beta-\mathrm{H}, 3 \alpha-\mathrm{ol}$ & $5 \beta-H, 3 \beta-$ ol & $5 \beta-H, 3-K$ \\
\hline & $\Delta_{\mathrm{T} / 2 \mathrm{p}}$ & EChol & Chol & Cholk & $5 \alpha-\mathrm{H}, 3 \beta-\mathrm{ol}$ & $5 \alpha-H, 3 \alpha-o l$ & $5 \alpha-H, 3-K$ & $5 \beta-H, 3 \alpha-o l$ & $5 \beta-H, 3-K$ & $5 \beta-\mathrm{H}, 3 \beta-\mathrm{ol}$ \\
\hline & $\Delta H_{p}$ & Cholk & EChol & Chol & $5 \alpha-\mathrm{H}, 3 \alpha-\mathrm{ol}$ & $\begin{array}{l}5 \alpha-\mathrm{H}, 3 \beta-\mathrm{ol} \\
(<4 \mathrm{~mol} \%) \\
5 \alpha-\mathrm{H}, 3-\mathrm{K} \\
(>4 \mathrm{~mol} \%)\end{array}$ & $\begin{array}{l}5 \alpha-\mathrm{H}, 3-\mathrm{K} \\
(<4 \text { mol\%) } \\
5 \alpha-\mathrm{H}, 3 \beta-\mathrm{ol} \\
\text { (>4 mol\%) }\end{array}$ & $5 \beta-H, 3 \beta-o l$ & $5 \beta-H, 3-K$ & $5 \beta-H, 3 \alpha-\mathrm{ol}$ \\
\hline \multirow{3}{*}{$\begin{array}{l}\text { Sharp } \\
\text { Component } \\
\text { Ability to } \\
\text { Stabilize/ } \\
\text { Ability to } \\
\text { Broaden/ } \\
\text { Ability to } \\
\text { Abolish }\end{array}$} & $T_{m}^{\text {shp }}$ & Chol & EChol & Cholk & $5 \alpha-\mathrm{H}, 3 \beta-\mathrm{ol}$ & $5 \alpha-H, 3 \alpha-$ ol & $5 \alpha-H, 3-K$ & $5 \beta-H, 3 \beta-o l$ & $5 \beta-H, 3-K$ & $5 \beta-H, 3 \alpha-\mathrm{ol}$ \\
\hline & $\Delta \mathrm{T}_{1 / 2}^{\text {shp }}$ & Cholk & $\begin{array}{l}\text { EChol } \\
\text { (<15 mol\%) } \\
\text { Chol } \\
\text { (>15 mol\%) }\end{array}$ & $\begin{array}{l}\text { Chol } \\
\text { (<15 mol\%) } \\
\text { Echol } \\
\text { (>15 mol\%) }\end{array}$ & $5 \alpha-\mathrm{H}, 3 \beta-\mathrm{ol}$ & $5 \alpha-H, 3-K$ & $5 \alpha-\mathrm{H}, 3 \alpha-\mathrm{ol}$ & $\begin{array}{l}5 \beta-\mathrm{H}, 3-\mathrm{K} \\
(<10 \mathrm{~mol} \%) \\
5 \beta-\mathrm{H}, 3 \beta-\mathrm{ol} \\
(>10 \mathrm{~mol} \%)\end{array}$ & $\begin{array}{l}5 \beta-\mathrm{H}, 3 \beta-\mathrm{ol} \\
(<10 \mathrm{~mol} \%) \\
5 \beta-\mathrm{H}, 3-\mathrm{K} \\
(>10 \mathrm{~mol} \%)\end{array}$ & $5 \beta-H, 3 \alpha-\mathrm{ol}$ \\
\hline & $\underset{\text { shp }}{\Delta \mathbf{H}_{\mathrm{m}}}$ & $\begin{array}{l}\text { EChol } \\
\text { (<20 mol\%) }\end{array}$ & Chol & Cholk & $5 \alpha-\mathrm{H}, 3 \beta-\mathrm{ol}$ & $5 \alpha-H, 3-K$ & $5 \alpha-H, 3 \alpha-$ ol & $\begin{array}{l}5 \beta-H, 3-K= \\
5 \beta-H, 3 \alpha-\text { ol }\end{array}$ & $\begin{array}{l}5 \beta-H, 3-K= \\
5 \beta-H, 3 \alpha-\text { ol }\end{array}$ & $5 \beta-H, 3 \beta-\mathrm{ol}$ \\
\hline \multirow{3}{*}{$\begin{array}{l}\text { Broad } \\
\text { Component } \\
\text { Ability to } \\
\text { Stabilize/ } \\
\text { Ability to } \\
\text { Broaden/ } \\
\text { Ability to } \\
\text { Abolish }\end{array}$} & $T_{m}{ }^{b r d}$ & Chol & EChol & Cholk & $\begin{array}{l}5 \alpha-\mathrm{H}, 3 \beta-\mathrm{ol} \\
(<40 \mathrm{~mol} \%) \\
5 \alpha-\mathrm{H}, 3 \alpha-\mathrm{ol} \\
(>40 \mathrm{~mol} \%)\end{array}$ & $\begin{array}{l}5 \alpha-H, 3 \alpha-\mathrm{ol} \\
(<40 \mathrm{~mol} \%) \\
5 \alpha-\mathrm{H}, 3 \beta-\mathrm{ol} \\
\text { (>40 mol\%) }\end{array}$ & $5 \alpha-H, 3-K$ & $5 \beta-H, 3 \alpha-$ ol & $\begin{array}{l}5 \beta-\mathrm{H}, 3 \beta-\mathrm{ol} \\
(<30 \mathrm{~mol} \%) \\
5 \beta-\mathrm{H}, 3-\mathrm{K} \\
(>30 \mathrm{~mol} \%)\end{array}$ & $\begin{array}{l}5 \beta-\mathrm{H}, 3 \beta-\mathrm{ol} \\
\text { (<30 mol\%) } \\
5 \beta-\mathrm{H}, 3-\mathrm{K} \\
(>30 \mathrm{~mol} \%)\end{array}$ \\
\hline & $\Delta T_{1 / 2}^{\text {brd }}$ & Chol & EChol & Cholk & $5 \alpha-\mathrm{H}, 3 \beta-\mathrm{ol}$ & $5 \alpha-H, 3-K$ & $5 \alpha-\mathrm{H}, 3 \alpha-\mathrm{ol}$ & $5 \beta-H, 3 \beta-o l$ & $5 \beta-H, 3-K$ & $5 \beta-H, 3 \alpha-\mathrm{Ol}$ \\
\hline & $\underset{\text { brd }}{\Delta H_{m}}$ & $\begin{array}{l}\text { EChol } \\
\text { (<40 mol\%) } \\
\text { Chol } \\
\text { (>40 mol\%) }\end{array}$ & $\begin{array}{l}\text { Chol } \\
\text { (<40 mol\%) } \\
\text { EChol } \\
\text { (>40 mol\%) }\end{array}$ & Cholk & $\begin{array}{c}5 \alpha-\mathrm{H}, 3 \alpha-\mathrm{ol} \\
<40 \mathrm{~mol} \%) \\
5 \alpha-\mathrm{H}, 3 \beta-\mathrm{ol} \\
(>40 \mathrm{~mol} \%)\end{array}$ & $\begin{array}{l}5 \alpha-\mathrm{H}, 3 \beta-\mathrm{ol} \\
(<40 \mathrm{~mol} \%) \\
5 \alpha-\mathrm{H}, 3 \alpha-\mathrm{ol} \\
(>40 \mathrm{~mol} \%)\end{array}$ & $5 \alpha-H, 3-K$ & $5 \beta-H, 3 \beta-o l$ & $5 \beta-H, 3-K$ & $5 \beta-H, 3 \alpha-\mathrm{ol}$ \\
\hline
\end{tabular}

Table 2. Observation summaries for the pretransition (Pretransition row) and main phase transition (Sharp Component, Broad Component, and Total Enthalpy rows) arranged by $\mathrm{C} 5$ conformation $(5,6$ double-bond, $5 \alpha-\mathrm{H}, 5 \beta-\mathrm{H}) . \mathrm{T}\left({ }^{\circ} \mathrm{C}\right)$ ranks the ability of the sterols to stabilize either the $\mathrm{L} \beta^{\prime}$ (pretransition) or P $\beta^{\prime}$ (main phase transition) phases, $\Delta \mathrm{T} 1 / 2\left({ }^{\circ} \mathrm{C}\right)$ ranks the ability of the sterols to broaden the respective transition, and $\Delta \mathrm{H}(\mathrm{kcal} / \mathrm{mol})$ ranks the ability of the sterols to abolish the respective transition. 
the nuclear polar group is reduced and the ability of the steroid to act as a hydrogen bond donor to the carbonyl of the acyl chains in the DPPC bilayers is eliminated, meaning that hydrogen bonding is only possible with water. One possible explanation for this behaviour is that the rippled gel phase exists in two isomorphs differing in periodicity and that the steroids (5a-H, 5a-H, C5,6 double bond) and the $5 \alpha-\mathrm{H}$ sterols ( $3 \alpha-\mathrm{ol}, 3 \alpha-\mathrm{ol})$ shifts the balance between these two related phase structures (33).

Reduced polarizability and an inability to donate hydrogen bonds would suggest that the steroids sit much lower in the bilayer. This idea has been confirmed by molecular model simulations $(34,35)$, and these same simulations illustrate that substituting the hydroxyl group with a ketone promotes the translocation or flip-flopping of steroids between the leaflets. Further, 3-ketone functional groups are usually found in nature in steroid hormones like testosterone and estrogen that usually must diffuse or be transported through cellular bilayers to reach their target, like the cellular nucleus where they activate transcription factors. This suggests then that the ketone group prevents steroids from having a prolonged residence in either membrane leaflet. Hence, the combination of the $5 \alpha-\mathrm{H}$ conformation and a ketone functional group would create a steroid with extreme miscibility problems (discussed in next section).

The deeper penetration of sterols has been postulated to increase the ordering and condensation of PC bilayers (36, 37). This has been supported by Chol/EChol studies using both FTIR (Chol/EChol) and molecular simulations $(24,38)$, which implies that further information as to where these sterols are sitting in the bilayer may come to light though Fourier transform infrared spectroscopy studies. While DSC can provide valuable thermodynamic data about the phase state and organization of lipid assemblies and how these structure and physical properties are modulated by membrane constituents, it is not a structural technique. Hence, DSC is most valuable at interpretation sterol/lipid interactions when it is combined with a direct structural technique such as FTIR, NMR, or X-ray diffraction (39).

\section{Sterol Immiscibility and Optimal Lipid-Sterol Stoichiometries}

At the boundary between sterol-poor and sterol-rich domains, the further addition of sterol into the DPPC bilayer does not result in a homogenous sterol distribution in the bilayer but the creation of different lipid/sterol microdomains (40). These domains exist in both gel and liquid-crystalline phase regions and may differ in both their physical properties and composition up to a critical miscibility point. Hence, similar slopes below $\Delta \mathrm{H}_{\mathrm{m}}{ }^{\text {brd-max }}$ within a subset (Figs 10-15 F) suggest similar sterol/DPPC stoichiometries or domain structures for the parent chemical configuration (for example, 5a-H, 5a-H, C5,6 double bond), whereas significant differences in slope probably reflect a change in the balance between the various lipid/sterol domains or a change in domain structure.

$\Delta \mathrm{H}_{\mathrm{m}}$ brd-max represents a critical miscibility point where at least two domains containing different sterol-lipid populations coalesce. At higher sterol concentrations, the sterol/lipid dispersion is uniform and increasing amounts of sterol partition uniformly into the bilayer, effectively increasing the sterol/lipid ratio. However, full miscibility can only occur if the balance between like/like and like/unlike pairs favours the latter. When the like/unlike interactions are strong and the molecular packing is ideal, $\Delta \mathrm{H}_{\mathrm{m}}$ brd $=0$, as is seen for Chol. This means that if $\Delta \mathrm{H}_{\mathrm{m}}$ brd $\neq 0$ for specific sterol/DPPC mixtures, then either the balance between like/like and like/unlike pairs is not ideal, or there are fewer or weaker favorable interactions between like/unlike pairs. Therefore, full miscibility is not achieved and may not be possible. Also, differences in the magnitude of $\Delta \mathrm{H}_{\mathrm{m}}$ brd-max among different sterol chemical structures probably arise from the different ability of each sterol to disrupt the lipid/ sterol interactions of the dominant domain. A possible reason for this may include the formation of a highlyordered molecular packing with very strong like/unlike interactions. Thus, by virtue of differences in the strength and number of like/unlike interactions, each sterol structure will determine the number of different lipid domains, their composition, and, potentially, their physical structure. The formation of each domain structure will occur at a critical point away from or close to the phase transition of the mixture (41-43). The stability of each domain is determined by the ease with which additional sterol molecules can be incorporated into that specific domain structure. "Stable" domains have a greater sterol insertion energy which will vary with sterol structure and will also be concentration dependent. This would explain the variation in $\Delta \mathrm{H}_{\mathrm{m}}$ brd-max in both the concentration and enthalpy context.

An example of immiscibility in these DPPC mixtures where the balance between like/like and unlike/like interactions is not altered is the $5 \alpha-\mathrm{H}$ sterol conformation where the kink at the ring $A / B$ junctions increases the overall steric bulk of the molecule. The effect of the change in the ring $A$ orientation cannot explain the significantly greater $\Delta \mathrm{H}_{\mathrm{m}}^{\text {brd-max }}$ and the inability of $5 \alpha-\mathrm{H}, 3 \alpha-\mathrm{ol}$ to abolish the phase transition relative to that of $5 \alpha-\mathrm{H}, 3 \alpha-$ ol. Therefore, the observations for the $5 \alpha-\mathrm{H}$ sterols $(3 \alpha-$ ol, $3 \alpha-o l)$ suggest that if the earlier interpretation of the $\Delta \mathrm{H}_{\mathrm{m}}{ }^{\text {brd-max }}$ is valid, the combination of the $3 \alpha-\mathrm{OH}$ and $5 \beta$ $\mathrm{H}, 10 \mathrm{~b}-\mathrm{CH}_{3}$ (cis) configurations produces a sterol molecule that has fewer favorable interactions in the interfacial and hydrocarbon chair regions with neighboring DPPC molecules and, therefore, cannot form stable domains in the DPPC bilayer. This sterol would be unable to maintain a high sterol/PC concentration in the bilayer and would not be fully miscible with DPPC. In the corresponding $5 \beta-\mathrm{H}, 3 \beta-$ ol, partial miscibility is restored by stronger intermolecular interactions with DPPC in the bilayer interface with the change in C3-hydroxyl orientation.

The inability of all the sterols and steroids studied here other than Chol to abolish the main phase transition at high sterol concentrations has been noted with other sterols (23-25) and comes as no surprise if Chol is assumed to have to have the ideal structure for packing and optimal miscibility in DPPC bilayers. The location of the immiscible sterol pool is not clear when DSC in unable to detect those separate phases like those found in the $5 \beta-\mathrm{H}, 3-\mathrm{K} /$ DPPC mixtures, where phase transitions arising from 
phase separated crystalline sterols is observed at higher steroid concentrations (Fig 16). The significant increase in the area of the crystallite endotherm above the phase transition is accompanied by the sudden increase in $T_{m}$ brd and decrease in $\Delta \mathrm{T}_{1 / 2}$ brd at $50 \mathrm{~mol} \%$ concentration for $5 \beta-H, 3-K$ (Fig 12 D,E). Nucleation of crystalline $5 \alpha-H, 3-K$ occurs in the bilayer and then the sterol partitions out into the bulk phase changing the thermodynamic parameters of the $50 \%$ mol $5 \alpha-H, 3-K / D P P C$ mixture to one more closely resembling a sample containing $25-30$ mol\% steroid. To a lesser degree, these same changes in $T_{m}$ brd and $\Delta T_{1 / 2}$ brd are seen for $5 \alpha-\mathrm{H}, 3-\mathrm{K}$, although no crystallites are visible in thermograms obtained from mixtures containing higher sterol concentrations. Their absence may be due to a lack of instrument sensitivity and the poorly-energetic nature of these weak transitions. This is plausible given that the total enthalpy curves for both the $5 \mathrm{a}-\mathrm{H}$ and $5 \mathrm{a}-\mathrm{H}$ steroids level off rapidly above $30 \mathrm{~mol} \%$ concentration. Interestingly, the total enthalpy curve does not level off as readily for CholK and is the most able of the steroids to abolish the DPPC main transition. CholK also shows a significant ability to perturb the DPPC bilayers at these sterol concentrations, lending additional support to the conclusion made earlier that the C5,6 double bond provides additional stabilizing interactions with DPPC in the bilayer at physiologicallyrelevant sterol concentrations.

\section{Concluding Remarks}

The Venn diagram presented in Fig 19 summarizes the ability of the various sterols and steroids studied to stabilize the Pa, phase and broaden and abolish the DPPC $\mathrm{Pa}, / \mathrm{La}$ phase transition. Chol is best able to stabilize the $\mathrm{Pa}$, phase and broaden and abolish the DPPC main phase transition. Moving outwards, the other C5,6 double bond (EChol, CholK) and C5a-H group members (3a-ol, 3a-ol, $3-K)$ show abilities to meet at least two of these criteria. The $5 \alpha-\mathrm{H}$ group (3a-ol, 3a-ol, 3-K) shows significantly diminished capabilities, with $5 \mathrm{a}-\mathrm{H}, 3-\mathrm{K}$ being distinct from other members of the global set. In a general sense, we see that an all-trans ring system is required to stabilize the $\mathrm{Pa}$, phase, a $3 \alpha-\mathrm{OH}$ functional group to broaden the transition, and the presence of the C5,6-double bond to abolish the transition. Hence, changes in the chemical structure of the sterol nucleus influence the bilayer thermodynamic properties, such as the gel/ $L_{a}$ phase transition temperature and cooperativity, the ability to abolish the chain-melting phase transition and the miscibility of the sterol in the lipid bilayer. The changes in sterol chemical structure are also known to affect the molecular tilt (44), the headgroup/ hydrocarbon chain packing geometry, hydration, curvature and, consequently, the membrane nonlamellar phase preference (45-47). Our observations lend support to the Bloch hypothesis, which suggested that there was a progressive change in the ability of the intermediates in Chol biosynthesis to pack into a phospholipid bilayer and that lanosterol (the earliest intermediate) is the poorest fit and Chol (the end product) is the best. This proposes that this energy-expensive conversion process, which produces a sterol with optimum molecular packing abilities in the phospholipid bilayer, has considerable evolutionary

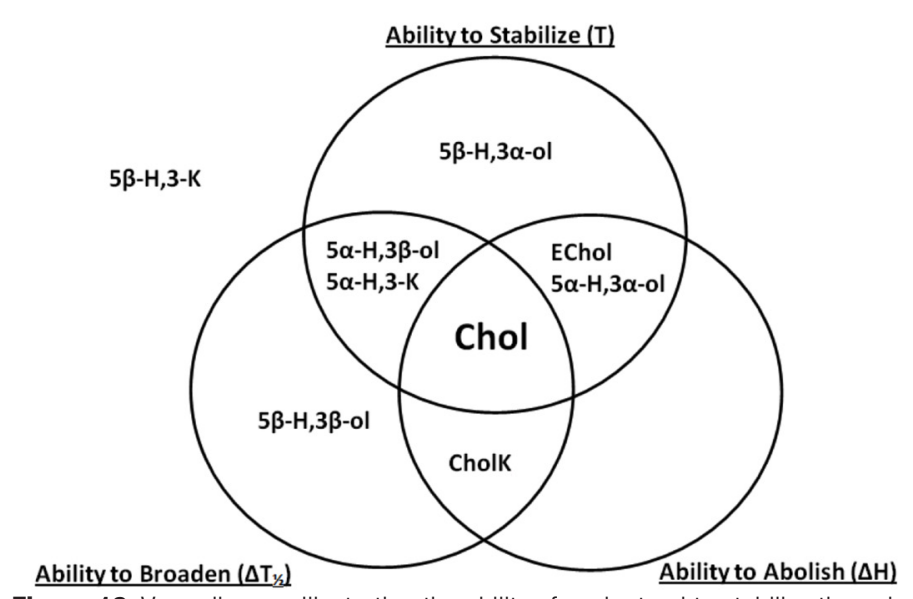

Figure 19. Venn diagram illustrating the ability of each sterol to stabilize the gel phase $(T)$, and broaden $(\triangle T 1 / 2)$ and abolish the main phase transition $(\Delta H)$ at high (biologically relevant) sterol concentrations. Cholesterol meets all three parameters while $5 \beta-H, 3-K$ fails to reasonably satisfy any of the parameters relative to the other sterols studied. Overall, sterols with a $\mathrm{C} 5,6$ double bond and $\mathrm{C} 5 \mathrm{\alpha}-\mathrm{H}$ conformation are best able to stabilize the $\mathrm{L}^{\prime}$ ' phase. The $3 \beta-\mathrm{OH}$ sterols are the best at broadening the main phase transition, while the sterols with the $\mathbf{C 5 , 6}$ double bond conformation are best able to abolish the main phase transition.

significance (48).

Given the differences in the thermodynamic parameters obtained from DPPC mixtures containing different sterol ring configurations and their associated changes in bilayer stability and miscibility, it becomes clear that changing the sterol chemical configuration has a significant effect on the bilayer physical properties. Thus, any sterol molecule whose ring structure deviates from that of Chol is unlikely to be fully miscible in the mammalian cell membrane, and an in vivo change in ring structure brought about by, for example, oxidation of $3 \mathrm{a}-\mathrm{OH}$ by Chol oxidase followed by the removal of the SpM headgroup would destabilize the physical structure of the lipid rafts. Such an event, which has been reported in many human diseases (see the Introduction), would result in a signaling cascade leading to cell death and disease manifestation. Hence, understanding the variation in the strength, number, and type of interactions between the sterol ring system and neigbouring phospholipid molecules is an important part of understanding the disease process and prospective membrane-associated therapies.

\section{Acknowledgements}

This work was funded by operating and equipment grants from the Alberta Heritage Foundation for Medical Research and by the Canadian Institutes of Health Research. Special thanks to Dr. Ruthven N.A.H. Lewis for his ongoing assistance.

\section{References}

1. Hartmann, T., Kuchenbecker, J., Grimm, M.O.W. (2007) J. Neurochem. 103: 159-170.

2. D’Errico, G., Vitiello, G., Ortona, O., Tedeschi, A., Ramunno, A., D’Ursi, A.M. (2008). Biochim. Biophys. Acta. 1778: 2710-2716.

3. Sacchetti, P., Sousa, K.M., Hall, A.C., Liste, I., Steffensen, K.R., Theofilopoulos, S., Parish, C.L., Hazenberg, C., Richter, L.A., Hovatta, O., Gustafsson, J.-A., Arenas, E. (2009) Cell Stem Cell 5: 409-419.

4. Chazal, N., Gerlier, D. (2003) Microbiol. Mol. Biol. Rev. 67: 226-37.

5. Ablan, S., Rawat, S.S., Viard, M., Wang, J.M., Puri, A., Blumenthal, R. 
(2006) Virol. J. 3: 104. PMID: 17187670.

6. Waheed, A.A., Freed, E.O., (2009) Virus Res. 143: 162-176.

7. Wadia, J.S., Schaller, M., Williamson, R.A., Dowdy, S.F. (2008) PLoS ONE 3: e3314. doi:10.1371/journal.pone.0003314.

8. Zhong, J., Yang, C., Zheng, W., Huang, L., Hong, Y., Wang, L., Sha, Y. (2009) Biophys. J. 96: 4610-21. Erratum in: Biophys J. 97: 397.

9. van der Meer-Janssen, Y.P.M., van Galen, J., Batenburg, J.J., Helms, J.B. (2010) Prog. Lipid Res. 49: 1-26.

10. Panda, S., Jafri, M., Kar, A., Meheta, B.K. (2009) Fitoterapia 80: 123126.

11. Woyengo, T.A., Ramprasath, V.R., Jones, P.J.H. (2009) Eur. J. Clin. Nutr. 63: 813-820.

12. Carpinteiro, A., Dumitru, C., Schenck, M., Gulbins, E. (2008) Cancer Lett. 264: 1-10.

13. Barenholz, Y. (2004) Subcell. Biochem. 37: 167-215.

14. Cremesti, A.E., Goni, F.M., Kolesnick, R. (2002) FEBS Lett. 531: 4753.

15. Biasi, F., Mascia, C., Astegiano, M., Chiarpotto, E., Nano, M., Vizio, B., Leonarduzzi, G., Poli, G. (2009) Free Rad. Biol. Med. 47: 17311741.

16. Ramstedt, B., Slotte, J.P. (2006) Biochim. Biophys. Acta 1758: 194556.

17. Goñi, F.M., Alonso, A. (2009) Biochim. Biophys. Acta. 1788: 169-77.

18. Maggio, B., Borioli, G.A., Del Boca, M., De Tullio, L., Fanani, M.L., Oliveira, R.G., Rosetti, C.M., Wilke, N. (2008) Cell Biochem. Biophys. 50: 79-109.

19. Mannock, D.A., McIntosh, T.J., Jiang, X., Covey, D.F., McElhaney, R.N. (2003) Biophys. J. 84: 1038-1046.

20. McMullen, T.P.W., Lewis, R.N.A.H., McElhaney. R.N. (1993) Biochemistry. 32: 516-522.

21. McMullen, T.P.W., Lewis, R.N.A.H., McElhaney. R.N. (1994) Biophys. J. 66: 741-752.

22. McMullen, T.P., Lewis, R.N.A.H., McElhaney, R.N. (2009) Biochim. Biophys. Acta 1788: 345-57.

23. Mannock, D.A., Lewis, R.N.A.H., McElhaney, R.N. (2006) Biophys. J. 91: 3327-3340.

24. Mannock, D.A., Lee, M.Y., Lewis, R.N.A.H., McElhaney, R.N. (2008) Biochim. Biophys. Acta. 1778: 2191-202.

25. Mannock, D.A., Lewis, R.N.A.H., McElhaney, R.N. (2010) Biochim. Biophys. Acta. 1798: 376-388.

26. Mannock, D.A., Lewis, R.N.A.H., McMullen, T.P.W., McElhaney, R.N. (2010) CPL. doi:10.1016/j.chemphyslip.2010.03.011.

27. McNamara, D.J., Pria, A, Miettinen, T.A. (1981) J. Lipid Res. 22: 474-
484.

28. Javiltt, N.B. (2002) BBRC. 292: 1147-1153.

29. Lewis, R.N.A.H., Mannock, D.A., McElhaney, R.N. (2007) Differential scanning calorimetry on the study of lipid phase transitions in mode and biological membranes: practical considerations, in: A. Dopico (Ed.), Methods in Membrane Lipids, Humana Press, Totown, New Jersey, pp. 171-195.

30. Quindnero, D., Frontera, A., Escudero, D., Ballester, P., Costa, A., Deya, P.M. (2008) Theor. Chem. Account. 120: 385-293.

31. Estarellas, C., Frontera, A., Qunidenero, D., Alkorla, I, Deya, P.M. (2009) J. Phys. Chem. A. 113: 3266-3273.

32. Rog, T., Pasenkiewicz-Gierula, M, Vallulainen, I., Karttunen, M. (2009) Biochim. Biophys. Acta. 1788: 97-121.

33. Katsaras, J., Kucerka, N., Nieh, M. (2008) Biointerphases. 3: F55-F63.

34. Rog, T., Stimson, L.M., Paskenkewicz-Gierula, M., Vallulainen, I., and Karttunen, M. (2008) J. Phys. Chem. B. 112, 1946-1952.

35. Berring, E.E., Borrenphol, K., Fliesler, S.J., Serfis, A.R.. (2005) CPL. 136: 1-12.

36. Rog, T., and Paskenkewicz-Gierula, M. (2006) Biophys. J. 91, 37563767.

37. Rog, T., and Paskenkewicz-Gierula, M. B(2006) iochemie. 88, 449460.

38. Rog, T., and Pasenkiewicz-Gierula, M. (2003) Biophys. J. 84, 18181826.

39. Lewis, R.N.A.H, Mannock, D.A., McElhaney, R.N. (2008) Differential Scanning Calorimetry to Study Lipids and Lipid Membranes, in Wiley Encyclopedia of Chemical Biology. John Wiley \& Sons, Inc. doi: 10.1002/9780470048672.wecb049.

41. Veatch, S.L., Sunbias, O., Keller, S.L., Ganrisch, K. (2007). PNAS. 104: 17650-17655.

42. Veatch, S.L. (2007). SCDB. 16: 573-582.

43. Veatch, S.L., Keller, S.L., Biochim. Biophys. Acta. (2005). 1746: 172285.

44. Cournia, Z, Ullmann, G.M., Smith, J.C. (2007) J Phys. Chem. B. 111: 1786-1801.

45. Wang X., Quinn, P.J. (2002). Biochim. Biophys. Acta. 1564: 66-72.

46. Bacia, K., Schwille, P., Kurzchalia, T. (2005) Proc. Nat. Acad. Sci. USA. 102: 3272-3277.

47. Tenchov, B.G., MacDonald, R.C., Siegel, D.P. (2006) Biophys. J. 91 : 2508-2518.

48. Bloch, K. (1983) CRC Crit. Rev. Biochem. 


\section{IR MOTION TRACKING AS A STANDARD INPUT DEVICE}

Michael Cimolini

Department of Computing Science, University of Alberta

\section{Abstract}

As our computing needs change and the availability of advanced input systems begin to increase we find that we are coming to the point where current interfaces are beginning to become outdated. Touch interfaces are becoming abundant, but there is only so much that one can do with a 2D input. This project tries to show that there is a potential for IR based interface systems to replace standard mouse input in current as well as future interfaces. Our results show that both the accuracy and robustness of the IR system designed herein are equivalent to that of standard mouse input.

\section{Introduction}

As the pricing of IR based tracking systems decrease, we find that they are now beginning to become affordable for consumer use. As the programs we use become more complex and our requirements from our systems increase we are starting to see a shift away from conventional mouse and keyboard input towards new input systems such as touch systems and motion tracking. In this experiment we look at the accuracy, robustness, and ease of use of an IR based tracking system for use as an input device. We compare the use of the IR system versus the mouse in two tests, the first with a program designed specifically for these tests, and the second with just the use of the standard Windows environment. The goals of these experiments are to show that an IR system could potentially replace mouse input in future interfaces and to show that the IR systems show the same reliability and accuracy as a standard mouse.

\section{Related Work}

There has been some work in this field, but as far as I have been able to determine there has been no work specifically using an IR tracking system to track rigid bodies, or work to determine how close in accuracy the IR system and mouse are. Work by Vlasic et. al. [3] has shown the use of a motion capture system for everyday use that shows a high degree of accuracy and reliability. This system uses ultrasonic sensors in combination with accelerometers to track motion. The novelty of this approach is that the ultrasonic sensors track the position of the accelerometers, giving them a reference frame and helping correct for drift. Jacanovic and MacKenzie [1] created a system using optical tracking to control mouse cursor movement. This system is similar in design to the IR desktop application in this experiment, however, it uses a printed marker which is tracked by a camera to control the system based on head position. Takase and Sawada [2] developed a system similar to the 3D brick breaker application of this experiment; however they used optical tracking to track a finger and hand as the control device for their system, instead of the IR based tracking of this experiment.

\section{System Design}

IR System

The IR tracking system used for these experiments was the natural point OptiTrack system using their Arena software system. This system uses six infrared cameras to track IR reflective markers. For this experiment all six cameras were calibrated and used, with a calibration area of approximately $2 \mathrm{~m} \times 3 \mathrm{~m} \times 2.5 \mathrm{~m}$. This calibration was completed using the inbuilt calibration tools in Arena. For the tracked object, three reflective markers were used. These markers were arranged on the supplied rigid body marker in a triangle pattern. This marker array was then attached to the back of a wrist brace so as to be worn on the right hand with the markers on the back of the hand. The Arena system software was used for motion tracking, with the streaming settings active. Only rigid body data was streamed which, for this experiment, meant that only data from the markers which were used to track the users hand were transmitted over the network.

\section{Network Application}

As Arena streams data through a network as a server, a client application was needed to capture and use this data. To this extent natural point created the NatNet SDK. This is a software development kit designed specifically to build client and server programs for use with the OptiTrack. The SDK is written for use in a $\mathrm{C}$ or $\mathrm{C}++$ developing environment. For this experiment the SDK was used to create a client which would capture and interpret the data from Arena. This client was programmed in $\mathrm{C}++$. The client program consists of two parts. The first interacts with the server, receiving data in a secure and reliable way through the use of inbuilt function in the NatNet SDK. The second part interprets this data from the Arena co-ordinates into screen co-ordinates. This data is then passed along to the user interactive program through either a socket connection as in the case of the 3D brick breaker game, or directly as in the case of the IR desktop application.

\section{D Brick Breaker}

This was the first of the two applications designed for the IR motion capture system. This application is very similar to the application developed by Takase and Sawada [2]. The premise and design for the game itself has been around for many years and is an adaptation of the original pong style games. The object of the game is to destroy all of the blocks while keeping the ball in the playing area. The game itself is programmed in Vizard, which is a development environment and extension for the Python programming 
language. As such this application was connected to the network application through the use of the Windows socket API so that data could be passed from the network application. The game uses the inbuilt physics and collision detection systems inherent in the Vizard libraries for physics simulation in game. The brick model, paddle model, and world model used in the game were modeled in 3DS Max and exported using a plug-in available on the WorldViz website, WorldViz being the developer of Vizard. These models were exported in the .DAE format for easy importing into the Vizard environment. The ball model used is a model that comes packaged with the Vizard program as part of the Duck Court example. The game itself consists of 72 randomly arranged blocks, with the blocks location being static between runs. The blocks were laid out in a way such that there was no overlap between blocks. Two nearly identical instances of the game were created, with the only difference between the two being the control method. One system was designed to be controlled with the IR tracking system and the other with the mouse. Mouse movement was mapped natively to the paddle, such that the movement of the mouse directly correlated to the movement of the paddle. For the IR controlled system, the mapping to paddle movement was much the same as with the IR desktop application; that being with reaching up mapping to upward movement of the paddle, down to downward movement, left to leftward movement and right to rightward movement. The sensitivity of the system was calibrated for the IR such that a motion of $0.75 \mathrm{~m}$ was necessary to move from either the left to the right of the screen, or from the top to the bottom. Score was calculated as the number of blocks destroyed minus the number of balls missed. A ball was considered missed when it had traveled passed the paddle in the negative z-axis, with the $z$-axis being perpendicular to the plane of the monitor.

\section{IR Desktop}

This was the second of two applications designed to take advantage of the IR motion capture system. This application is designed to control the mouse in a standard windows environment. This application was written in $\mathrm{C}++$, again using the NatNet SDK. It uses the Windows API to emulate mouse movement and mouse clicks. The application receives data from Arena through the network application. Movement of the mouse is controlled by movement of the tracked rigid body in the $x$ and $y$ coordinates of the screen, with the $x$-axis being measure perpendicular to the standing user, and the $y$-axis being measured parallel to the standing user, such that reaching up would move the mouse cursor up, left moving the cursor left, down moving the cursor down, and right moving the cursor right. The z-axis was measured perpendicular to the $x$ and $y$-axis, with reaching forward, away from the user, being the positive $z$-axis, and reaching behind being the negative z-axis. The z-axis was used to recreate mouse movements, with a movement of approximately $5 \mathrm{~cm}$ in the positive $z$-axis in a period of 0.5 seconds performing a left mouse click. For this experiment only left mouse clicks were used, however it would be possible to simulate right mouse clicks in a similar fashion. The sensitivity of the system was calibrated such that it would require a movement of $0.5 \mathrm{~m}$ to move the cursor from the top of the screen to the bottom of the screen, and a movement of $1 \mathrm{~m}$ would move the cursor from the left of the screen to the right of the screen.

\section{Experiment}

For the experiment, participants were randomly assigned to complete one of the two experiments first and then, after a 5 minute rest, were asked to complete the other experiment. For all participants, except for in one case, the same camera calibration results and rigid body marker calibration were used. For the one exception, the tracking volume had to be lowered as the height of the participant caused the markers to be lost while reaching towards the bottom of the screen. In total, twelve participants completed the experiment, with ten completing the IR desktop portion and the full twelve completing the $3 \mathrm{D}$ brick breaker portion. Throughout the experiment users were monitored for soreness or discomfort in their shoulders or arms, as these are noted as being related to gorilla arm syndrome, a common problem with interfaces requiring the arm to be raised for extended periods of time. Users were also asked to complete a survey at the end of the experiment rating their ability to continue to use the system for extended periods of time. This was used in a qualitative measure, not a quantitative measure, as a general assessment to determine the need for future experiments focusing on this issue.

\section{D Brick Breaker}

For this experiment two metrics were measured, these being time and score. Time was measured from when the first ball was launched to when the last brick was destroyed. It was used as a gross estimate more so then a solid metric as the time taken is variable based on the luck of bouncing of the ball. Also the system was designed to change the launch angle of the ball based on the position of the mouse cursor, which may have had some effect on time as well. Score was used to calculate the number of errors that being the number of times the ball passed off screen. This was calculated by subtracting the score from seventy two, the total number of blocks in the game. Participants were assigned to complete the game using either the IR tracking system or the mouse input first and then, after a three minute rest, were asked to complete the game a second time using the other input device. Users were assigned to these two groups in a pseudo random fashion, with six users being placed in each group. Each participant was given the same instruction at that start of each game, that being the objective of the game and the controls. Each participant was given two minutes to become familiar with the mouse controls, or four minutes with the IR controls. During the four minutes with the IR controls, the users were asked to touch each of the four corners of the play area to determine that the IR tracking system was properly calibrated and that they would indeed be able to reach the entire playing area. In cases where this was not possible, the game was restarted to recalibrate the tracking and position of the paddle. Due to a small glitch in the physics engine of the game, from time to time the ball 
would pass through the walls of the environment. When this occurred, a new ball was launched by clicking the mouse. This did not deduct points from the users score. During the participants playing of the IR controlled game, the mouse would be moved at random to adjust the position that the next ball would be fired at to emulate the way in which the ball would be fired in the mouse controlled game.

\section{IR Desktop}

For this experiment three metrics were measured, with these being time, errors, and number of clicks. Time and clicks were the major comparison for this experiment, with these being directly related to the speed and ease of use of the system. Time was measured from when the first instruction was read, until the last instruction had been completed. Number of clicks was recorded as the number of times the left mouse button was clicked in the case of the mouse, or as the number of forward presses made by the participant in the case of the IR system. Errors were measured as the number of times that the IR system lost tracking during the runs; this was used as a measure of robustness for the system. As with the first experiment, users were again assigned to two groups, with each group starting with one of the two control methods and then, after a ten minute rest, being asked to complete the experiment with the other input method. The reason for the extended rest in this case was due to the users having to complete the same set of tasks, so a ten minute time interval was used to try to cause the participants to forget the tasks they had completed in the first run. During this time period users were given a tour of the lab in hopes of aiding in causing them to forget the tasks they had completed. There were seven tasks that the users were asked to complete, all being standard Windows tasks. These tasks were read to the participants in order, with each subsequent task after the first only being read after the user had completed the previous task. The tasks were as follows: open an internet browser, open any bookmarked webpage, close the internet browser, open Notepad, close Notepad, open the Trashcan, and close the Trashcan. The point of these tasks was to cause the participants to interact with the largest screen area possible. To this extent the icon for the Trashcan was placed in the bottom right corner of the screen, and the icon for the internet browser was placed in the top right. As the user needed to reach the top left corner to close the internet browser, and the bottom left to open notepad, all corners of the screen were explored. The Notepad and Trashcan windows were set to open in the middle of the screen, allowing for exploring of the middle of the screen as well. For each trial, users were asked to begin with the cursor centered in the middle of the screen, such that the starting position would not be a confounding effect on the experiment, as well as allowing for a more thorough navigation of the centre of the screen. For both runs of the experiment, the screen resolution was lowered to $1440 \times 900$, with the experiment being run on a 22 " $16: 10$ aspect screen.

\section{Results}

\section{D Brick Breaker}

The statistics between the mouse and IR inputs were compared for both time and errors for this experiment, with the recorded results listed in Table I. of the appendix.

The mean time for the IR input was 04:21.7 minutes, and 03:51.7 minutes for the mouse input. For these results, we get a t-value of 1.306262 versus a t-critical value of 2.200985 , with 11 degrees of freedom. This gives a $p$-value of 0.218117 , meaning that there is no statistical difference between the times for the two input methods. A similar comparison of the errors leads us to a mean of 10.16667 for the IR and 9.916667 for the mouse. For this comparison we have a $t$-value of 0.166317 versus a $t$-critical value of 2.200985 with 11 degrees of freedom. This gives a $p$-value of 0.870925 , meaning that there is no statistical difference between the errors. For both of these measurements we find that we are unable to reject the null hypothesis that there is no difference between controlling this program with the IR system or with the mouse.

\section{IR Desktop}

The statistics between the mouse and IR inputs were compared for time, errors, and clicks for this experiment, with the recorded results listed in Table II. of the appendix.

The mean time for the IR input was 01:23.5 minutes and 00:21.1 minutes for the mouse input. For these results, we get a $t$-value of 6.722904 versus a $t$-critical value of 2.262157 with 9 degrees of freedom. This leads to a $p$-value of $8.63^{\wedge}-5$, meaning that there is a statistical difference between these means. For the clicks we get a mean of 32.8 for the IR input and 14.2 for the mouse input. This leads to a t-value of 9.1194 versus a t-critical value of 2.262157 with 9 degrees of freedom. This leads to a $p$-value of $7.66^{\wedge}-6$, meaning that there is a statistical difference in the mean value of clicks for the two input methods. The third statistic, errors, had a mean value of 0.6 for the IR input and 0 for the mouse input. This leads to a t-value of 2.25 versus a t-critical value of 2.262157 with 9 degrees of freedom. This leads to a p-value of 0.051003 , meaning that there is no statistical difference in the number of errors for the two input methods, but it is close.

\section{Conclusion}

A number of conclusions can be drawn from this experiment. The first is that when it comes to direct control of the cursor, an IR system can be just as reliable and accurate as a mouse. This is shown most notably in the 3D brick breaker experiment, where neither time needed to complete the game, nor number of errors showed any statistical difference for the two systems. This means that our null hypothesis that the two systems are the same holds true. As this experiment only showed differences in the ability to control the cursor we see that the accuracy of the two systems is highly comparable. The fact that there was also shown to be no statistical difference in the number of errors in the IR system versus the mouse in the IR desktop experiment shows that an IR system can be just as reliable as the mouse, even when used in a standard Windows environment for which the mouse was designed 
and optimized. From the IR desktop experiment we do, however, see a statistical difference in both time and number of clicks required. As we have noted previously that accuracy was no different for the mouse and IR system, we can attribute the difference in time to the clicking performed in the IR desktop experiment. This is mainly due to the design of the system. During the forward pressing motion of the hand to perform clicks in with the IR system, it was often noted to be the case that this would cause the mouse pointer to move, causing the participant to miss their intended target, leading to both an increase in clicks and, as a result, time taken to complete the experiment. Implications that can be drawn from these results are thus; an IR system can be as accurate and reliable as a mouse, though some conventions will need to be taken to augment the IR system with a more robust and accurate method of performing clicks than was presented in this research.

\section{Future Work}

\section{D Brick Breaker}

Any future work on the 3D brick breaker game would likely focus on depth perception. Through the trials it was noted that participants had difficulty tracking the exact location of the ball due to a lack of depth cues. Possible improvements to this system would be the inclusion of shadows for the ball, bricks, and paddle on all four walls of the environment allowing the users eyes to track these to determine relative depths. The other would be to modify the program to work with a 3D display which would then add depth perception to the program by the nature of the display.

\section{IR Desktop}

Future work for this system would focus on finding a better method to control clicking the left and right button. Methods that have been noted thus far would be using tracking of a second hand and having gestures from that hand control clicking, while having the main hand control movement. This would also allow for a form of multi touch, as two points could then be tracked. A second idea would be to add some sort of button system to the tracked hand or as a separate device to allow for clicking with the buttons of that device instead of through the use of gestures.

\section{General Program}

There is quite a bit that can be done as future work with the system in general. The first would be to add more tracking markers to the rigid body to increase the accuracy of tracking and to decrease errors. The second would be to look into possibilities to reduce the number of cameras used in tracking to decrease the size of the system, as well as decreasing the price. The third would be looking into the design of other games for the system as there is definitely some possibility for use of a system such as this for physical rehabilitation.

\section{References}

[1] Javanovic, R., \& MacKenzie, I. S., MarkerMouse: Mouse Cursor control using a head-mounted marker. Proceedings of the $12^{\text {th }}$ International Conference on Computers Helping People With Special Needs, ICCHP, 2010, pp.49-56. Berlin: Springer.

[2] Takase, H., \& Sawada, H., Gestural interface and the intuitive interaction with virtual objects. ICCAS-SICE, 2009, pp.3260-3263.

[3] Vlasic, D., Adelsberger, R., Vannucci, G., Barnwell, J., Gross, M., Matusik, W., \& Popović, J. (2007). Practical motion capture in everyday surroundings. In International Conference on Computer Graphics and Interactive Techniques (3rd ed., Vol. 26, Article 35). San Diego, California: ACM SIGGRAPH.

\section{Appendix}

TABLE I.

3D Brick Breaker Results

\begin{tabular}{|c|c|c|c|c|c|c|c|c|c|}
\hline \multicolumn{10}{|c|}{ IR } \\
\hline \multicolumn{3}{|c|}{ IRick Breaker } \\
\hline Time & Score & Bricks Left & Errors & First & Time & Score & Bricks Left & Errors & First \\
\hline $04: 21.8$ & 59 & 0 & 13 & $\mathrm{Y}$ & $02: 39.6$ & 67 & 0 & 5 & $\mathrm{~N}$ \\
\hline $06: 32.2$ & 63 & 0 & 9 & $\mathrm{Y}$ & $03: 06.3$ & 56 & 0 & 16 & $\mathrm{~N}$ \\
\hline $04: 10.8$ & 60 & 0 & 12 & $\mathrm{~N}$ & $04: 27.6$ & 60 & 0 & 12 & $\mathrm{Y}$ \\
\hline $05: 30.9$ & 54 & 0 & 18 & $\mathrm{~N}$ & $04: 23.4$ & 61 & 0 & 11 & $\mathrm{Y}$ \\
\hline $04: 55.1$ & 61 & 0 & 11 & $\mathrm{~N}$ & $03: 33.3$ & 66 & 0 & 6 & $\mathrm{Y}$ \\
\hline $04: 22.2$ & 62 & 0 & 10 & $\mathrm{Y}$ & $05: 31.7$ & 54 & 0 & 18 & $\mathrm{~N}$ \\
\hline $04: 02.7$ & 64 & 0 & 8 & $\mathrm{Y}$ & $03: 06.8$ & 64 & 0 & 8 & $\mathrm{~N}$ \\
\hline $04: 03.4$ & 67 & 0 & 5 & $\mathrm{Y}$ & $03: 04.8$ & 64 & 0 & 8 & $\mathrm{~N}$ \\
\hline $03: 52.7$ & 65 & 0 & 7 & $\mathrm{~N}$ & $03: 55.2$ & 62 & 0 & 10 & $\mathrm{Y}$ \\
\hline $03: 56.7$ & 62 & 0 & 10 & $\mathrm{~N}$ & $04: 45.0$ & 63 & 0 & 9 & $\mathrm{Y}$ \\
\hline $02: 41.3$ & 68 & 0 & 4 & $\mathrm{~N}$ & $03: 25.3$ & 66 & 0 & 6 & $\mathrm{Y}$ \\
\hline $03: 51.1$ & 57 & 0 & 15 & $\mathrm{Y}$ & $04: 20.8$ & 62 & 0 & 10 & $\mathrm{~N}$ \\
\hline
\end{tabular}

\section{TABLE II.}

IR Desktop Results

\begin{tabular}{|c|c|c|c|c|c|c|c|}
\hline \multicolumn{8}{|c|}{ Windows } \\
\hline \multicolumn{4}{|c|}{$\mathbf{I R}$} & \multicolumn{4}{|c|}{ Mouse } \\
\hline Time & Errors & Clicks & First & Time & Errors & Clicks & First \\
\hline 01:18.7 & 0 & 37 & $\mathrm{Y}$ & $00: 18.8$ & 0 & 19 & $\mathrm{~N}$ \\
\hline $02: 46.3$ & 2 & 39 & $\mathrm{~N}$ & $00: 32.8$ & 0 & 14 & Y \\
\hline 01:06.0 & 2 & 29 & $\mathrm{~N}$ & $00: 22.3$ & 0 & 12 & Y \\
\hline $01: 35.3$ & 1 & 32 & $\mathrm{Y}$ & $00: 15.6$ & 0 & 13 & $\mathrm{~N}$ \\
\hline $00: 54.8$ & 0 & 22 & $\mathrm{~N}$ & $00: 19.8$ & 0 & 15 & Y \\
\hline 01:06.6 & 0 & 36 & $\mathrm{~N}$ & $00: 20.8$ & 0 & 14 & $\mathrm{Y}$ \\
\hline 01:19.5 & 0 & 31 & $Y$ & $00: 20.3$ & 0 & 14 & $\mathrm{~N}$ \\
\hline 01:11.5 & 1 & 36 & $\mathrm{Y}$ & $00: 17.7$ & 0 & 13 & $\mathrm{~N}$ \\
\hline $00: 52.6$ & 0 & 24 & $\mathrm{~N}$ & $00: 16.7$ & 0 & 14 & Y \\
\hline $01: 43.8$ & 0 & 42 & $Y$ & $00: 25.9$ & 0 & 14 & $\mathrm{~N}$ \\
\hline
\end{tabular}




\section{INABILITY OF VIRAL SUPERANTIGENS TO INDUCE CD4-MEDIATED ISLET TRANSPLANT REJECTION}

Peter Ciechanowski

Department of Biological Sciences (Immunology), University of Alberta

\section{Abstract}

Superantigens have the ability to bypass the specific interactions of the MHC class II and T-cell receptor by binding outside of the peptide binding region and onto the V $\beta$ chain. This ability allows superantigens to stimulate a wide array of T-cell populations, irrespective of T-cell receptor (TCR) specificity. Research on bacterial and viral superantigens have demonstrated various outcomes ranging from superantigen dependent cellular cytoxicity (SDCC), rheumatoid arthritis, to superantigen stimulated T-cell clonal deletion and anergy. Due to its ability to proliferate a large population of T-cells, this paper asks whether superantigens have a role in islet transplant rejection. We used transgenic V 36 TCR mice specific for male ' $\mathrm{H}-\mathrm{Y}$ ' antigen as recipients to islet transplants. Donors comprised mice expressing endogenous superantigen specific for the V $\beta 6$ chain. Transplantation of these donor islets did not induce rejection. Recipients were also primed with ' $\mathrm{H}-\mathrm{Y}$ ' antigen to induce a CD4 effector memory T-cell population prior to islet transplantation. Even with primed recipients, donor islet transplants did not induce rejection by recipient transgenic mice.

\section{Introduction}

The immune system is an important evolutionary mechanism that provides humans the capability of fighting infectious agents such as bacteria and viruses (1). The immune system involves the use of particular sophisticated cells that perform two main duties: find and destroy the infectious agent while limiting damage to the host (2). While providing a great advantage over immunodeficient humans who are unable to protect themselves from foreign agents, the immune system does have drawbacks.

The immune system plays a major role in causing hypersensitivity and allergic reactions (3), as well as autoimmunity $(4,5)$. In relation to transplants, the host's immune system is a major player in determining the rejection or acceptance of the transplant. One would think that tissue or organ transplants between two individuals would not be a problem if both belong to the same species. But there are genetic variations between individuals that cause the recipient to recognize the genetically disparate molecules as foreign (6). As the immune system recognizes foreign antigens as dangerous to the host, the immune system detects the donor's organ or tissue as an infectious agent. Subsequently, this results in immunological transplant rejection (7).

The immune system is divided between innate and adaptive compartments. Although performing certain specific actions, it is now generally understood that these are not separate compartments. Both compartments communicate and function together and thus there is some overlap between the two (8). An important set of cells comprising the adaptive immune system is the T-cell.

\section{The Major Histocompatibility Complex}

The T-cell carries a T-cell receptor that is specific towards a particular foreign antigen. T-cell receptors (TCRs) interact with processed peptides presented on either the major histocompatibility complex (MHC) class I or class II molecules (9). The MHCs are highly polymorphic proteins that aid in presenting foreign peptides to the $\alpha \beta$ receptors of the TCR (10).

Active in almost all cell types, the MHC class I antigen presentation pathway involves presenting peptides at the cell surface. The peptides derive from the proteins synthesized in the cell at any time and thus enable CD8 T-cells to detect and kill any infected cell. The MHC class II presentation pathway is an important component of 'professional' antigen-presenting cells (APCs), such as B-cells (another adaptive immune system cell type) and macrophages. The peptides derive from exogenous proteins via endosomal compartments. The APCs present these exogenous peptides to CD4 T-cells, which then causes the CD4 T-cells to perform mechanism such as phagocytosis and macropinocytosis (11).

Because the MHC is highly polymorphic, both classes have the ability to bind and present a wide diversity of peptides. Through MHC restriction, TCRs on CD8 and CD4 $\mathrm{T}$-cells are restricted to recognizing peptides on host $\mathrm{MHC}$ molecules (12). Nonetheless, there are some TCRs that have an ability to bind to foreign MHC molecules with or without loaded peptides (13). Along with MHC restriction, the clonal selection theory argues that each T-cell expresses specificity to only one particular antigen (10).

\section{Transplant Rejection: Indirect and Direct Presentation}

Type- 1 diabetes is an autoimmune disease produced by $\mathrm{T}$ lymphocytes that selectively destruct insulinproducing pancreatic $\beta$-cells (14). A person's susceptibility and resistance to autoimmune disorders is mostly associated with polymorphisms of genes of the MHC. Islet transplantation has been used to treat type-1 diabetics, but its propensity for rejection has made it difficult to be a very successful treatment option.

There are two main pathways in the induction of transplant rejection. The first is 'direct' recognition where donor antigen presenting cells (APCs) stimulate host T-cells. The second is 'indirect' recognition where host APCs stimulate host T-cells (15). There is no concrete evidence which pathway is the most dominant in a given situation relating to a particular islet transplantation. On the other hand, observations have been made for each pathway suggesting their dominant role in islet transplant rejection.

There are three observations that argue 'direct' recognition is a major pathway in allograft rejection. First, in a primary allogeneic mixed lymphocyte reaction (MLR), direct stimulation is very prominent. Second, allograft 
survival may be sometimes prolonged with donor APC depletion. Third, donor MHCs are more important the minor antigens in producing graft rejection (15). Likewise, there are a couple of arguments explaining the importance of an 'indirect' response. First, this pathway alone may induce rejection. Second, in some instances where rejection involves both pathways, the 'indirect' pathway occurs much more rapidly than the 'direct' pathway (16). These two pathways, which involve two different kinds of APCs (one from the host and the other form the donor) is a fundamental feature of graft rejection that distinguishes it from other immune responses (16). Understanding the basis of transplant rejection will be important in understanding the rationale for choosing experimental models for evaluating the role of Superantigens in transplant rejection.

\section{Introduction to Superantigens}

A typical antigen has the capability to interact with 1 in $10^{4}$ to 1 in $10^{6} \mathrm{~T}$ cells. Normal antigens must be presented in a major histocompatibily complec $(\mathrm{MHC})$-restricted fashion as well as interact with a specific TCR. Superantigens are a class of immunostimulatory antigens of bacterial and viral origin (16). These are special antigens that do not require recognition by a specific TCR $\alpha / \beta$ heterodimer (16). Instead, the main requirement for recognition is superantigen binding $V \beta$ chain region of the TCR outside of the antigen binding site (17). As well, superantigens are presented on the MHC Class II molecule from an antigen presenting cell (APC). These particular antigens essentially act as a wedge between the TCR $\beta$ chain and the MHC class II $\alpha$ chain. Binding outside of the TCR $\alpha / \beta$ heterodimer means that there is a bypass from the normal mechanism for T-cell triggering by specific peptide/MHC complexes (18). Thus, superantigens have the capability of interacting with thirty percent of T-cells. This propensity for wide scale interaction with T-cells has given these special antigens several descriptors, mainly as minor histocompatibiliy antigens and mitogens. (19).

Superantigens gained notoriety in the first place due to their ability to produce superantigen dependent mediated cellular cytoxicity (SDCC). SDCC inevitably leads to toxic shock syndrome (TSS), which is a life-threatening intoxication produced by staphylococcal and streptococcal pyrogenic toxins (17). TSS is life threatening due to a massive cytokine release (e.g. TNF $\beta$, IL-2, and gamma interferon) leading to a variety of symptoms such as hypotension and multiorgan dysfunction (17). Staphylococcal enterotoxins are also associated with food poisoning and scaled skin syndrome due to proliferation of polyclonal T-cells (20).

Besides fates such as anergy, clonal deletion, or toxic shock syndrome, research is starting to investigate superantigen effects in autoimmune diseases. A human endogenous retrovirus HERV-K18 is believed to produce superantigens that play a role in developing rheumatoid arthritis (17). There has also been a link between an endogenous human retrovirus responsible for producing superantigens and primary biliary cirrhosis in recipients of liver transplants (21).

\section{Do Superantigens Play a Role in Islet Transplant Rejection?}

This research project addresses whether superantigens have the capability of inducing transplant rejection. To study the role of superantigens, this research focuses on the retrovirus called the 'Mouse Mammary Tumor Virus' (MMTV). MMTV can exist in two forms: germline integrated (endogenous MMTV) or as milk-transmitted infectious virus (exogenous MMTV) (22). All laboratory mouse strains contain endogenous MMTV, most of which have lost their infectious character of milk transmission (22).

In this experiment, transgenic mouse strains are used that carry only a specific $T$-cell receptor and V $\beta$ chain. As well, the donor will carry a known endogenous superantigen that has the capability to recognize the recipient's specific $V \beta$ chain. Using transgenic mouse strains eliminates variables that may induce transplant rejection other than a superantigen. Since the recipient $\mathrm{T}$-cell receptor only recognizes a specific antigen, then donor mismatching of the major histocompatibility complex cannot be a factor in transplant rejection. Through islet transplantation that carries an endogenous superantigen, if rejection does occur then it will be due to indirect presentation. Relating to the literature presented in the previous section on its role in autoimmune diseases, I expect the MMTV superantigen to produce a T-cell proliferative response that will lead to islet transplant rejection via the indirect presentation pathway.

\section{Materials and Methods \\ Mice}

Mice used in these experiments were: DBA/2NCR, B10 - [Tg]Marilyn - [KO] RAG2 - [KO] PD-1, B10 - [Tg]Marilyn [KO] RAG2, B6, BALB/c, and OT-2 mice. All mice received from either the $\mathrm{NCl}$-Frederick Cancer Research Institute or the Health Sciences Laboratory Animal Services.

\section{Media}

Culture media: $437.5 \mathrm{ml}$ IMDM (with NAHCO3), 50.0ml Fetal Bovine Serum (FBS), 5.0ml Penicillin/Streptomycin, $5.0 \mathrm{ml}$ Glutamine, $2.5 \mathrm{ml}$ Gentamycin, and $1.92 \mu \mathrm{l} \mathrm{beta-}$ mercaptoethanol. All ingredients must be added into an autoclaved bottle under a laminar flow hood with the use of a filter sterilizer.

Assay media: 487ml IMDM (with $\mathrm{NaHCO}$ ) and $13 \mathrm{ml}$ FBS into an autoclaved bottle under a laminar flowhood.

PBS $/ 0.1 \%$ BSA: $100 \mathrm{ml}$ PBS and $0.1 \mathrm{ml}$ Bovine Serum Albumin (BSA).

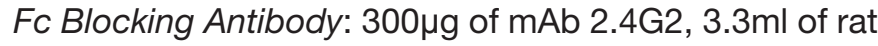
serum, $3.3 \mathrm{ml}$ of hamster serum, and $3.3 \mathrm{ml}$ mouse serum.

\section{Antibodies for Lymphocyte Staining}

Antibodies were diluted in $1 \%$ BSA, which was made from diluting $10 \%$ dialyzed BSA with PBS. Antibodies were diluted according to the appropriate concentration.

\section{Carboxyfluorescein Succinimidyl Ester (CFSE) Staining}

Took out the spleens from responder (cells tested for T-cell proliferation) and stimulator (cells used to induce T-cell proliferation). Responder spleens were placed in $\mathrm{PBS} / 0.1 \% \mathrm{BSA}$ while stimulator spleens went into 
culture media in separate $50 \mathrm{ml}$ conicals. All conicals were set in ice for the remainder of the experiment. Using a hemacytometer, $10 \mu \mathrm{l}$ was taken to count number of responder and stimulator cells. Responder cells were adjusted to a cell concentration range of $10-20 \times 10^{6}$ cells $/ \mathrm{ml}$ to make a volume of $10 \mathrm{ml}$. After setting the new concentration range, $1 \mathrm{ml}$ of responder cells were set aside to comprise the unlabelled CFSE responder cells. The unlabelled responder cells were set to a concentration $1 \times 10^{6} \mathrm{cells} / \mathrm{ml}$ in culture media. Simulator cells were set to a concentration of $3 \times 10^{6}$ cells $/ \mathrm{ml}$. Responder cells for labeling had $0.5 \mathrm{mM}$ CFSE added and mixed to $10 \mathrm{ml}$ of responder cell suspension. CFSE labeled responder cells incubated for 10 minutes at $37^{\circ} \mathrm{C}$. Adding $0.5 \mathrm{ml}$ FBS to the $10 \mathrm{ml}$ responder cell suspension quenched CFSE staining, which was then followed by 5 minutes incubation on ice. After incubation PBS/0.1\% BSA was added to the top of the tube and centrifuged $\left(4^{\circ} \mathrm{C}, 1200\right.$ RPM for 10 minutes). Cells were washed twice, with the last wash being with PBS. Labeled responder cells were FACS analyzed to confirm CFSE staining. After confirmed labeling, responder cells were counted once again with the hemacytometer and adjusted to a concentration of $1 \times 10^{6}$ cells $/ \mathrm{ml}$ with culture media. Stimulator mice were then irradiated (@1500 RADS) 1:20 seconds with the irradiator (irradiator manual was referred in order to set the correct time in relation to the decay factor). Culture wells were set up by producing a maximal volume of $2 \mathrm{ml}$ for each well. With the appropriate stimulators and responders, $1 \mathrm{ml}$ of each was added to the appropriate wells. Positive control for responder cells involved adding $1 \mathrm{ml}$ of $5 \mu \mathrm{g}$ Concanavalin A (ConA). Wells with no stimulator added had $1 \mathrm{ml}$ of culture media added instead. Culture wells incubated for 4 days at $37^{\circ} \mathrm{C}$, which were then FACS analyzed under the appropriate markers.

\section{Flow Cytometry}

The volume of Blocking Antibody, cells to be stained, and antibody were always the same to each other. Cell volumes of $25 \mu \mathrm{l}$ were placed in FACS tubes. Blocking Antibody $(25 \mu \mathrm{l})$ was then added to each FACS tube and subsequently vortexed and incubated at room temperature for 5 minutes. Then, $25 \mu$ l of diluted antibody was added, vortexed, and placed in a fridge for 15 minutes. Cells were washed with $3 \mathrm{ml}$ of PBS. After centrifugation, supernatant was dumped. Pellet was re-suspended in $300 \mu$ and vortexed. For peripheral blood, $20 \mu \mathrm{l}$ of heparin was added to $2 \mathrm{ml}$ conical tubes where about 15 to 20 drops of peripheral blood was collected and added to the $2 \mathrm{ml}$ conical tube. After, $30 \mu \mathrm{l}$ of mixed heparin and blood is placed into a FACS tube. $30 \mu$ l of Blocking Antibody and diluted antibody were added, respectively. After 15 minutes in the fridge, $3 \mathrm{ml} \mathrm{RBC}$ lysis buffer is added to each FACS tube and centrifuged ( $4^{\circ} \mathrm{C}, 1200 \mathrm{RPM}, 10$ minutes). After centrifugation the supernatant was dumped and the pellet re-suspended in $300 \mu \mathrm{l}$. FACS dot plots were analyzed using CellQuest.

\section{Histology}

The 'Heritage Research and Innovation Facility' laboratory performed histology of mice kidney capsules.
Kidney capsules were stained with hematoxylin and eosin.

\section{Islet Transplantation:}

A concentration of $195 \mu \mathrm{g} / \mathrm{kg}$ of streptozotocin was added to recipient mice through intraperitoneal injection three days prior to the day of transplantation. A 'ONE TOUCH ULTRA 2' blood glucose reader with a calibration code of ' 25 ' monitored blood glucose levels. Two successive readings of greater than $15 \mathrm{mmol} / \mathrm{L}$ from the glucose reader confirmed the recipient mouse as diabetic. On the day of transplantation, two donor mice were used for one recipient (ratio of 2:1). After transplantation, blood glucose readings were taken to confirm success of islet transplantation (a reading of less than $10 \mathrm{mmol} / \mathrm{L}$ ). After confirmation of islet transplant, blood glucose readings were taken periodically. FACS analysis of recipient peripheral blood was taken after 10 and 16 days post-transplantation.

\section{Priming Recipient Mice:}

B6 male splenocytes were mixed with PBS. Splenocytes were counted with a hemacytometer and cell concentration was adjusted to $12 \times 10^{6}$ cells $/ 1 \mathrm{ml}$. Recipient mice were intraperitoneal injected with $0.250 \mathrm{ml}$ of $\mathrm{B} 6$ male splenocytes. Eleven days after injection, peripheral blood of recipient mice was analyzed under FACS analysis to confirm successful priming. After confirmation, primed recipient mice received islet transplants.

\section{Results}

DBA-2 and BALB/C Superantigens Are Capable of Stimulating Monoclonal Naïve T-cell Populations:

DBA-2 mouse contains an endogenous MMTV superantigen that can recognize and stimulate T-cells with a V $\beta 6$ T-cell receptor chain. Marilyn mice contain a monoclonal T-cell population as well as the V $\beta 6$ T-cell receptor chain. Before performing any experiments with donor DBA-2 islet cells and recipient Marilyn, it must first be established that DBA-2 superantigen has the capability of stimulating the Marilyn monoclonal T-cell population. OT-2 mice also have a monoclonal T-cell population that can recognize only Ovalbumin (OVA) as well as a VB5 T-cell receptor chain. BALB/c mice carry an endogenous MMTV strain that can recognize the $V \beta 5$ chain, so the BALB/c OT-2 experimental model was used as a comparison to the DBA-2 - Marilyn model. Because studies have shown that some superantigens may cause clonal anergy, deletion, or both after T-cell stimulation, Marilyn PD-1 KO mice are used. A mouse deficient in PD-1 will have T-cells that will not have inhibition of activation upon stimulation and will proliferate at much higher rates than wild type Marilyn.

Female Marilyn and OT-2 mouse splenocytes were mixed with irradiated splenocytes of different stimulator groups. Female Marilyn responder splenocytes were mixed with either: B6 female (no antigen), B6 male (male ' $\mathrm{H}-\mathrm{Y}$ ' antigen) or DBA-2 female (superantigen) stimulators. OT-2 responder splenocytes were mixed with either: B6 (no antigen), OVA (antigen), or BALB/c (superantigen). Controls for both experimental models was adding no stimulators (negative control) and adding ConA (positive control). ConA is a mitogen that binds to T-cell receptors and thus 
stimulates proliferation.

This 'in vitro' experiment showed that responder T-cells from both female Marilyn PD-1 KO and OT-2 mice were recognized by their respective stimulator superantigens: DBA-2 and BALB/c, respectively. ConA stimulator showed the highest $\mathrm{T}$-cell proliferation rates for both responder mice. Proliferation rates for antigen stimulated were similar in comparison to both responders, with $22 \%$ for B6 male and $28 \%$ for OVA female. On the other hand, proliferation rates for superantigens varied greatly between the two responders. With DBA-2, the proliferation rate was $47 \%$ while for BALB/c it was $8.15 \%$. This showed that different superantigens recognizing different $V \beta$-chain TCRs have different capabilities in stimulating monoclonal T-cell proliferation.

A problem with performing the 'in vitro' experiment was that absolute numbers of gated CD90.2 ${ }^{\mathrm{HI}} \mathrm{CD} 4^{\mathrm{HI}} \mathrm{T}$-cells varied greatly. An example was with $\mathrm{CD} 90.2^{\mathrm{HI}} \mathrm{CD} 4^{\mathrm{HI}} \mathrm{T}$-cells gated for experimental group with no stimulators added. For Marilyn PD-1 KO, there were sixty-two gated events while for OT-2 there were 934 gated events. As well, I performed this same experiment with Marilyn mice and there was little to no lymphocytes present in all experimental groups. This variability in T-cell population may be attributed to steps in the experimental process, which may have resulted in loss to T-cell numbers.

First, I used the hemacytometer in counting splenocytes for adjusting appropriate cell concentrations. This counting process accounts for all cells (except RBCs) and not only T-cell populations. It was possible that I did not accurately adjust splenocyte populations to the correct concentrations and this might have overall affected T-cell population concentration numbers.
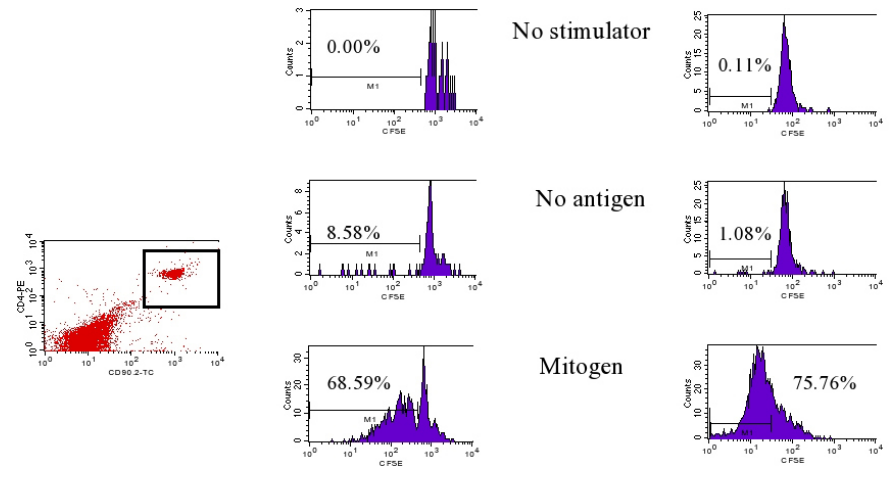

No antigen

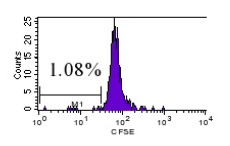

Mitogen
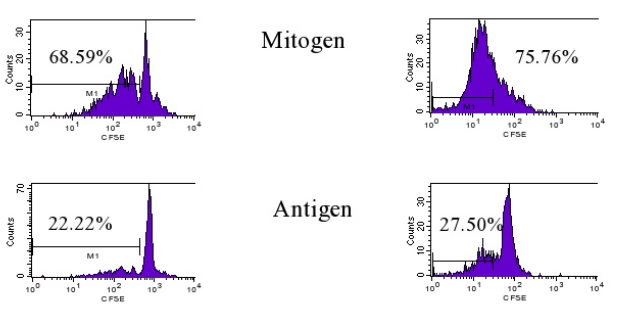

Antigen
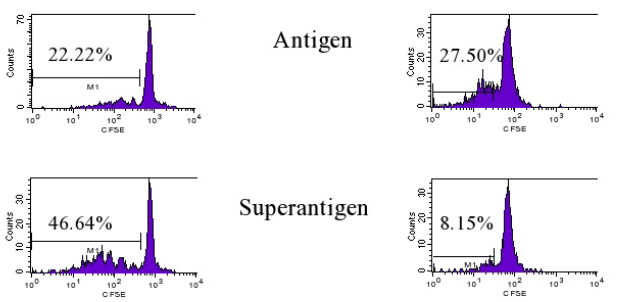

Superantigen

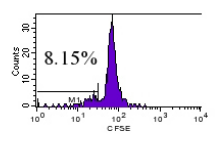

Figure 1. T-cell proliferation histogram analysis of $1 \times 10^{6} \mathrm{CFSE}$ labeled responder splenocytes with $3 \times 10^{6}$ irradiated stimulator splenocytes. CFSE was added with a concentration of $0.5 \mathrm{mM}$ and analysis was analyzed after 4 days of incubation at $37^{\circ} \mathrm{C}$. Data was gated on $\mathrm{TCR}^{\mathrm{HI}} \mathrm{CD} 90.2^{\mathrm{HI}}$ cell populations through dot plot analysis. Marilyn PD-1 KO female and OT-2 responder female splenocytes were used on the left and right columns, respectively. No stimulators contained only responder splenocytes. No antigen stimulators were B6 female (left) and B6 male (right). Antigen stimulators contained B6 male (right) and OVA female (left). Superantigen stimulators contained DBA-2 female (right) and BALB/c female (left).
Second, I used a vacuum with a small pipet to remove supernatants after centrifuging and I might have involuntarily removed a significant portion of the T-cell population. These two factors (incorrect concentration adjustments and incorrect removal of supernatant) might have contributed to adding less than $1 \times 10^{6}$ responder cells.

From the in vitro experiment, it was shown that superantigens have the ability to stimulate monoclonal T-cell populations to proliferate. This being demonstrated, the next process was to study whether these superantigen
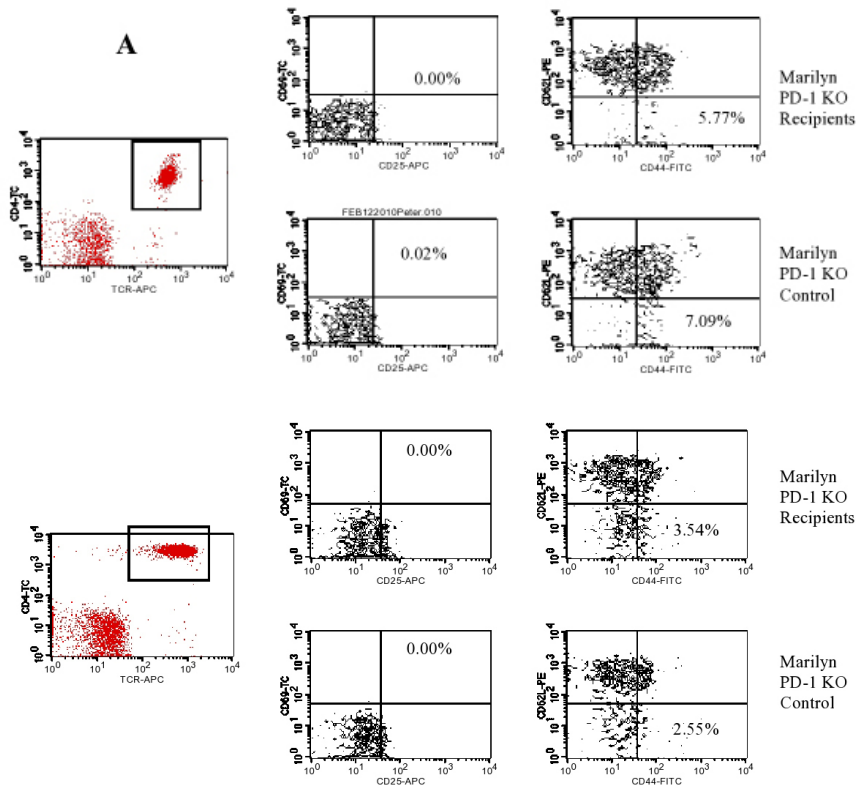
Recipients
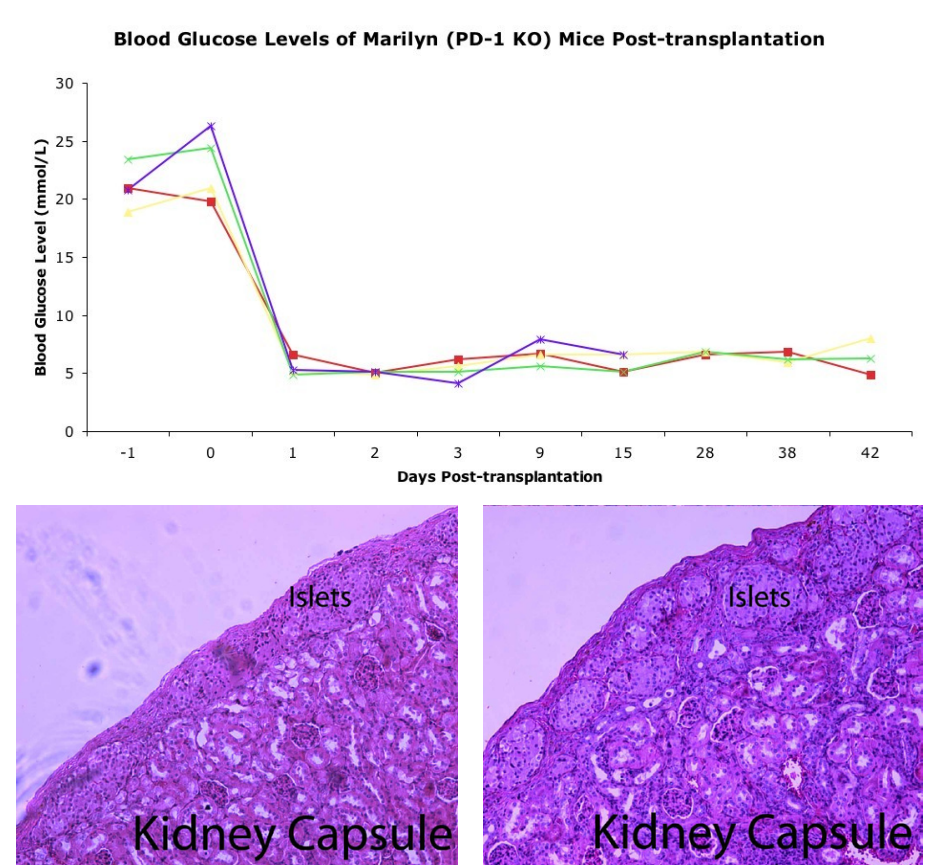

Figure 2. Marilyn PD-1 KO female recipients $(n=4)$ received DBA-2 female islet transplants and were monitored for rejection. $A$, Dot plot analysis for $\mathrm{CD} 64^{\mathrm{HI}}$ $\mathrm{CD}^{2} 5^{\mathrm{HI}}$ and $\mathrm{CD} 62 \mathrm{~L}^{\mathrm{LO}} \mathrm{CD} 44^{\mathrm{HI}}$ cells measured 10 days post-islet transplantation. Both dot plots were gated on TCR ${ }^{\mathrm{HI}} \mathrm{CD}^{\mathrm{HI}}$. Marilyn PD-1 KO female recipients $(\mathrm{n}=4)$ located above and Marilyn PD-1 KO female control located below $(\mathrm{n}=4)$. $B$, Dot plot analysis for $\mathrm{CD} 64^{\mathrm{HI}} \mathrm{CD} 25^{\mathrm{HI}}$ and $\mathrm{CD} 62 \mathrm{~L}^{\mathrm{LO}} \mathrm{CD} 44^{\mathrm{HI}}$ cells measured 16 days post-islet transplantation. Both dot plots were gated on TCR ${ }^{\mathrm{HI}} \mathrm{CD} 4^{\mathrm{HI}}$. Marilyn PD-1 $\mathrm{KO}$ recipients $(\mathrm{n}=4)$ located above and Marilyn PD-1 KO control located below $(\mathrm{n}=3)$. C, Blood glucose levels measured of recipient Marilyn PD-1 KO mice $(\mathrm{n}=4) . D$, Hematoxylin and Eosin staining of kidney capsules of recipient Marilyn PD-1 KO female mice $(n=3) 42$ days post-transplantation. 
stimulated monoclonal T-cell populations were capable of causing islet transplant rejection.

Recipient Marilyn PD-1 KO mice received DBA-2 female islet transplants after becoming streptozocin-induced diabetic. Two DBA-2 donors were added for every recipient mouse.

Dot plot analysis of recipient Marilyn PD-1 KO mice showed no comparable difference of activation markers (CD69 $\left.{ }^{\mathrm{HI}} \mathrm{CD}^{2} 5^{\mathrm{HI}}\right)$ for gated $\mathrm{TCR}^{\mathrm{HI}} \mathrm{CD}^{\mathrm{HI}}$ cells for both 10 and 16 days post-transplantation. Population percentage for CD62L ${ }^{\mathrm{LO}} \mathrm{CD}_{4} 4^{\mathrm{HI}}$ in both 10 and 16 days posttransplantation did not show any marked differences between the experimental and control group. Blood glucose readings post-transplantation showed that that recipient mice did not become diabetic, as they did not exceed two consecutive readings greater than $15 \mathrm{mmol} / \mathrm{L}$. Histology of kidney capsules stained with hematoxylin and eosin did not show any presence of lymphocyte infiltration.

Although in vitro analysis showed that superantigen was capable of stimulating a naïve monoclonal T-cell population, DBA-2 superantigen does not induce recipient immune rejection of islet transplants. The next process would be to examine if a recipient with a primed monoclonal T-cell population may induce islet transplant rejection.

\section{Priming Recipient Marilyn and Marilyn PD-1 KO Mice}

Since it was not known what fate naïve T-cells would have after stimulation by superantigens, B6 male carrying ' $\mathrm{H}-\mathrm{Y}$ ' antigen was used in the priming process. Approximately $3 \times 10^{6}$ splenocytes per $0.250 \mathrm{ml}$ were injected into each female Marilyn and Marilyn PD-1 KO recipient mouse via IP injection. After 11 days post-B6 male splenocyte injection, female Marilyn and Marilyn PD-1 KO mice were analyzed through flow cytometry to detect any presence of effector memory T-cells (CD62L LO CD44 H').

Marilyn mice injected with B6 male splenocytes showed an effector memory T-cell population percentage of $1.33 \%$, $0.82 \%$, and $0.69 \%$, respectively. These percentages were higher than the control Marilyn, which was $0.10 \%$. Likewise, B6 male splenocyte injected Marilyn PD-1 KO female mice showed a percentage of $2.68 \%$, while control female Marilyn PD-1 KO had a percentage of $0.03 \%$.

One difficulty I had was deciding which injected mice displayed a sufficient percentage of effector memory T-cell population when compared to the controls. I found percentages of $1.26 \%$ and $2.68 \%$ to be sufficiently larger than the controls to warrant their use in the next islet transplant experiment. On the other hand, the two Marilyn mice with percentages of $0.76 \%$ and $0.59 \%$ were difficult to determine whether these mice were truly primed or not. Future statistical analysis of data with an 'unpaired t-test' did indeed show that the percentages between control and experimental groups was not significant. As well, even though histology did not show any T-cell infiltration, I did not have control groups to compare with the experimental mice.

With an experimental group deemed to be primed at the time. The next phase was to perform DBA-2 female islet transplants to both the primed Marilyn and Marilyn PD-1 $\mathrm{KO}$ mice.
Donor DBA/2NCR Islet Transplants Do Not Induce Transplant Rejection in Primed Marilyn and Marilyn PD-1 KO Recipient Mice

Due to no difference of both $\mathrm{CD} 69^{\mathrm{HI}} \mathrm{CD} 25^{\mathrm{HI}}$ and CD62 $\mathrm{L}^{\mathrm{LO}}$ CD44 ${ }^{\mathrm{HI}}$ cells in unprimed female Marilyn PD-1 KO, this experiment involved the use of Marilyn and Marilyn PD-1 $\mathrm{KO}$ mice primed by male ' $\mathrm{H}-\mathrm{Y}$ ' antigen. Having T-cell receptors specific for the male ' $\mathrm{H}-\mathrm{Y}$ ' antigen, this ensured

Figure 4. $A$, Primed female Marilyn and Marilyn PD-1 KO recipients received female DBA-2 islet transplants and were screened for activation markers (CD69H ${ }^{H}$ CD25 $\left.{ }^{H I}\right)$ and an effector memory T-cell population (CD62L L ${ }^{\mathrm{LO}} \mathrm{CD}_{4} 4^{\mathrm{H}}$ ) 11 days post-transplantation. Dot plot analyses were gated on TCR ${ }^{H}$ $\mathrm{CD} 4^{\mathrm{HI}}$. The average percent for activation markers (CD69 ${ }^{\text {HI }}$ CD25 ${ }^{\text {HI }}$ ) and effector memory T-cell populations (CD62L Lo $\mathrm{CD} 44^{\mathrm{H}}$ ) was calculated for female recipient Marilyn PD-1 KO $(n=1)$ and Marilyn $(n=2)$ mice. Percentages of control female Marilyn ( $n=1)$ and Marilyn PD-1 $\mathrm{KO}(\mathrm{n}=1)$ is shown (right). $B$, Blood glucose levels were taken periodically post-islet transplantation to monitor for signs of islet transplant rejection.

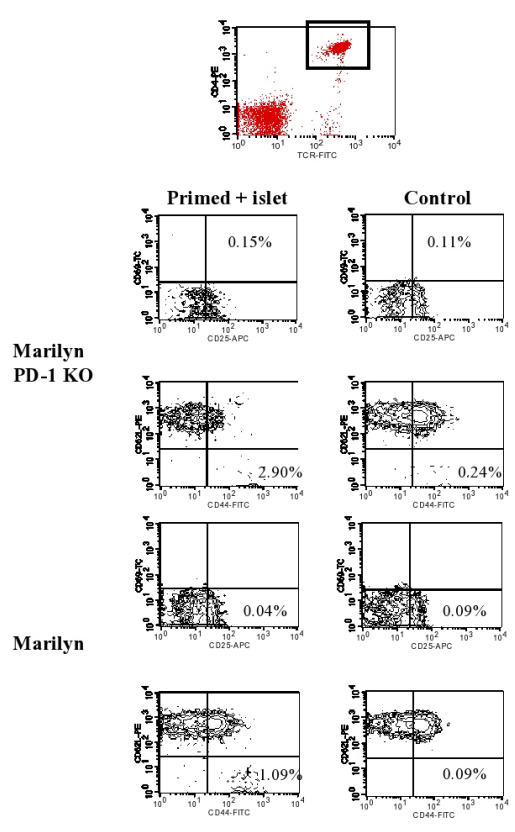

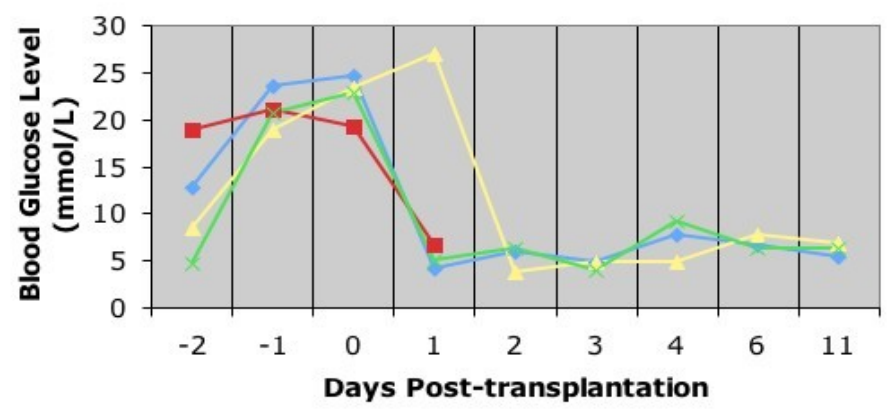
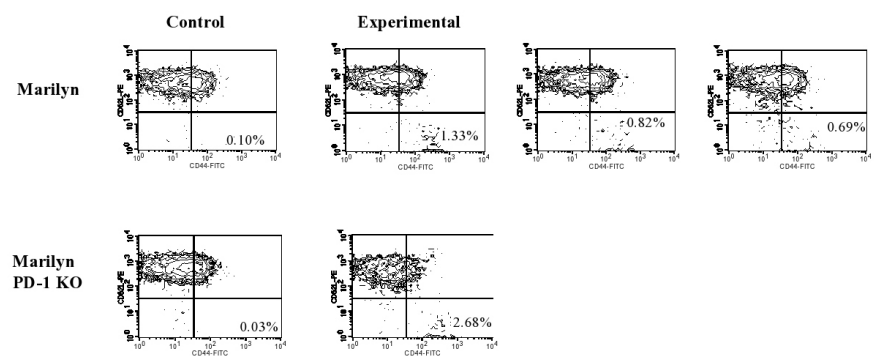

Figure 3. $A$, Female Marilyn mice $(n=3)$ were injected with $3 \times 10^{6} B 6$ male splenocytes. Representative plots show percent of effector memory T-cell population for control (left, $n=1$ ) and injected B6 male splenocytes (right) after 11 days IP injection. Female Marilyn PD-1 KO mice $(n=1)$ were injected with $3 \times 10^{6} \mathrm{~B} 6$ male splenocytes. Representative plots show percent of effector memory T-cell population for control (left, $n=1$ ) and injected B6 male splenocytes (right) after 11 days IP injection. 
that the priming process produced a sufficient number of memory effector T-cells that could be tested for islet rejection upon superantigen stimulation. Primed Marilyn and Marilyn PD-1 KO received DBA-2 islet transplants and were screened for activation markers (CD69 ${ }^{\mathrm{HI}}$ CD25 $\left.5^{\mathrm{HI}}\right)$ and an effector memory T-cell population (CD62L ${ }^{\mathrm{LO}} \mathrm{CD}^{\mathrm{C}} 4^{\mathrm{H}}$ ) 11 days post-transplantation.

Analysis of primed Marilyn recipient mice $(n=2)$ showed no large differences of activation markers $(0.04 \%)$ and effector memory T-cell populations (1.09\%) when compared with Marilyn control mouse $(n=1)$, which had $0.09 \%$ for both populations. Activation markers for primed Marilyn PD-1 KO mouse $(n=1)$ was $0.15 \%$ and this did not deviate far from control $(0.11 \%, n=1)$. On the other hand, effector memory T-cell populations for Marilyn PD-1 KO mouse $(n=1)$ had a slightly greater percentage $(2.90 \%)$ than control (0.24\%). Even with slight differences in effector memory populations for both experimental groups in comparison to control, primed recipient mice did not reject DBA-2 female islets, because blood glucose levels did never exceeded $15 \mathrm{mmol} / \mathrm{L}$.

\section{Discussion}

The main question asked in this research project was whether superantigens have a role in stimulating islet transplant rejection. The main conclusion reached is that superantigens do not mediate islet transplant rejection through CD4 T-cells, but the data obtained does not indicate as to what processes occur to induce tolerance of the DBA-2 superantigen.

Addressing this question involved asking three smaller questions: (1) if superantigens have the ability to stimulate monoclonal T-cell populations, (2) if superantigens have the ability to stimulate recipient naïve CD4 T-cells to result in transplant rejection, and (3) if superantigens have the ability to induce islet transplant rejection in primed recipient mice.

The first experiment involved culturing two different responder cells (female Marilyn PD-1 KO and female OT-2 mice) to different stimulator cells. This in vitro experiment did indeed show that female DBA-2 and BALB/c carrying superantigens could effectively stimulate proliferation of female Marilyn PD-1 KO and OT-2 monoclonal T-cell populations, respectively. This demonstrated that the OT-2 and $B A L B / c$ superantigens recognized the respective V $\beta$-chains of the Marilyn and OT-2 mice (V $\beta 6$ and V $\beta 5$, respectively). Proliferation data on female Marilyn mice were not successfully obtained and it would have been useful to compare proliferation rates between Marilyn and Marilyn PD-1 KO mice.

Recent research on superantigens has started to focus the role of $\mathrm{V} \alpha$-chains in being recognized by superantigens (23). As such, a useful future experiment would be to perform the same in vitro experiment (involving a V $\beta 6$ chain and DBA-2 superantigen) but with a different library of $\mathrm{V} \alpha$ chains. If there are significant differences in T-cell proliferation rates for some of the $\mathrm{Va}$ chains, then it may indicate a role of Va-chain and superantigen interaction. As well, mice carrying these Va-chains may subsequently be used in islet transplant experiments and see if rejection occurs.
The second experiment used female Marilyn PD-1 KO mice as recipients for female DBA-2 islet transplants. Presence effector markers were similar to that of control and recipients did not become diabetic. In the third experiment, Marilyn and Marilyn PD-1 KO mice primed with male ' $\mathrm{H}$ $Y^{\prime}$ ' antigen did not become diabetic when transplanted with female DBA-2 islets. Results obtained for activation markers were similar to unprimed recipients that were gated on $\mathrm{TCR}^{\mathrm{HI}} \mathrm{CD} 4^{\mathrm{HI}} \mathrm{T}$-cells. On the other hand, there was a slight increase (about 1-2\%) of effector memory T-cell populations in comparison to the control group.

Variable times of $\mathrm{T}$-cell proliferation by superantigens have been shown depending on MHC haplotype (24). In $\mathrm{H}-2^{\mathrm{b}}$ mice, for instance, peak proliferation generally occurred between days 6 and 8 (24). For MMTV, proliferation of T-cells peaked at 2 to 3 days (25). Because it is not well known how MHC haplotype will affect MMTV superantigen stimulated T-cell proliferation, screening for markers should be performed in the first ten days of transplantation. Data obtained on these two days could then be used to compare in later screenings to determine if: (1) T-cell populations actually proliferated in vivo in the first place, (suggesting clonal deletion in screenings 10 and 16 days post-transplantation) and (2) presence of activation markers if proliferation was present.

It is fairly established that superantigens have a propensity to stimulate polyclonal T-cell proliferation. The problem arises when addressing what particular effect this proliferation produces. Most of the research performed on superantigens has been done on the pyrogenic toxin superantigen family. This family includes the staphylococcal enterotoxins (SE)A through I (except F), staphylococcal toxic shock syndrome toxin-1 (TSST-1), streptococcal superantigen (SSA), and streptococcial pyrogenic exotoxins (SPE)A-C and -F $(26,27,28)$. In vivo studies performed with these pyrogenic toxins revealed $\mathrm{T}$-cells that become either anergic or deleted after proliferation. It is suggested that the fate of proliferated T-cells has more to do with what specific TCR V $\beta$-chain interacts with a particular superantigen (29). As well, other studies have explained that an in vivo response to superantigens gives rise to T-cells that are anergic if they survive deletion $(30,31,32)$.

Relating to the primed Marilyn and Marilyn PD-1 KO experiment, it is unknown whether the small presence of effector memory $\mathrm{T}$-cells in the primed recipient mice are anergic. To test if this population is anergic, effector memory T-cells from the primed recipient mice should be isolated and placed with two different stimulators: male ' $\mathrm{H}-\mathrm{Y}$ ' antigen and female DBA-2 superantigen. Another experiment that should be performed in the future is priming Marilyn and Marilyn mice with DBA-2 female mice prior to female DBA-2 islet transplantation.

Marilyn mice reject $\mathrm{B} 6$ male islet transplants due to having a T-cell receptor specific to only male ' $\mathrm{H}-\mathrm{Y}$ ' antigen (33). As such, it would also be useful to examine the effect of superantigen male islet transplants and see whether there would be faster, slower, or no rejection. 


\section{Acknowledgements}

I would like to thank Colin Anderson for letting me work in his lab and to obtain research experience under a wonderful working environment. I would also like to thank him for his intellectual guidance in my direct research topic. For all of my questions and troubleshooting I am grateful to Haide Razavy, Christa Smolarchuk, David Al-Adara, Catherine Ewen, and Raju Thangavelu.

\section{References:}

1. Cooper, M. D., and M. N. Alder. 2006. The evolution of adaptive immune systems. Cell 124: 815-822.

2. Hantash, B. M., L. Zhao, J. A. Knowles, and H.P. Lorenz. 2008. Adult and fetal wound healing. Front Biosci 13:51-61.

4. Holgate, S. T., and R. Polosa. 2008. Treatment strategies for allergy and asthma. Nat Rev. Immunol.

5. Brimkow, M. E., E. W. Jeffrey, K. A. Hjerrild, B. Paeper, L. B. Clark, S. A. Yasayko, J. E. Wilkinson, D. Galas, S. F. Ziegler, and F. Ramsdell. 2001. Disruption of a new forkhead/winged-helix protein, scurfin, results in the fatal lymphoproliferative disorder of the scurfy mouse. Nat Genet 27:68-73.

6. Lahl, K,. C. Loddenkemper, C. Drouin, J. Freyer, J. Arnason, G. Eberl, A. Hamann, H. Wagner, J. Huehn, and T. Sparwasser. 2007. Selective depletion of Foxp3+ regulatory $T$ cells induces a scurfy-like disease. J Exp Med 204:57-63.

7. Roopenian, Derry; Choi, Evan Young; Brown, Aaron. Immunological Reviews, Dec2002, Vol. 190 Issue 1, p86.

8. Borghesi, L., and C. Milcarek. 2007. Innate versus adaptive immunity: a paradigm past its prime? Cancer Res 67:3989-3993.

9. Sagerstrom, C.G., E. M. Kerr, J. P. Allison and M. M. Davis. 1993. Activation and differentiation requirements of primary T cells in vitro. Proc Natl Acad Sci U S A 90:89878991.

10. Felix, N. J., and P. M. Allen. 2007. Specificity of T-cell alloreactivity. Nat Rev Immunol 7:942-953.

11. Stefanová, I., Dorfman, J., \& Germain, R. (2002). Self-recognition promotes the foreign antigen sensitivity of naive T lymphocytes. Nature, 420(6914), 429.

12. Jensen, P. (2007). Recent advances in antigen processing and presentation. Nature Immunology, 8(10), 1041-1048.

13. Gras, S., Kjer-Nielsen, L., Burrows, S., McCluskey, J., \& Rossjohn, J. (2008). T-cell receptor bias and immunity. Current Opinion in Immunology, 20(1), 119-125.

14. Burnet, F. M. 1959. The Clonal Selection Theory of Acquired Immunity. Vanderbilt University Press, Nashville, TN.

15. Conrad, B., \& Weissmahr, R. (1997). A human endogenous retroviral superantigen as candidate autoimmune gene in type I diabetes. Cell, 90(2), 303.

16. Gould, D., \& Auchincloss, H. (1999). Direct and indirect recognition: the role of $\mathrm{MHC}$ antigens in graft rejection. Immunology Today, 20(2), 77-82.

17. Li, H., Llera, A., Malchiodi, E., \& Mariuzza, R. (1999). THE STRUCTURAL BASIS OF T CELL ACTIVATION BY SUPERANTIGENS. Annual Review of Immunology, 17(1),
435.

18. Olivera J. F., L., A., Debruyne, \& Bishop K., D. (1996). T Cell Receptor (TCR) Repertoire in Alloimune Responses. Intern Rev. Immunol. 13, 187-207.

19. Cauley, L., Miller, E., Yen, M., \& Swain, S. (2000). Superantigen-induced CD4 T cell tolerance mediated by myeloid cells and IFN-gamma. Journal Of Immunology (Baltimore, Md.: 1950), 165(11), 6056-6066.

20. Sicat, J., Sutkowski, N., \& Huber, B. (2005). Expression of human endogenous retrovirus HERV-K18 superantigen is elevated in juvenile rheumatoid arthritis. Journal of Rheumatology, 32(9), 1821-1831.

21. Lizhe, X., Zhiwei, S., Linsheng, G., Fodera, B., Keogh, A., Joplin, R., et al. (2003). Does a betaretrovirus infection trigger primary biliary cirrhosis?. Proceedings of the National Academy of Sciences of the United States of America, 100(14), 8454.

22. Luther, S., A., \& Acha-Orbea, H. (1996). Immune response to mouse mammary tumor virus. Current Opinion in Immunology, 8(4), 498-502.

23. Pumphrey, N., Vuidepot, A., Jakobsen, B., Forsberg, G., Walse, B., \& Lindkvist-Petersson, K. (2007). Cutting edge: Evidence of direct TCR alpha-chain interaction with superantigen. Journal Of Immunology (Baltimore, Md.: 1950), 179(5), 2700-2704.

24. Hayden, K., Tough, D., \& Webb, S. (1996). In vivo response of mature $\mathrm{T}$ cells to Mlsa antigens. Longterm progeny of dividing cells include cells with a naive phenotype. Journal Of Immunology (Baltimore, Md.: 1950), 156(1), 48-55.

25. Acha-Orbea, H., \& MacDonald, H. (1995). Superantigens of mouse mammary tumor virus. Annual Review Of Immunology, 13459-486.

26. Bohach G., A., Fast D.J., Nelson R., D., Schlievert, P., M. 1990. Staphylococcal and streptococcal pyrogenic toxins involved in toxic shock syndrome and related illnesses. Crit. Rev. Microbiol. 17:251-272.

27. Betley M., J., Borst D., W., Regassa L., B. 1992. Staphylococcal enterotoxins, toxic shock syndrome toxin and streptococcal pyrogenic exotoxins: a comparative study of their molecular biology. Chem. Immunol. 55:1-35.

28. Bohach, G., A. 1997. Staphylococcal enterotoxins B and C. In Superantigens: Molecular Biology, Immunology and Relevance to Humans Disease, ed. DYM Leung, BT Huber, PM Schlievert, Marcel Dekker Inc. NY, pp. 167-198.

29. Rellahan, B. L., Jones, L. A. Kruisbeek, A. M., Fry, A. M. \& Matis, L. A. 1990. In vivo induction of anergy in peripheral ${\mathrm{V} \beta 8^{+}}^{+} \mathrm{T}$ cells by staphylococcal enterotoxin $\mathrm{B}$. J. Exp. Med. 172:1091.

30. Sundstedt, A., Holden, I., Hansson, J., Hedlund, G., Kalland, T. and Dohlsten, M. 1995. Superantigen-induced anergy in cytotoxic CD8 ${ }^{+}$T cells. J. Immunol. 154:6306.

32. Macdonald, H. R., Baschieri, S. and Lees, R. K. 1991. Clonal expansion precedes anergy and death of $\mathrm{V} \beta 8^{+}$ peripheral $T$ cells responding to staphylococcal enterotoxin B in vivo. Euro. J. Immunol. 21:1963.

33. Chan, W., Razavy, H., \& Anderson, C. (2008). Differential susceptibility of allogeneic targets to indirect CD4 immunity generates split tolerance. Journal Of Immunology (Baltimore, Md.: 1950), 181(7), 4603-4612. 


\section{ZENO PARADOX: A RELATIVISTIC APPROACH TO SOLUTION}

Eureka

Volume 2, Number 1 (2011)

Matías A. O Quiroga

Grupo de Materiales y Sistemas Catalíticos, Departamento de Física, Universidad Nacional del Sur, Avda. Alem 1253, Bahía Blanca, B8000CPB, Argentina - CONICET

\section{Abstract}

Zeno of Elea was brilliant producing paradox [1]; the most famous is the story of Achilles and the tortoise. It can be summarized in these words: Achilles and the tortoise decide to have a race. Because Achilles can run twice as fast as the tortoise he gives her a long head start. Now, says Zeno, by the time Achilles reaches the tortoise's starting point she would have moved ahead by half the distance of her lead. And by the time Achilles reaches that point she would have moved on by half of that distance. And so on, and so forth, ad infinitum. Achilles is never able to catch up with the tortoise, because at each point, by the time he has covered the distance between them, she will always have moved on further by half of that distance. As Magee [1] points, it is here an impeccable logical argument that leads to a false conclusion. As Borges [2] reports, many previous works had focused looking for a fault in the logic [3] but they all have failed, so Borges suggests looking back to the concept of our world.

In this work, we shall use such suggestion focusing in the relativity theory. We are convinced that the inaccessibility of the paradox lies in work under the Galilean transforms in the Newtonian world. Despite this, we will try to see the problem under the Lorentzian transforms in the Eistenian world. For this purpose we first take a look at the paradox in Galilean mathematical terms and then we will look at the paradox under Lorentzian transform.

\section{Introduction}

The Zeno paradox has an interest, especially in quantum mechanics where had been already treated as a singular effect named the quantum Zeno effect $[4,5]$. In the present work, we will continue with the Malykin [6] discussion and also to present the paradox in maths words in order to shed light to it.

The Zeno paradox could be written in mathematical terms by the use of Galilean transforms. So we can write the all time position of Achilles like this:

$$
X_{A}=V_{A} t
$$

and the all time position of the tortoise:

$$
X_{T}=X_{0}+V_{A} t
$$

where $V_{A}$ and $V_{T}$ are the Achilles and tortoise respecting velocities, $X_{0}$ is the initial tortoise position and $t$ is the time. As in the introduction example, $V_{A}=2 V_{T}$, and also, must exist a time $t_{0}$ so that $V_{A} \cdot t_{0}=X_{0}$. Hence, $t_{0}=X_{0} / 2 V_{T^{*}}$. Then, with the help of easy operations, we can rewrite the equations (1) and (2) at the time that Achilles arrives to $X_{0}$.

$$
\text { Achilles: } \quad X_{A 1}=X_{0}=V_{A} t_{0}
$$

And the position of the tortoise at the same time:

$X_{T 1}=X_{0}+V_{T} t_{0}=X_{0}+\frac{V_{T} X_{0}}{2 V_{T}}=X_{0}+\frac{X_{0}}{2}$

Then Achilles needs a distance equal to $V_{A} \cdot t / 2=X_{0} / 2$ to reach to the tortoise. It means, that distance is $2 V_{T} \cdot t_{0} / 2$, hence $t_{0} / 2=X_{0} /\left(4 V_{T}\right)$
And the new positions will be:

Achilles:

$$
X_{A 2}=X_{0}+\frac{X_{0}}{2}
$$

And tortoise:

$X_{T 2}=X_{0}+\frac{X_{0}}{2}+V_{T} \frac{t_{0}}{2}=X_{0}+\frac{X_{0}}{2}+\frac{X_{0}}{4}$

And so on. If we carry on with this procedure we find two similar series. Already founded by Scottish mathematician James Gregory [7, 8], who has demonstrated and discussed the convergence of this series. From now on, we will name the Achilles position and the Tortoise position as $X_{A N}$ and $X_{T N}$, to illustrate those series behaviour.

$$
\begin{gathered}
X_{A N}=X_{0} \sum_{0}^{N}\left(\frac{1}{2}\right)^{N} \\
X_{T N}=X_{0} \sum_{0}^{N}\left(\frac{1}{2}\right)^{N}=X_{0} \sum_{0}^{N}\left(\frac{1}{2}\right)^{N+1}
\end{gathered}
$$

Mathematically, the paradox lies in asking both series to be equal for some $N$ value. Because of philosophical reasons, the $N$ number can not be infinite. To take this inappropriate number $(\infty)$ is to think that Achilles already reaches the tortoise and this is forbidden in the logical steps. It is clear, that for any $N$ number different from infinity, both series cannot be equal.

To make things easier we can rewrite the equations (3) and (4) as successions.

Remember that $\sum q^{M}=q\left(1-q^{M}\right) /(1-q)$, where $q$ is the reason term of the series. In this example $q=1 / 2$. 
We are asking to

$$
X_{0} \frac{\left[1-\left(\frac{1}{2}\right)^{N+1}\right]}{1-\left(\frac{1}{2}\right)} \text { be equal to } X_{0} \frac{\left[1-\left(\frac{1}{2}\right)^{N}\right]}{1-\left(\frac{1}{2}\right)}
$$

for some $N$ value.

This is clearly inconsistent. So we will take the Borges suggestion.

\section{Results and discussion}

In the Eistenian world we have to think again in the concept of simultaneity. What does it mean to be at the same time, at the same place? Both Achilles and the tortoise will not have any longer the same time, unless there exists an universal privileges clock. So, we must focus in only one of the subjects of our example. Let us think Achilles' situation under Lorentzian transform. The new first distance between Achilles and the tortoise is not just $X_{0}$, but $X_{0} / \gamma$. Where $\gamma$ is $\sqrt{1-\left[V_{A} / C\right]^{2}}$; where $C$ is the speed of light in the vacuum and Achilles' self time will be $t / \gamma$.

Hence, the hypothesis, the conditional equation (5) will turn to:

$$
\frac{X_{0}}{\gamma} \frac{\left[1-\left(\frac{1}{2}\right)^{N+1}\right]}{1-\left(\frac{1}{2}\right)}=X_{0} \frac{\left[1-\left(\frac{1}{2}\right)^{N}\right]}{1-\left(\frac{1}{2}\right)}
$$

Now, with the aid of the $\gamma$ term (which, in general, is very close to 1 for no relativistic movements) it cannot be said that it will not exist any $N$ value different from infinity which will allow thinking that equation (6) is true. From eq. (6) it is easy to find that we have to ask to the $N$ number to respect:

$$
\frac{\left[1-\left(\frac{1}{2}\right)^{N+1}\right]}{1-\left(\frac{1}{2}\right)^{N}}=\gamma
$$

Of course, the discussion is not finished. Despite we worked with an example, it is not hard to be more general with velocity relations. Even more, the paradox must be looked under other referencing systems or more complex theories. The Heisenberg uncertainty principle can give many views, taking into account that we are working with almost infinitesimal distances.

From the philosophical point of view, we think that this treatment can shed light on a full theory of the world. If the Zeno paradox cannot be resolved under a simple theory of the world (like the Greeks' or Newtonian), does it mean that the more complex theory, like relativity, is not just true but also necessary?

\section{Acknowledgment}

M.A.O Quiroga is knowledge with CONICET and Universidad Nacional del Sur for financial support.

\section{References}

[1] Bryan Magee, The Story of Philosophy, 1998 Dorling Kindersley Limited, London.

[2] Jorge Luis Borges. Obras Completas I,Discusión, 1996 Emecé Editores S. A. Buenos Aires.

[3] Bertrand Russel, Introduction to Matehmatical Philosophy, George Allen and Unwin 1919.

[4] B Misra and E. C. G Sudarshan 1977 J. Math. Phys. 18756.

[5] W. M. Itano, J. Phys Conference Series 196 (2009) 012018.

[6] G B Malykin, Phys, Uspekhi 45 (8) 907 (2002).

[7] Dictionary of Scientist, David Millar, Ian Millar, John Millar, Margaret Millar 1996, Cambridge University Press.

[8] De las Tortugas a las Estrellas, Leonardo Moledo, 1994 A.Z Editora S.A. 


\section{ORGANIC AMNESIA: A DIVERSITY IN DEFICITS}

Christopher R. Madan

Department of Psychology, University of Alberta

\section{Abstract}

Organic amnesia is the loss of memory due to biological factors such as brain disorders, tumors, strokes, degenerative diseases, or any other of a multitude of other disruptions of neurological function. Memories are stored throughout the brain, and as a result damage to any localized brain region only causes limited memory deficits. Even in itself, organic amnesia can present in a variety of impairments across numerous memory systems within the brain. Several kinds of amnesia will be reviewed, including details of the corresponding deficits and suggestions of the likely affected memory system.

\section{Introduction}

Many people believe that memory disorders affect memory as a whole. However, this is not the case as memory is not a unitary faculty of the mind (Tulving and Schacter, 1990). Memory disorders do not simply affect all of memory, rather they only impair select memory systems. Amnesia is commonly caused by memory loss due to specific circumstances, mainly brain injury, illness, or psychological trauma (Passer and Smith, 2007, p. 257). As described by Budson and Price (2005), "memory loss is often the most disabling feature of many disorders, impairing the normal daily activities of the patients and profoundly affecting the families." Classically, amnesia is when "a person has problems recalling information from the past" (DANA Foundation, 2007), however, there are many other possible memory impairments.

Amnesic syndromes also have found their place in Hollywood, albeit usually becoming distorted. In movies such as 50 First Dates (2004) and Groundhog Day (1990), the amnesic syndromes presented are impossible to replicate in the real world, and are often cacophony of real symptoms viewed from an overly simplistic standpoint. This is not surprising as popular culture tends to take a "mix and match" approach when it comes to portraying amnesic syndromes (Baxendale, 2004). Amnesia has also been demonstrated on big screen recently through many other movies ranging from The Bourne Identity (2002) trilogy to The Eternal Sunshine of the Spotless Mind (2004) and Memento (2000). The representation of amnesia, along with other neuropsychological disorders, in popular culture is quite important as it can have a significant effect on public perceptions of those who actually exhibit the disorder.

Amnesia can be divided into many different categories, two of which are based on the proximal cause of the impairment: organic amnesia and psychogenic amnesia. The biological instances of the amnesia, organic amnesia, is usually caused by brain disorders, tumours, strokes, degenerative diseases, chronic usages of select drugs, temporal lobe surgery, or electroconvulsive therapy (DANA Foundation, 2007). The psychological memory disorders, psychogenic amnesia, are much rarer occurrences than organic amnesia and are characterized by a loss or dissociation of one's identity and past experiences (Simons and Graham, 2000). As suggested by Fujiwara, Brand, Kracht, Kessler, Diebel, Netz, and Markowitsch (2008), some instances of retrograde psychogenic amnesia may actually be organic amnesia where the "functional/ metabolic disturbances in memory-relevant brain structures may have been below thresholds for detection". In some cases, the opposite may also be true, where the organic amnesia is entirely or in part caused by a psychological mechanism (Fujiwara et al., 2008).

In the literature, the best known case of organic amnesia is patient H.M., who, in the 1950s, had an operation to relieve symptoms of severe epilepsy (Scoville and Milner, 1957; Corkin, 2002). The surgery involved his hippocampal complex and parts of his temporal lobes in both hemispheres. After surgery, H.M. developed profound amnesic symptoms even though his epilepsy had improved (Simons and Graham, 2000). Since Milner and colleagues' initial research with H.M., many more cases of hippocampal amnesia have been researched (Spiers, Maguire, and Burgess, 2001). Although bilateral hippocampal lesions are clearly causing profound amnesia, there is still controversy regarding the extent that unilateral hippocampal lesions cause amnesia (Spiers et al., 2001). H.M. is now typically used as a benchmark case in the study of the role of the medial temporal lobe in learning and memory (O'Kane, Kensinger, and Corkin, 2001).

Some diseases, such as Alzheimer's, Parkinson's, and Huntington's disease, which are not commonly associated with amnesia, also encompass memory impairments. Due to their neurodegenerative nature their resulting lesions are progressive, rather than focal. For example, in medial temporal lobe damage: H.M. was a focal lesion patient. On the other hand, in Alzheimer's disease, a neurodegenerative disease, patients tend to experience medial temporal lobe damage first, while later other structures become involved as well (Budson and Price, 2005). Focal lesions are caused by specific incident, such as surgery or trauma. In contrast, progressive lesions are caused by neurodegenerative diseases that progress, affecting more of the brain and nervous system throughout their course.

Amnesia can vary greatly between individuals. This can be explained by attributing different memory systems to the various anatomical brain regions that have come to be associated with the amnesic syndrome. The many disorders that give rise to amnesia, "including herpes encephalitis, severe hypoxia, certain vascular lesions, head injury, deep midline tumours, basal forebrain lesions, and occasionally early dementia" (Kopelman, 2002), also are associated with differences in the resulting symptoms. Depending on the type of amnesia and the suspected cause, treatments vary greatly. For example, "one drug, donepezil, has been found to improve thinking and memory in patients with Alzheimer's disease" (DANA Foundation, 2007). In spite 


\section{R. Madan / Eureka 2 (2011)}

of this, impairments to the episodic memory system are much more prevalent than any other memory system impairments (Spiers et al., 2001). "Episodic memory is our personal, conscious, explicit recollection of experienced events" (Budson and Price, 2001).

Organic memory impairments can be subdivided further according to many different classifications, including time (as seen in anterograde and retrograde amnesias), processes (encoding, consolidation, storage, retrieval), and content (depending on the affected memory systems). Memory systems can range from unconscious, as in priming, to what is commonly thought of as memory, the episodic memory system. The aetiologies of memory impairing disorders can vary significantly and are quite diverse in their features and mechanisms of their resulting deficits.

\section{Forms of Amnesia}

As mentioned, amnesia can also be differentiated by its effect relative to the onset of its appearance. If the memory deficit presents as a severe inability to retain new information, it is classified as anterograde amnesia (Kapur, 1999). On the other hand, if the impairment presents as a loss of memory that had already been acquired prior to the onset of the cerebral pathology, it is considered retrograde amnesia.

Anterograde and retrograde amnesia often occur concurrently. The most common pattern is characterised by a temporal gradient followed by Ribot's Law (Shimamura, 1992). Ribot's Law illustrates that there is a particular pattern where the "ability to learn new information is most impaired (anterograde amnesia), recently learned information cannot be retrieved (retrograde amnesia), and remote learned information is spared" (Budson and Price, 2001). Budson and Price (2001) also mention that "the period of retrograde amnesia can last anything from several hours (in the case of transient global amnesia) to several years (in the case of surgical excision of the temporal lobes)." This is not to say that amnesia is always graded as advocated by Squire, Clark, and Knowlton (2001), ungraded retrograde amnesia can occur from damage to structures of the lateral and anterior temporal lobe, while graded amnesia is often associated with medial temporal lobe damage.

Anterograde amnesia is primarily caused by damage to the hippocampal complex, impairing the individual's ability to acquire new memories (Simons and Graham, 2000). As described by Simons and Graham (2000), retrograde amnesia is much less localized physiologically, though the temporal lobe appears to be a principal storage site for human memory. Most research into amnesia has been focused on anterograde amnesia as it is considered to be more disabling in everyday life, leaving the study of retrograde amnesia widely neglected by researchers and clinicians until recently (Kapur, 1999). Several reasons for this neglect, include that clinicians and caregivers alike are often less aware of retrograde memory symptoms and that there are significantly fewer retrograde memory tests available to clinical neuropsychologists, as opposed to anterograde memory tests.
Kopelman (2002) suggests that anterograde amnesia can result from possible disturbances in all memory processes: faulty encoding, faulty consolidation, accelerated forgetting, and faulty retrieval. Various studies point to each of the above causes, likely due to differing pathological conditions causing the resulting amnesic syndromes. Faulty encoding is when there is a deficit in the process responsible for the initial representation of the information, such as in Korsakoff patients. Faulty consolidation occurs when the impairment prevents the information from being effectively transferred from the initial representation to a relatively permanent store, a prominent occurrence head injury patients. However, when the information is learned adequately learned, problems may still cause the deficits in retention, as in individuals with Alzheimer's disease. Lastly, faulty retrieval may also occur, where the impairment exists independently of the information acquisition.

Most instances of memory impairment are associated with a temporally limited retrograde amnesia (Banich, 2004, p. 328). The DANA Foundation (2007) reports that these temporary amnesia cases are due to the disruption of normal brain function for brief periods of time, without causing permanent brain damage as seen in closed head injuries. The most common cause of this disruption is due to a blow to the head, from a sports injury or automobile accident, interfering with the consolidation process (DANA Foundation, 2007). Other longer, localized, disruptions can be observed in transient ischemic episodes where there is a loss of language skills (anomia) while still retaining recollection of the word-finding task (Kapur, 1999).

The memory consolidation period is believed to be approximately 20 to 30 minutes, "the time it takes your brain to file an input or experience away in long-term memory" (McConnell, 1989, p. 279). When the consolidation process is not functional, new memories fail to be stored into longterm memory, resulting in anterograde amnesia. This was observed in H.M. who "could not recall what he had for lunch and could not even recall if he had lunch at all" (Shimamura, 1992), and referred to his condition as always "waking from a dream". Pharmacological factors can also have profound effects on the memory consolidation process (Gold, 2006). Gold (2006) explicates that these factors can be demonstrated principally through manipulations of hormonal levels as well as through protein synthesis inhibitors.

As explained by Banich (2004, p. 336), amnesics have very specific patterns of limited memory loss, and despite amnesics losing their ability to remember information, under certain special circumstances, their behaviours are indicative of learning and memory. Amnesics can still learn, though not always in the traditional sense. One way of assessing residual memory in the presence of amnesia, is to utilize a content-based classification of memory or memory systems (Ashby and O'Brien, 2005). Traditionally, amnesia is associated with an inability to recall previous events, a property of the episodic memory system, but there are also several other memory systems that can be affected by neurodegenerative diseases. With such classification, one can observe and describe memory loss in some memory domain and preserved memory in others. 


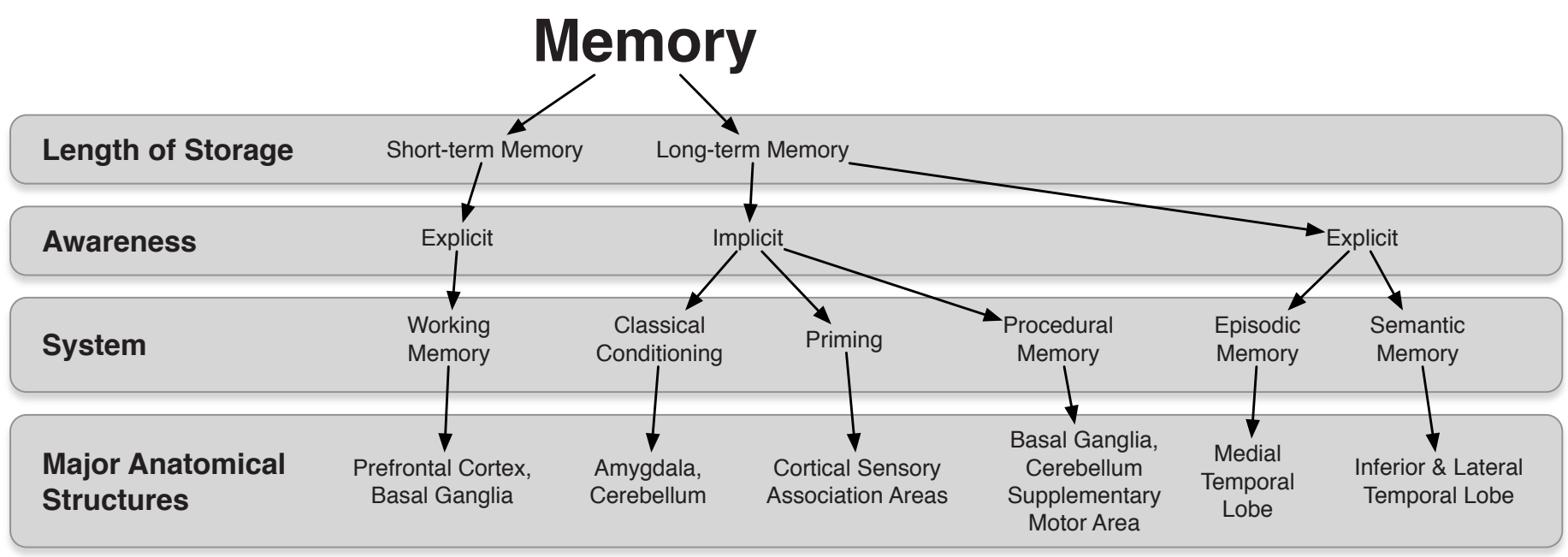

Figure 1. Memory System Classification. (Adapted from: Schacter and Tulving, 1994; Willingham, 1997; Budson and Price, 2001; Ashby and O’Brien, 2005)

\section{Memory Systems}

A 'memory system' is "a way in which the brain process information that is available for use at a later time, with or without conscious awareness" (Budson and Price, 2001). Of the various memory systems theorized, they can be categorized based on their length of storage and our conscious awareness of the memories in the system, and can be dissociated from the other systems (Schacter and Tulving, 1994). The length of storage can be either longterm or short-term memory, while our awareness is either explicit or implicit. Systems associated with conscious awareness are considered explicit, while others that are associated with behaviour changes or unconscious action are designated as implicit.

In Schacter and Tulving's (1994) memory system classification system, the only short-term memory system mentioned is working memory. However, the actual duration (and even existence) of short-term memory is debated by memory researchers. The conventional assumption is that short-term memory is limited to a few seconds and/or seven plus or minus two memory items (Banich, 2004, p. 239). Working memory is considered to be an explicit, declarative system as it requires "active conscious participation" (Ashby and O'Brien, 2005). Budson and Price (2001) give an example of "silently repeating a seven-digit phone number until you have a chance to write it down." Banich (2004, p. 239) affirms that the most common working memory task would be a digit-span task where items have to be reversed (i.e., repeating a sequence of numbers in reverse order). Ashby and O'Brien (2005) suggest that working memory is crucial for rule-based categorical learning, as assessed in the Wisconsin Card Sorting Test. Functional imaging studies suggest that working memory performance relies on the prefrontal cortex and basal ganglia (Ashby and O'Brien, 2005). Budson and Price (2005) list disorders such as Alzheimer's, Parkinson's, and Huntington's disease, as well as schizophrenia as having been shown to impair working memory, in addition to traumatic brain injuries. Attentional disorders including attention-deficit hyperactivity disorder, obsessive-compulsive disorder, schizophrenia, and depression can also impair working memory (Budson and Price, 2005).
When discussing long-term memory disorders, there are five memory systems theorized (Budson and Price, 2001). These systems are either implicit in nature, as in classical conditioning, priming, and procedural memory, or explicit, as in episodic and semantic memory.

Classical conditioning is the theory behind the traditional stimulus-response relationship, best observed through Pavlov's experiments (Budson and Price, 2001). Classical condition relies primarily on the amygdala and the cerebellum, for emotional and skeletal motor responses respectively (Willingham, 1997). Classically, amnesic patients are not impaired in conditioning tasks, though there are some exceptions. Budson and Price (2001) reveal that damage to this system can occur in progressive stages of Alzheimer's disease and in some Korsakoff syndrome (from mediodorsal thalamic nuclear damage) patients, as well as in cerebellar degenerative diseases. Conditioning a tone to be associated with a puff of air to the eye, causing the subject to blink, is a common test of classical conditioning (Shimamura, 2003). Amnesic subjects in this task often retain the eye blink response for up to 24 hours, though they did not recognize the testing apparatus.

Priming is another type of long-term, implicit, memory and is similar to the procedural and semantic memory systems (Tulving and Schacter, 1990). According to Willingham (1997), the basis for this system is that subjects are asked to study materials, then after a period of time, are given a task that utilizes the same study materials. Tulving and Schacter (1990) report that priming appears to be unaffected in most amnesic patients, neural degeneration associated with aging, and drug effects. Anatomically, priming is associated with the neocortical regions that mediate the priming sensory information, i.e. the occipital lobe for visual priming, though large lesions to the sensory association cortex can also cause deficits in priming (Budson and Price, 2001). Due to the implicit and covert nature of priming, impairments in this system are unlikely to be noticed outside of an experimental setting.

Last of the implicit memory systems is procedural memory, which "underlies changes in skillful performance and appropriate responding to stimuli" (Tulving and Schacter, 1990). A prime example of a procedural memory 


\section{R. Madan / Eureka 2 (2011)}

task is learning to ride a bike. Budson and Price (2005) report that functional neuroimaging has found procedural memory to be rooted in the basal ganglia, cerebellum, and the supplementary motor area. As a result, diseases that affect the basal ganglia, including Parkinson's disease and Huntington's disease, can impair this memory system (Budson and Price, 2001). Exclusive cerebellar damage, as found in some strokes and tumours, can also cause deficits to this memory system.

Many examples of procedural memory-based tasks have been used in research of the dissociations between memory systems. In these tasks amnesic patients are able to learn motor skills or be successfully primed without being able to recall having learned the tasks at all, though the use of explicit memory does greatly enhance an individual's ability to acquire procedural memory-based skills (Willingham, Nissen, and Bullemer, 1989). Corkin (2002) illustrates an example of this learning in experimental practices in H.M.'s improving performance over time in a mirror-tracing task (tracing the outline of a given shape, usually a 6-pointed star, within a narrow boundary while looking at the reflection of the page in the mirror). This type of learning has been further extended to involve mirror-reading (reading mirror-reversed text), though the amnesic patients were still impaired in recognizing the words (Willingham et al., 1989). In current neuropsychological assessments, the aforementioned tasks have been succeeded by the serial reaction time task (Ashby and O'Brien, 2005), involving the subject pressing keys as quickly as possible as a response to stimuli presented on a screen. In this task, the response time diminishes significantly when sequences are introduced, even when the subject is unaware that there is a sequence.

Despite the dissociation between procedural memory and the other memory systems, it can be more difficult to isolate procedural memory than is commonly stated. One example of this is demonstrated in Xu and Corkin's (2001) attempts to teach the H.M. to solve the Tower of Hanoi puzzle. In this study, even though H.M. did show some improvement throughout the day in the task under certain conditions, the learning was not carried over to the next day. Another example of this problem has been shown in studies with amnesics playing the computer game Tetris and then later having hypnagogic images of the game despite having no conscious recollection of having played the game at all. However, learning is improvished when compared with novice controls, suggesting a reliance of Tetris proficiency on declarative memory in addition to the procedural memory system (Stickgold, Malia, Maguire, Roddenberry, and O'Conner, 2000).

The remaining two memory systems are classified as explicit in nature, and are collectively referred to as declarative memory, although there is some debate between memory disorder researchers as to classification of these memory systems. Shimamura (2003) describes "memories that are tied to one's autobiography" as episodic memory, while semantic memory as "the vast database of factual knowledge stored in memory". These two memory systems also appear to be lateralized in the brain, as language processes are primarily located in the left cerebral hemisphere, while spatial processing is in the right hemisphere. Shimamura (2003) suggests that since episodic memory is largely spatial in nature, damage to the right hemisphere causes more deficits in this memory system than in the semantic memory system. In concordance, semantic memory impairments are often associated with lesions to the left cerebral hemisphere.

Deficits in episodic memory are the most common aspect of the amnesic syndrome. Squire and Zola (1998) describe episodic memory as "the capacity for recollecting happenings from the past, for remembering events that occurred in particular spatial and temporal contexts." Anatomically, episodic memory is based around the Papez circuit, originally proposed by Papez in 1937 (Budson and Price, 2001). This neuroanatomical circuit illustrates how information is transferred in and out of the hippocampus via the cingulate gyrus, in the medial temporal lobes. Squire et al. (2001) also report that "the hippocampus itself is important for memory for a relatively short period of time after learning and that the adjacent perirhinal and parahippocampal cortices remain important for a longer time." Some of the disorders that disrupt this memory system include: Alzheimer's disease, Korsakoff's syndrome, Herpes encephalitis, severe hypoxia, vascular disorders, transient global and epileptic amnesias, and head injuries (Kopelman, 2002). These syndromes are the primary causes of anterograde amnesia as well as some retrograde amnesias.

Budson and Price (2001) also notes that some aetiologies such as vascular and frontal lobe dementia are also known to cause distortions of episodic memory, as opposed to outright memory failure. In these cases, the origin of the deficit is not caused by damage to the Papez circuit, but rather damage in the frontal lobes. In the analogy proposed by Budson and Price (2001), the frontal lobes act as a 'file clerk', with the medial temporal lobes acting as the 'recent memory file cabinet' and other cortical regions serving as a 'remote memory file cabinet'. If the frontal lobe has been damaged, improper filing occurs and the memories may be distorted and inaccurate. Some forms of amnesia, caused by solely by frontal lobe pathology, are thus characterised mainly by faulty retrieval processes (i.e. head injury with exclusive frontal lobe damage) and memory distortions (Kopelman, 2002). In other diseases, such as Alzheimer's disease, both damage to the temporal lobe and frontal lobe regions co-occur with progression of the disease, causing genuine memory loss along with memory distortions.

Depending on the exact nature of the episodic memory deficits, it is possible to develop strategies to overcome the impairments. Huppert and Piercy (1978) report that patients with Korsakoff's syndrome were able to achieve picture recognition performance comparable to the control subjects after observing the stimulus for 4-8 times longer than the controls. They suggest the extra time allowed the Korsakoff patients to achieve the same level of learning that the controls reached in less time. The deficits found on the patients only affect the initial learning and not the subsequent recall (Huppert and Piercy, 1978). However, Squire and Zola (1998) suggest that the mechanism for this process may rely on the semantic memory system. 


\section{R. Madan / Eureka 2 (2011)}

The last memory system, semantic memory, is based in understanding concepts and general knowlege. Studies of semantic memory have localized it to the inferolateral temporal lobe and the memory system is commonly impaired in Alzheimer's disease and semantic dementia patients (Budson and Price, 2005). In the progression of Alzheimer's disease, the most vulnerable and first affected system appears to be episodic memory and then, in later stages, also semantic memory. (Kensinger, Ullman, and Corkin, 2001). Tulving and Markowitsch (1998) believe this is because the episodic memory system is a special case of semantic memory. Still, not all memory researchers agree with this view (Squire and Zola, 1998).

Semantic memory impairments can be seen in the inability to name items that were previously well-known, as well as impoverished general knowledge (Budson and Price, 2001). In cases with mild semantic memory deficits, difficulties in naming items of a certain category may occur, as seen in propagnosia, where the patient's ability to recognize faces is impaired (Shimamura, 2003). In propagnosia, brain damage is limited to the fusiform gyrus, which is specialized for facial recognition. These patients can still recognize familiar individuals by their voice. Other mild impairments may involve inabilities in naming animals or tools (Budson and Price, 2001). As outlined by Kopelman (2002) there is still, there is some dispute as to the actual mechanisms behind some of the dissociations found between different categories.

Semantic memory is also believed to be partially dependent on the medial temporal lobe, commonly damaged in amnesics experiencing episodic memory deficits (Squire and Zola, 1998). This view is supported by studies showing that episodic and semantic memory alike are impaired in patients with hippocampal damage as both memory systems share many commonalities (Tulving and Markowitsch, 1998). However, Squire and Zola (1998) state that in patients with frontal lobe damage, deficits in episodic memory are exhibited while semantic memory appears to be intact. Even still, semantic memory does appear to be partially dissociable from the hippocampus, as seen in studies of H.M. (Kensinger et al., 2001). In studies of H.M. it appears that episodic memory aids in the initial learning of semantic information, but is no longer needed for the retrieval, relying on neocortical regions rather than the hippocampus.

Similarly to episodic memory, even with semantic impairments, it is possible to gain and acquire new information over prolonged periods of time. H.M. was able to draw an accurate floor plan of his home that he moved to after the onset of his amnesia (Corkin, 2002). O'Kane et al. (2004) also report that H.M. was able to learn new famous names via slow-learning and extended repetitions. These findings suggest that less effective, alternative mechanisms for semantic memory-based learning can still occur after brain damage has occurred.

In further analyzing the declarative memory systems, through recognition tasks a particularly intriguing instance has arisen -- familiarity. These tasks are based on the 'remember-know procedure' designed to differentiate between recollecting (through retrieval of episodic details) the word presented and feelings of familiarity of the word (Quamme, Yonelinas, and Norman, 2007). While tests of recollection-based recognition require the hippocampus, familiarity judgements appear to be spared in cases of hippocampal amnesia (Spiers et al., 2001). Stark and Squire (2000) ruled out priming as a mechanism for this effect in their study. Quamme et al. (2007) instead propose that limited medial-temporal lobe amnesia can be associated with spared capacity for familiarity discriminations. Quamme et al.'s (2007) study also was able to mimic the effect found in hippocampal amnesics with undergraduate students instructed to use a specific familiarity-based strategy. Research into this mechanism is still just beginning and will be an key step in memory research differentiating the two declarative memory systems.

Though several of these memory systems are not commonly associated with the amnesic syndrome, such as classical conditioning deficits, their resulting symptoms can be classified as amnesic in nature (Budson and Price, 2001). As stated by Willingham (1997), other researchers have proposed further breakdowns to the previously described memory systems. The deficits presented by the various memory system impediments found in diseases and syndromes associated with organic amnesia can be quite diverse and it is unlikely for an individual to experience impairments in multiple memory systems.

\section{Conclusion}

McConnell (1989, p. 278) describes the effect of amnesia as "the process by which [information] is physically erased from your memory banks, blocked off from easy access, or prevented from being stored in the first place." Even more importantly, as described by Corkin (2002), "amnesia is not an all-or-nothing condition," though Budson and Price (2001) mention that some diseases such as Alzheimer's can impair multiple memory systems. In real-life, most situations rely on multiple memory systems (Ashby and O'Brien, 2005).

Organic amnesias are memory disturbances that are caused by specific malfunctions in the brain (DANA Foundation, 2007). Shimamura (2003) mentions that the location of the brain lesion is not the most important feature, but rather the size of the lesion, that determines the severity of the impairment. Memories are stored throughout the brain, and as a result damage to any localized brain region only causes limited memory deficits. However, a large brain lesion would lead to greater and more profound memory impairments. The precise deficits found in individuals with amnesia can vary a great deal, although there are some commonalties, such as the "severe loss of post- and pre-morbid episodic memories in virtually all patients with bilateral hippocampal damage" (Spiers et al., 2001). As mentioned by Spiers et al. (2001), other features may vary, such as the semantic memory and familiarity-based recognition.

Though there is a great diversity in disorders involving organic amnesia symptoms and there are several memory systems that can be affected, often the resulting impairments can be diminished through the use of compensatory strategies. 


\section{R. Madan / Eureka 2 (2011)}

\section{References}

Ashby, F.G and O'Brien, J.B. (2005). Category learning and multiple memory systems. TRENDS in Cognitive Sciences, 9(2), 83-89.

Banich, M.T. (2004). Cognitive Neuroscience and Neuropsychology (2nd ed.). Boston, MA: Houghton Mifflin.

Baxendale, S. (2004). Memories aren't made of this: amnesia the movies. British Medical Journal, 329, 1480-1483.

Budson, A.E. and Price, B.H. (2001) Memory: Clinical Disorders. Encyclopedia of Life Sciences. Chichester: John Wiley and Sons, Ltd.

Budson, A.E. and Price, B.H. (2005). Memory Dysfunction. New England Journal of Medicine, 352, 692-699.

Corkin, S. (2002). What's new with the amnesic patient H.M.? Nature Reviews Neuroscience, 3, 153-160.

DANA Foundation. (2007). Amnesias. The DANA Guide to Brain Health. Retrieved October 17, 2007, from http://www.dana.org/brainhealth/ detail. aspx?id=4716.

Fujiwara, E., Brand, M., Kracht, L., Kessler, J., Diebel, A., Netz, J., Markowitsch, H.J. (2008). Functional retrograde amnesia: a multiple case study. Cortex, 44, 29-45.

Gold, P.E. (2006). The many faces of amnesia. Learning and Memory, 13, 506-514.

Huppert, F.A and Piercy, M. (1978). Dissociation between learning and remembering in organic amnesia. Nature, 275, 317-318.

Kapur, N. (1999). Syndromes of retrograde amnesia: A conceptual and empirical synthesis. Psychological Bulletin, 125(6), 800-825.

Kensinger, E.A., Ullman, M.T., and Corkin, S. (2001). Bilateral medial temporal lobe damage does not affect lexical or grammatical processing: Evidence from amnesic Patient H.M. Hippocampus, 11, 347-360.

Kopelman, M.D. (2002). Disorders of memory. Brain, 125, 2152-2190.

McConnell, J.V. (1989). Understanding Human Behavior (6th ed.). New York: Holt, Rinehart and Winston.

O'Kane, G., Kensinger, E.A., Corkin, S. (2004). Evidence for semantic learning in profound amnesia: An investigation with Patient H.M. Hippocampus, 14, 417-425.

Passer, M.W., and Smith, R.E. (2007). Psychology: The Science of Mind and Behavior (3rd Ed.). New York: McGraw-Hill.
Quamme, J.R., Yonelinas, A.P., Norman, K.A. (2007). Effect of unitization on associative recognition in amnesia. Hippocampus, 17, 192-200.

Schacter, D.L. and Tulving, E. (1994). Memory Systems. Cambridge, MA: MIT Press.

Scoville, W.M. and Milner, B. (1957). Loss of recent memory after bilateral hippocampal lesions. Journal of Neurology, Neurosurgery and Psychiatry, 20, 11-21.

Shimamura, A.P. (1992). Organic Amnesia, from L.R. Squire (Ed.), Encyclopedia of Learning and Memory, pp. 30-35. New York: Macmillan.

Shimamura, A.P. (2003). Neural basis of memory: Systems level. In L. Nadel (Ed.), Encyclopedia of Cognitive Science, London: Macmillan.

Simons, J.S. and Graham, K.S. (2000). Amnesia, from G. Fink (Ed.), Encyclopedia of Stress, pp. 176-178. San Diego: Academic Press.

Stark, C.E. and Squire, L.R. (2000). Recognition memory and familiarity judgements in severe amnesia: No evidence for a contribution of repetition priming. Behavioural Neuroscience, 114(3), 459-467.

Stickgold, R., Malia, R., Maguire, D., Roddenberry, D., and O'Conner, M. (2002). Replaying the game: Hypnagogic images in normals and amnesics. Science, 290, 350-353.

Spiers, H.J., Maguire, E.A., and Burgess, N. (2001). Hippocampal Amnesia. Neurocase, 7, 357-382.

Squire, L.R., Clark, R.E., and Knowlton, B.J. (2001) Retrograde Amnesia. Hippocampus, 11, 50-55.

Squire, L.R. and Zola, S.M. (1998). Episodic Memory, Semantic Memory, and Amnesia. Hippocampus, 8, 205-211.

Tulving, E. and Markowitsch, H.J. (1998). Episodic and declarative memory: Role of the hippocampus. Hippocampus, 8, 198-204.

Tulving, E. and Schacter, D.L. (1990). Priming and human memory systems. Science, 247, 301-306.

Willingham, D.B., Nissen, M.J., and Bullemer, P. (1989). On the development of procedural knowledge. Journal of Experimental Psychology: Learning, Memory, and Cognition, 15(6), 1047-1060.

Willingham, D.B. (1997). Systems of memory in the human brain. Neuron, 18, 5-8.

Xu, Y. and Corkin, S. (2001). H.M. revisits the Tower of Hanoi puzzle. Neuropsychology, 15(1), 69-79. 


\section{THE BIGGEST BUGS: AN INVESTIGATION INTO THE FACTORS CONTROLLING THE MAXIMUM SIZE OF INSECTS

Delyle Polet

Department of Biological Sciences, University of Alberta

\section{Introduction}

Modern insects are much smaller than their prehistoric counterparts. The largest extant insect is three times smaller in mass than the heaviest of ancient insects. Whether there are indeed factors that have changed over the 400 million years of insect evolution leading to their smaller size and whether there is a single limiting factor are unsolved questions. In entomology, these two subjects are met with numerous conjectures; a proper analysis of them all would require a lengthy treatise. For the sake of brevity, this review looks at some of the most familiar suspects: the insect tracheal system, changes in atmospheric oxygen content over earth history and the evolution of flight. Other perhaps less common theories such as the changes in ecology in insect habitats, the insect exoskeleton, and the evolutionary advantage of being small are also topics of discussion.

In this paper, "largeness" and "smallness" will refer to mass as opposed to length, width or volume. However, one cannot often determine mass from insect fossils since some fossils only contain wings or other pieces of an insect's body. In some articles that have been cited here, only the body length is reported. In these cases, one can only assume that a larger wingspan or longer body means a more massive insect. When the comparison of size switches to wing length, or length, I will make that distinction. I will discuss only adult insects, even though the larval stages of a given species are commonly larger than the adult stage.

\section{Oxygen and Protodonata}

The largest modern insect recorded for its weight was a "Giant Weta", or Deinacrida heteracantha, at 71g. Even in this case, it was an exception: a pregnant female with an exceptionally large brood (Williams 2001). 300 million years ago, the Meganeura monyi roamed the skies (Atkinson 2005). It had a $71 \mathrm{~cm}$ wingspan, with a body $30 \mathrm{~cm}$ long and $3 \mathrm{~cm}$ wide (Maina 2005). Assuming its body was roughly cylindrical and had a density about equal to that of water, it weighed in approximately at $210 \mathrm{~g}$. This mass would make it the largest insect known in history (Atkinson 2005).

A common theory circulating in paleontology is that the massive oxygen changes over the past 500 million years of earth's history have contributed to change in insect size (Butterfield 2009; Okajima 2008). Insects distribute oxygen to their cells through a tracheal system. This system

Note: This article was originally prepared as part of the University of Alberta's Science 100 program.

For more information about Science100, see:

http://www.science.ualberta.ca/ProspectiveStudents/SCIENCE100.aspx depends on diffusion to a greater extent than other oxygen distribution systems, such as our own circulatory system. For diffusion of oxygen, the higher the concentration of oxygen in the atmosphere, relative to the inside of the organism (the concentration gradient $\Delta \mathrm{C}$ ), the faster the rate of diffusion of that oxygen (Fick's First Law):

$\mathrm{dm} / \mathrm{dt}=\mathrm{DS} \Delta \mathrm{C} / \mathrm{x}$

$D$ is the diffusion coefficient, $m$ is mass, $S$ is surface area, $\mathrm{x}$ is distance, and $\mathrm{t}$ is time. This one-dimensional equation assumes that the concentration gradient doesn't change throughout the diffusive distance, but is a good approximation of many biological systems (Vogel 2003).

A simple approximation shows how diffusion can limit insect size. As the length (I) of an insect doubles, the surface area of its body increases by $l^{2}$. If the surface area of the spiracles, or tracheal openings, is proportional to the surface area of the insect, then these openings also increase by $1^{2}$. By Fick's law, then, rate of diffusion increases by 2 . However, since volume and therefore mass scale approximately proportionally to $I^{3}$, the insect is fully eight times more massive. The new tissue now needs more oxygen. The metabolism of an insect scales approximately with surface area, $I^{2}$ (Alexander 2002), so has increased by 4. The diffusive rate of oxygen cannot keep up with the new energy needs.

A large insect like Meganeura sp. would need faster diffusion to make up for its great size, assuming that it also had a tracheal system. Since a higher amount of atmospheric oxygen means a larger concentration gradient, it follows that the $\sim 30 \%$ atmospheric oxygen in the Carboniferous period, the time of Meganeura, would have allowed for faster diffusion, and thus, larger insects (see Fig 1).

The theory is further substantiated by the often-large size of decapods (e.g. lobsters and crabs). Members of this arthropod order have an oxygenated circulatory system and can reach considerably larger sizes than insects (SchmidtNielsen 1997). The largest land crab, the coconut crab (Birgus latro), can reach sizes of $4 \mathrm{~kg}$ - almost 20 times larger than the largest insect in history (Terrestrial Ecoregions 2010). Despite having a similar body plan to insects in their segmented body and chitin-based exoskeleton, decapods can reach much larger sizes (Elzinga 2004). Since a key difference between insects and decapods is the tracheal system, perhaps the oxygenated circulatory system is the limiting factor of maximum size in insects.

However, there is a suite of major problems with this hypothesis. First of all, the mathematical model relating insect wingspan to atmospheric oxygen does not always correlate with one another, as a study by Okajima showed 


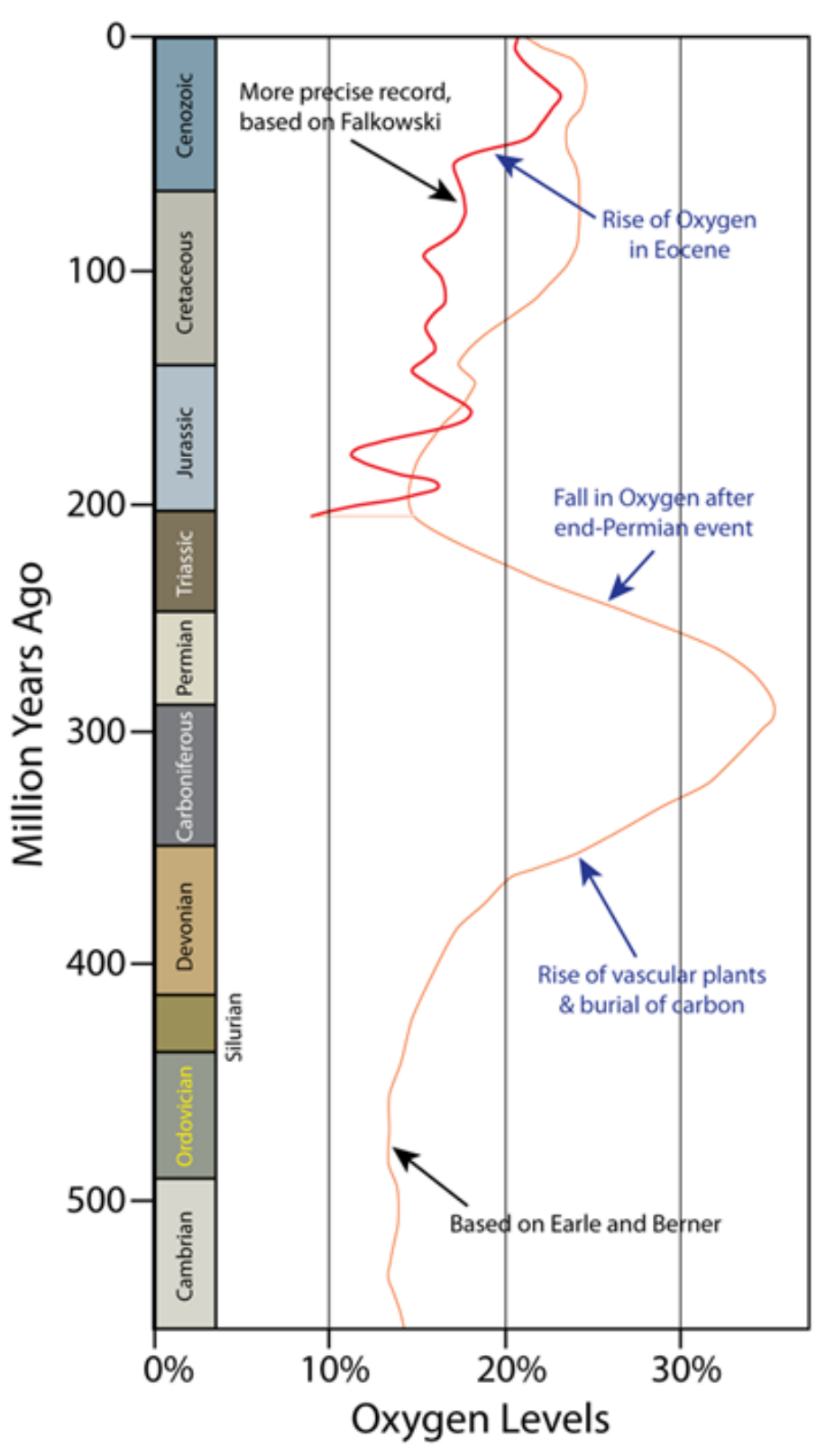

Figure 1. Atmospheric Oxygen Levels through geological time [source:essayweb.net/geology/timeline/phanerozoic.shtml]

in 2008. For example, dragonfly fossils from the Triassic and Jurassic show an insect with a wingspan double that of the largest dragonfly today, but atmospheric oxygen levels were one-half to three-quarters of modern levels. Secondly, modern insects have ways to compensate for low oxygen levels. A study by Loudon (1989) showed that when modern dragonflies were reared in different oxygen levels, the cross sectional area of their trachea increased with lower oxygen levels. Since, by Fick's law, mass of oxygen over time depends also on surface area, the increased surface area of the dragonflies' trachea compensates for the lower concentration gradient. Furthermore, diffusion is not the only tool of distributing oxygen in insect tracheal systems. Weis-Fogh (1964) analyzed the tracheal system in the flight muscles of insects. His conclusion was that in order to allow for passive diffusion to account for the metabolic needs in flight, the maximum diameter of a dragonfly's thorax should be approximately $0.5 \mathrm{~cm}$. Because thoraxes exceeding
$0.5 \mathrm{~cm}$ exist in modern dragonflies, he attributed these excesses to non-diffusive mechanisms. A study by Miller (1966) showed that a thoracic pump in beetles contributes to a large portion of their oxygen demand in flight. Similarly, thoracic pumps are believed to contribute heavily to the in-flight oxygen demand of dragonflies, moths, and many other insects (Weis-Fogh 1964; Miller 1966).

There are other ways through which insects can overcome diffusion, which are only recently being uncovered. Many species of insects have been shown to exhibit discontinuous gas exchange, where they close their spiracles for extended periods of time before opening them again. The "fluttering phase" of this process uses the slightly negative air pressure built up from oxygen use to suck in fresh oxygen. The process is thought to transport oxygen faster than diffusion (Nation 2008). The insect tracheal system is still poorly understood and more study may yet reveal processes not yet considered. Finally, some have argued that high oxygen levels would favour smaller insects, if any size at all, since smaller flying animals require a faster metabolism relative to their body size compared with large animals (Butterfield 2009).

Although atmospheric oxygen levels are not the only factor controlling insect size, they are still an important factor to consider. The insect tracheal system depends on diffusion to a greater extent than other systems and may still constrain insect size somewhat but certainly not by the amount supposed through pure diffusive processes (Dudley 2000; Weis-Fogh 1964). Currently, knowledge of past oxygen levels is the subject of much debate, and a better grasp on how atmospheric oxygen has changed may still shed some light on why insect size has changed (Butterfield 2009). However, oxygen levels are certainly not the only factor.

\section{Exoskeleton}

The exoskeleton has also played a key role in insect development and diversity. Their hard shells saves them from dry conditions on land and can protect them from predators (Elzinga 2004). For a class of terrestrial animals that started small, an exoskeleton would be a wise choice.

Many scientists have studied whether the tracheal system limits insect size, but the exoskeleton - another key component of insect physiology - seems to have been mostly overlooked as a constraint to size. The biggest land animals in history all had an internal skeleton made of bone, such as a Mammoth or Supersaurus, a large dinosaur. Whether an exoskeleton made of Chitin that prevents insects from becoming large is an issue worth considering.

A study by Currey (1967) compared the endoskeleton to the exoskeleton, based purely on mechanical properties. He treated the legs like upright columns and applied formulae for buckling, rupture, compression, bending, and impact loading. The exoskeleton, he concluded, is the better choice for static loading, the kind of loading one's legs experience when one is standing still. The endoskeleton, with its surrounding soft tissue, is much better for dynamic loading, which is mostly experienced through activity, like running and jumping. Smaller organisms will experience less dynamic loading due to their lower momentum at 
normal activity speeds (Vogel 2003). Currey concluded that the endoskeleton is more advantageous for large organisms and the exoskeleton for smaller organisms. The insects' dependence on an exoskeleton makes them ideal for small sizes, but as they get bigger the exoskeleton is a burden. The opposite is true of tetrapods: their endoskeleton makes them adaptable to being large, but it keeps them from achieving the size of the smallest arthropods.

The constraint on the exoskeleton by itself cannot explain why the largest modern insects are so much smaller than the largest ancient insects. We don't know much of how the exoskeleton has changed over time. However, assuming that the materials of the endo- and exoskeletons have remained relatively constant over earth's history, one can come to an interesting hypothesis when one also consider the changes in ecology between the Carboniferous and now.

\section{Ecology}

Atmospheric oxygen increased in the Jurassic and the wingspan of the Ordonata became smaller over the same time period. There was one major problem with models comparing atmospheric oxygen concentration to insect size. Okajima (2008) pointed out that the emergence of flying vertebrates could explain this particular case of shrinking. In the Paleozoic, insects were the only animals in the skies. However, Pterosaurs showed up in the Triassic and birds in the Jurrasic, competing with the insects for aerial space. Pterosaurs and birds can get much larger than insects, possibly due to their endoskeleton, hollow bones and oxygenated blood. The factors make them a more likely predator than the insect. A big insect is a more obvious prey for something much bigger. To adapt, insects became smaller.

Modern studies on butterfly larvae have agreed with the theory. In a laboratory setting, large caterpillars were selected preferentially by a Picromerus bidens predator (Berger and others 2006). In the experiment, two species of Lepidoptera were used. The results show that it did not matter which species were looked at or what colour they were. Larvae at the same size saw the same risk of predation, with larger larvae having a larger risk.

The largest of endoskeletal animals were all flightless varieties. One should think that the same would be true of insects. Indeed, Giant Wetas are flightless and the largest of modern insects. Uninhibited by the need to fly, one may question whether flightless insects in the Carboniferous can easily outgrow the flight-endowed species. On the ground, insects never reached the $30 \mathrm{~cm}$ length that their flying cousins managed. In fact, the largest Paleozoic flightless insect found so far in the literature is a $6 \mathrm{~cm}$ long silverfish called Ramsdelepidion schusteri. Perhaps instead of granting them girth, their inability to fly doomed them to a life of miniature.

While insects in the air mostly had to worry about each other, insects on the ground had to compete with giant tetrapods. Like birds and pterosaurs, the endoskeleton and oxygenated respiratory system allows earth-bound tetrapods to get much larger than insects. Therefore, tetrapods became the major predators. As expected from the model, land-dwelling insects adapted to these new competitors by downsizing. Predation has helped to push both winged and wingless insects to the size one sees today.

Since predation pushes animals to become smaller, one may ask why can terrestrial crabs are so large. The coconut crab may be a case of island gigantism since they develop on isolated islands where no large terrestrial predators had been established (Gan and others 2008; Brockie and others 1988). Still, the answer in this case may indeed remind one of the oxygenated blood in these crustaceans and perhaps even in the harder Chitin and higher number of supporting legs in these animals.

The Giant Weta may also be a case of this sort of "island gigantism". They are endemic to New Zealand, an island country that has no native land mammals besides bats (Mclntyre 2001). Because Giant Wetas are nocturnal, provide food for owls and distribute seeds much like small rodents in other ecosystems, Giant Wetas are the ecological analogue of mice in New Zealand (Mclntyre 2001). This is a clear example of how the changes in ecosystem have affected insect size. Due to the lack of competitors in the niche occupied by mice, insects grew into that niche by evolution and thus began growing to sizes comparable to those of mice. When mice were introduced to New Zealand, the Giant Weta population declined drastically (Mclntyre 2001). The same may be generally true of insects over their evolutionary history. Since the physiology of tetrapods makes them more adaptive to those niches occupied by large organisms, they out-competed the large insects. Because insects are more adaptive to niches occupied by small organisms, they better adapt to those niches.

\section{The Evolutionary Advantage of Small Size}

In terms of animal species diversity, insects are the undisputed champions. Estimates for insect diversity range from as much as 80 million species to as few as 5 million species. Even at the lowest estimate, insects represent around half of global species diversity (Gullan and Cranston 1994). A major reason for this success is the fact that they are small. Gullan and Cranston (1994) convey a very good illustration for the phenomenon. Consider a single acacia tree. One tree can provide one meal for a giraffe but can also support the complete life cycles of dozens of insects. As an extreme example, a single tree surveyed in Uganda hosted 37 species of ants alone (Shulz and Wagner 2002).

The smallness of insects allows them to fill niches left vacant by larger organisms. It also may have helped them survive four major mass extinctions and survive on land (McKinney 1997). Warm-blooded species such as mammals and birds have increased difficulty at small sizes because they have to maintain an incredibly high metabolism (Alexander 2002). As previously mentioned, even cold-blooded tetrapods are at a serious disadvantage at small sizes because of their endoskeletons. The insects filled ecological vacuums that other organisms simply could not fill (Gullan and Cranston 2005). Evolution would have selected for those insects that kept their small size. This does not fully explain why there are no insects larger than $70 \mathrm{~g}$ today, only that evolution makes such sizes less likely. 
Indeed, there is some degree of overlap: large beetles can exceed the sizes of small mice, bats and reptiles.

\section{Conclusion}

The issue of why ancient insects could be so big and modern ones are not is an area of fierce debate with many viewpoints. The tracheal system in insects is a likely contender but by how much is still under scrutiny. Their exoskeletons make them well suited to small sizes but not as much to larger sizes. Their ability to fly, in combination with their choice of skeleton, may have reduced their size somewhat. All these factors contributed to initially reduced sizes in comparison to endoskeletal competitors with oxygenated blood. Over time, insects evolved to occupy smaller niches where their endoskeletal competitors were at a disadvantage.

Not a single one of the above theories can by themselves explain the modern maximal insect size. By taking into account the limitations of insect respiration, skeleton and predation within the larger context of evolution, we've come up with a plausible explanation for the range of insect sizes discussed in this paper. A more detailed analysis of all the largest insects over time, along with any dramatic changes in the above factors contributing to their size, is required before any general conclusions can be made. An even more developed answer would take into account other factors, for example, geography, sexual selection, climate, and nutrition.

The small sizes of insects have enormously contributed to their success. It allows them to have faster reproduction times, enabling them to adapt quickly to change, and allows them to survive times of trouble when food is scarce. Perhaps the question we should be asking is not "why are insects small" but rather "why be big?" At over 5 million species, and having dominated terra firma longer than any other animals, insects seem to be doing quite nicely on this planet. Ironically, these little creatures are the true titans of planet Earth.

\section{References}

Alexander DE. 2002. Nature's flyers: birds, insects, and the biomechanics of flight. Baltimore: Johns Hopkins University Press. p. 358.

Atkinson TP. 2005. Arthropod body fossils from the union chapel mine. In Buta, R J, Rindsberg, A K and Kopaska-Merkel D C, editors. Pennsylvanian footprints in the Black Warrior basin of Alabama: Alabama Paleontological Society, monograph, no. 1. Burmingham, AL: Alabama Paleontological Society, p. 169-176.

Berger D, Walters R, Gotthard K. 2006. What keeps insects small? Size dependent predation on two species of butterfly larvae. Evol. Ecol. 20:575-589.

Blanckenhorn W. 2000. The evolution of body size: What keeps organisms small? Q. Rev. Biol. 75(4):385-407.

Borrell BJ. 2004. Mechanical properties of calcified exoskeleton from the neotropical millipede, nyssodesmus python. J. Insect Physiol. 50(12):1121-6.

Butterfield NJ. 2009. Oxygen, animals and oceanic ventilation: An alternative view. Geobiology 7:1-7.
Candy DJ. 1981. Hormonal regulation of substrate transport and metabolism. In: Downer RGH (ed.). Energy metabolism in insects. p. 19.

Chown SL, Gaston KJ. 2009. Body size variation in insects: a macroecological perspective. Biol. Rev. 85:139-69.

Currey JD. 1967. The failure of exoskeletons and endoskeletons. J. Morph. 123:1-16.

Dudley R. 2000. The biomechanics of insect flight: Form, function, evolution. Princeton University Press, Princeton, NJ (USA). p. 476.

[EEC] Earth's Endangered Creatures. c2010. Queen Alexandra's Birdwing Butterfly Facts. [Internet; cited 2010 4/11/2010]. Available from: http://www.earthsendangered.com/profile. $a s p$ ? view=all\&ID=4\&sp=52.

Elzinga RJ. 2004. Fundamentals of Entomology (6 ed). Upper Saddle River, NJ: Pearson. p. 495.

Gan C, Tee S, Tang P, Yang JM, Freire F, McGowan A, Narriman $\mathrm{J}$, Mohammed MS, Hsieh $\mathrm{H}$, Chen $\mathrm{C}$, et al. 2008. Isolation and characteristics of 10 microsatellite markers from the endangered coconut crab (Birgus latro). Molecular Ecology Resources 8:14481450.

Gillott, C. 2005. Entomology (3 ed). Saskatoon, SK: Springer. p.831.

Gullan PJ, Cranston PS. 1994. The Insects: An Outline of Entomology. London: Chapman and Hall. p. 491.

Gullan PJ, Cranston PS. 2005. The Insects: An Outline of Entomology (3 ed.). Oxford: Blackwell Publishing. p. 528.

Heinrich B. 1993. The hot-blooded insects: Strategies and mechanisms of thermoregulation. Cambridge MA: Harvard University Press.

Heinrich B. 1995. Insect thermoregulation. Endeavour 19(1):28-33.

Maina JN. 2005. Comparative respiratory morphology. Heidelberg, Germany: Springer. p. 172.

Mclntyre M. 2001. The ecology of some large Weta species in New Zealand. In: Field LH (ed.). The Biology of Wetas, King Crickets and their Allies. Wallingford, UK: CABI Publishing. p. 225-239.

McKinney ML. 1997. Extinction Vulnerability and selectivity: combining ecological and paleontological views. Annu. Rev. Ecol. Syst. 28:495516.

Nation JL. 2008. Insect physiology and biochemistry (2 ed). Boca Raton, FL: CRC Press. p. 401-404.

Okajima R. 2008. The controlling factors limiting maximum body size of insects. Lethaia 41(4):423-40.

Parker J, Johnston LA. 2006. The proximate determinants of insect size. Journal of Biology 5: 15.1-15.4.

Schmidt-Nielsen K. 1997. Animal physiology: Adaptation and environment. Cambridge: Cambridge University Press. p. 120.

Shulz A, Wagner T. 2002. Influence of forest type and tree species on canopy ants (Hymenoptera: Formicidae) in Budongo Forest, Uganda. Oecologia 133(2):224-232.

Strongest Animal [Internet; cited 2010 4/3/2010]. Available from: http://www.extremescience.com/zoom/index.php/creepy-crawlies/117strongest-animal.

Terrestrial Ecoregions: Maldives-Lakshadweep-Chagos Archipelago tropical moist forests (IM0125) [Internet; cited 2010 4/2/2010]. Available from: http://www.nationalgeographic.com/wildworld/profiles/ terrestrial/im/im0125.html.

Tsutsui ND, Suarez AV, Spagna JC, Johnston S. 2008. The evolution of genome size in ants. BMC Evolutionary Biology 8:64.

Weis-Fogh T. 1964. Diffusion in insect wing muscle, the most active tissue known. J. Exp. Biol. 41:229-256.

Williams DM. 2001. Largest. In: University of Florida book of insect records. [Internet; cited 9 April 2010] Available from:

http://entnemdept.ufl.edu/walker/ufbir/chapters/chapter_30.shtml.

Vogel S. 2003. Comparative biomechanics. New Jersey: Princeton University Press. p. 580. 


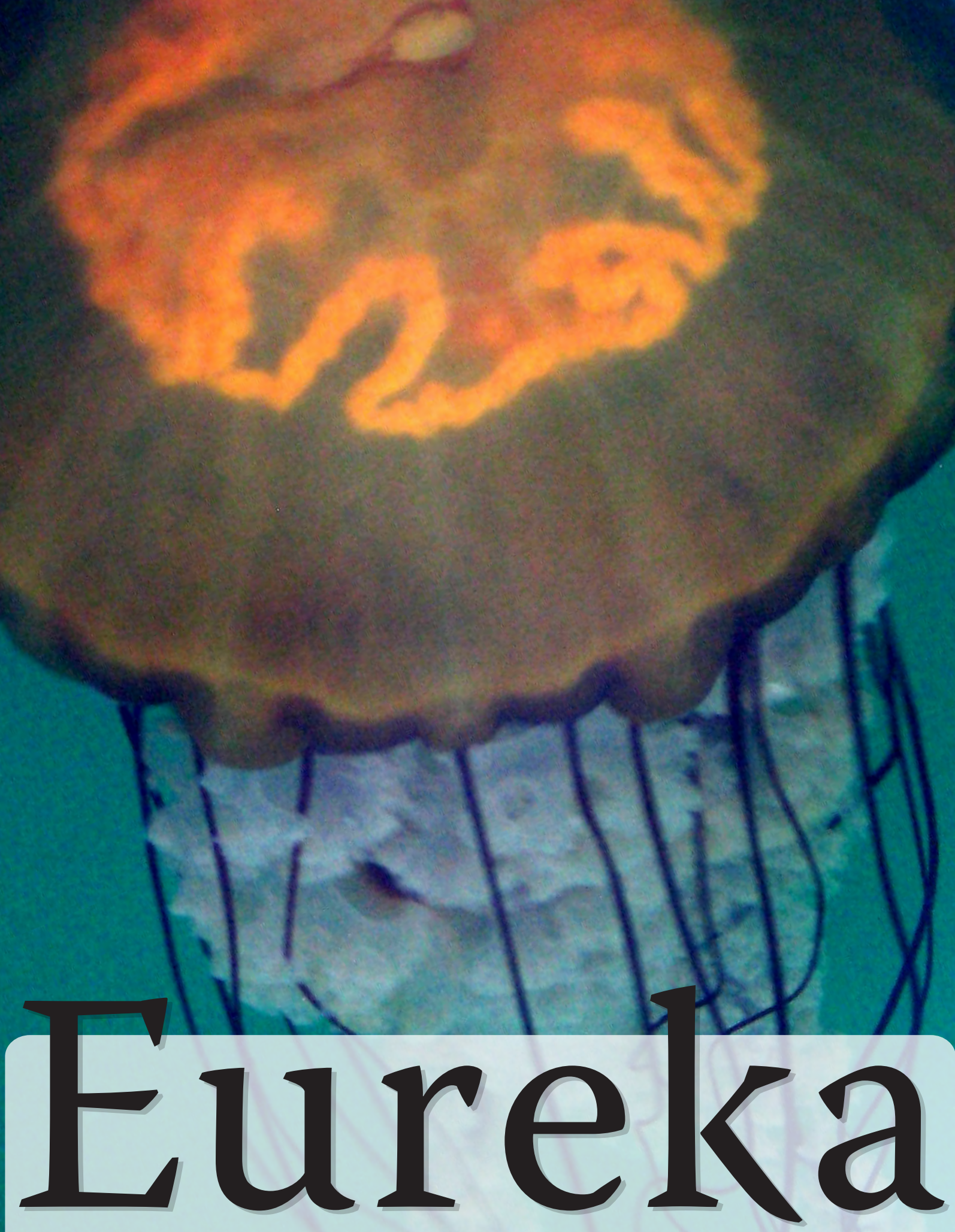

University of Alberta Science Undergraduate Research Journal http://www.eurekajournal.com

Volume 2, Number 1

April 2011 